\title{
Exploiting Polarimetric TerraSAR-X Data for Sea Clutter Characterization
}

\author{
Eduardo Makhoul, Member, IEEE, Carlos López-Martínez, Senior Member, IEEE, and \\ Antoni Broquetas, Member, IEEE
}

\begin{abstract}
5 Abstract-This paper presents a detailed characterization of 6 sea/ocean clutter returns at X-band, imaged by TerraSAR-X 7 (TSX) mission from the radiometric, statistical, and polarimet8 ric standpoints. Different TSX data takes, covering the typical 9 spaceborne incidence angle region $\left(20^{\circ}-\mathbf{4 5}^{\circ}\right)$, are analyzed: dual10 polarized and experimental quad-polarized (quad-pol) data have 11 been used. The thermal noise of the receiver for quad-pol data 12 turns out to be an important limitation in the sea characterization 13 from TSX, particularly at high incidence angles (above $36^{\circ}$ ), where 14 low signal-to-noise ratios (SNRs) can impair the proper data 15 distribution fitting and the polarimetric backscattering descrip16 tion. Statistical analysis shows large deviation from Gaussianity, 17 indicating presence of texture, mainly in the small incidence region $18\left(\mathbf{2 0}^{\circ}-\mathbf{3 6}^{\circ}\right)$, with favorable SNR conditions. The different polari19 metric features revealed contribution of nonpolarized scattering, 20 related to the presence of breaking waves. This paper also proposes 21 and evaluates an extension of the well-known X-Bragg model, 22 named X2-Bragg (extended-extended Bragg), which properly ac23 counts for the impact of thermal noise and sea clutter temporal 24 decorrelation, due to the dual receive antenna acquisition mode. 25 Such additional decorrelation sources, if not properly analyzed 26 and accounted for in the physical-based model description, could 27 lead to an incorrect interpretation of the polarimetric properties 28 and the related erroneous geophysical parametric inversion from 29 the real data. In this sense, the X2-Bragg proves its fitting to 30 the experimental data, quantifying accordingly the presence of 31 nonpolarized scattering in terms of the roughness parameter $\boldsymbol{\beta}_{1}$.
\end{abstract}

32 Index Terms-Non-Gaussian clutter, parameter estimation, 33 radar backscattering coefficient, sea clutter, statistical analysis, 34 synthetic aperture radar (SAR), SAR polarimetry, X-Bragg.

\section{INTRODUCTION}

${ }_{36} 7$ HE imaging capability of synthetic aperture radars (SARs), 38 independent from daylight and weather conditions, repre39 sents a potential tool for globally monitoring the ocean. SAR is 40 a very important forecast instrument in oceanography, allowing, 41 among others, the retrieval of ocean wave spectrums [1] and

Manuscript received April 24, 2015; revised July 9, 2015; accepted July 12, 2015. This work was supported in part by the FPU Research Fellowship Program, Ministerio de Educación under Contract AP2009-4590; by the FI-AGAUR Research Fellowship Program, Generalitat de Catalunya under Contract 2010FI EM051757; and by the Spanish Ministry of Science and Innovation (MICINN) under projects TEC2011-28201-C02-01 and TIN201455413-C2-1-P.

E. Makhoul was with the Department of Signal Theory and Communications, Universitat Politècnica de Catalunya, 08034 Barcelona, Spain. He is now with isardSAT, 08042 Barcelona, Spain (e-mail: edumak@tsc.upc.edu).

C. López-Martínez and A. Broquetas are with the Department of Signal Theory and Communications, Universitat Politècnica de Catalunya, 08034 Barcelona, Spain (e-mail: carlos.lopez@tsc.upc.edu; broquetas@tsc.upc.edu).

Color versions of one or more of the figures in this paper are available online at http://ieeexplore.ieee.org.

Digital Object Identifier 10.1109/TGRS.2015.2457242 the measurement of ocean or river current velocities [2]. Lately, 42 the increase in vessel hijacking, as well as the need to control 43 fishing and tanker polluting activities, demand remote sensing 44 systems to properly monitor maritime traffic. SAR sensors with 45 ground moving target indication (GMTI) capabilities are of 46 great interest for ship surveillance [3].

Characterization and modeling of the sea clutter returns 48 observed by SAR systems are of great interest for the accurate 49 evaluation of future SAR missions imaging the sea. The avail- 50 ability of polarimetric SAR instruments, such as TerraSAR-X 51 (TSX) or TanDEM-X (TDX), provides a unique opportunity to 52 gather geophysical and biophysical information of the marine 53 environment. This paper exploits different dual-pol $\left(\mathrm{HH} / \mathrm{HV}^{1}\right) 54$ and experimental quad-pol TSX data sets (covering $20^{\circ}-45^{\circ} 55$ of incidence angle) to perform a transversal (radiometric, sta- 56 tistical, and polarimetric) and compact study of the sea echo 57 returns. To the authors' knowledge, such analysis has not 58 been performed yet, exploiting the experimental quad-pol data 59 from TSX.

The main stress of the study is to point out the different 61 limitations that may be encountered when operating TSX, par- 62 ticularly in the quad-pol mode, where the dual receive antenna 63 (DRA) mode is used [4], such that the antenna is split into two 64 halves (receiving $\mathrm{H}$ and $\mathrm{V}$ simultaneously) and the transmit 65 polarization is toggled in a pulse basis. On one hand, there is 66 an increase of $3 \mathrm{~dB}$ in the noise equivalent sigma zero (NESZ) 67 due to a reduced antenna effective area per channel, i.e., 3-dB 68 loss in signal-to-noise ratio (SNR). On the other hand, a tem- 69 poral lag exists between the different polarimetric channels, 70 during which sea clutter decorrelates. The identification of such 71 technological-driven aspects from the signal-based analysis 72 proves to be of key importance in the correct interpretation of 73 the polarimetric data when fitting physical-based models. In 74 this sense, the X-Bragg model [5], which extends the Bragg 75 surface scattering to include the impact of depolarized and 76 cross-polarized returns, has been optimized to account for such 77 additional perturbations (noise+sea temporal decorrelations), 78 based on a comprehensive theoretical formulation, leading to 79 the extended-extended Bragg (X2-Bragg) model; otherwise, 80 these additional decorrelation sources could produce an erro- 81 neous interpretation of the polarimetric backscattering proper- 82 ties when trying to fit the physical-based X-Bragg model to the 83 experimental data.

The radiometric analysis, in terms of the radar backscattering 85 coefficient $\sigma^{0}$, shows that, in TSX, the thermal noise of the re- 86

${ }^{1} \mathrm{H}$ and $\mathrm{V}$ refer to horizontal and vertical polarizations, respectively. 
TABLE I

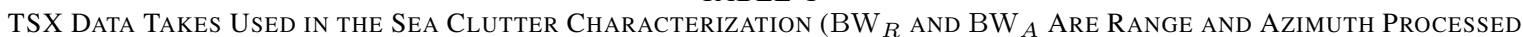
BANDWIDTHS, $v_{\text {win }}$ AND $\theta_{\text {win }}$ ARE Wind SPEED AND Direction From Which THE Wind Is Blowing)

\begin{tabular}{|c|c|c|c|c|c|c|c|c|c|}
\hline ID & Date & Region & Location & Mode & $\gamma[\mathbf{d e g}]$ & $\boldsymbol{B W}_{\boldsymbol{R}}[\mathbf{M H z}]$ & $\boldsymbol{B W}_{\boldsymbol{A}}[\mathbf{H z}]$ & $\boldsymbol{v}_{\boldsymbol{w i n}}[\mathbf{m} / \mathbf{s}]$ & $\boldsymbol{\theta}_{\boldsymbol{w i n}}[\mathrm{deg}]$ \\
\hline \hline TSX-1 & 05.03 .2013 & Senegal & N15.07-W17.60 & Duad-Pol & $19.85-21.74$ & 150 & 1380 & - & - \\
\hline TSX-2 & 09.03 .2013 & Gulf of Guinea & N3.56-W2.35 & Duad-Pol & $22.43-24.26$ & 150 & 1380 & - & - \\
\hline TSX-3 & 25.04 .2010 & Vancouver Island & N49.72-W127.97 & Quad-Pol & $23.75-25.49$ & 150 & 1380 & 12.1 & 143 \\
\hline TSX-4 & 06.05 .2010 & Vancouver Island & N49.72-W127.97 & Quad-Pol & $23.75-25.49$ & 150 & 1380 & 6.3 & 331 \\
\hline TSX-5 & 16.03 .2013 & Mauritania & N19.45-W16.78 & HH & $24.80-27.99$ & 150 & 2765 & - & - \\
\hline TSX-6 & 30.04 .2010 & Bermuda & N31.37-W69.54 & Quad-Pol & $29.70-31.30$ & 150 & 1380 & 6.5 & 258 \\
\hline TSX-7 & 11.05 .2010 & Bermuda & N31.37-W69.54 & Quad-Pol & $29.70-31.30$ & 150 & 1380 & 6.9 & 62 \\
\hline TSX-8 & 05.03 .2013 & Mauritania & N18.78-W17.43 & Dual-Pol & $30.81-32.33$ & 150 & 1380 & - & - \\
\hline TSX-9 & 05.03 .2013 & Senegal & N13.91-W18.48 & Dual-Pol & $31.91-33.42$ & 150 & 1380 & - & - \\
\hline TSX-10 & 12.04 .2010 & Barcelona & N41.23-E2.11 & Quad-Pol & $33.04-34.50$ & 150 & 1380 & - & - \\
\hline TSX-11 & 28.04 .2010 & Southeast Yakutat & N58.20-W137.96 & Quad-Pol & $36.18-37.54$ & 150 & 1380 & 7.2 & 164 \\
\hline TSX-12 & 09.05 .2010 & Southeast Yakutat & N58.20-W137.96 & Quad-Pol & $36.18-37.54$ & 150 & 1380 & 5.7 & 350 \\
\hline TSX-13 & 23.04 .2010 & Pratas Island & N20.67-E116.92 & Quad-Pol & $39.03-40.29$ & 150 & 1380 & - & - \\
\hline TSX-14 & 25.04 .2010 & South Hecate Strait & N52.37-W129.81 & Quad-Pol & $40.02-41.25$ & 150 & 1380 & 13.1 & 140 \\
\hline TSX-15 & 06.05 .2010 & South Hecate Strait & N52.37-W129.81 & Quad-Pol & $40.02-41.25$ & 150 & 1380 & 6.7 & 318 \\
\hline TSX-16 & 17.03 .2013 & Gulf of Guinea & N0.24-E7.97 & Dual-Pol & $43.45-44.58$ & 150 & 1380 & - & - \\
\hline TSX-17 & 21.04 .2010 & North Sea & N54.38-E7.05 & Quad-Pol & $44.37-45.48$ & 150 & 1380 & - & - \\
\hline
\end{tabular}

87 ceiver could be an important limiting factor for proper sea clut88 ter characterization (statistical and polarimetric backscattering 89 fitting), particularly in the case of quad-polarized acquisitions 90 and for the spaceborne high-incidence region $\left(36^{\circ}-45^{\circ}\right)$ due 91 to the reduced SNR conditions. The $\sigma^{0}$ model, i.e., XMOD2 92 [6], optimized from TSX/TDX dual-pol data, provides a close 93 fitting to the measured data also for the quad-pol case, where 94 remaining calibration issues on the experimental DRA mode 95 may induce additional errors [7].

96 The statistical analysis based on the computation of the 97 normalized intensity moments (NIM) [8], and complemented 98 by the log-cumulants' study [9], [10] confirms deviation from 99 Gaussian statistics for the high-resolution X-band system [8], 100 [10]-[13], and mainly at incidence angles below $36^{\circ}$, where 101 sufficient SNR is available.

102 To evaluate the scattering properties of the sea when imaged 103 by TSX, the polarimetric roll-invariant parameters, i.e., entropy $104 H$ and mean scattering angle $\bar{\alpha}$ described in [14], as well 105 as the polarization ratio (PR) metrics [10], [15], have been 106 exploited. The results indicate that most of the acquisitions lie 107 in the low to medium random surface scattering region, mainly 108 due to the important contribution of nonpolarized returns, as 109 indicated by $H / \bar{\alpha}$ and PR analyses. The proposed X2-Bragg 110 model shows a good fitting with the experimental data in the $111 H / \bar{\alpha}$ plane, providing a mechanism to model the nonpolarized 112 returns in terms of $\beta_{1}$ parameter, which is an indicator of the 113 surface roughness (breaking waves events are known to cause 114 an increase in surface roughness [8], [16]).

115 The remainder of this paper is organized as follows. In 116 Section II, the TSX data set is presented. The methodology fol117 lowed to perform the sea clutter characterization is introduced 118 in Section III. In Section IV, the radiometric analysis in terms 119 of radar backscattering coefficient is presented. Later on, the 120 statistical description of the experimental data set is analyzed 121 in Section V. To end up the transversal characterization, a com122 plete polarimetric analysis is carried out in Section VI. Conclu123 sions are drawn in Section VII. A comprehensive derivation of 124 the proposed X2-Bragg model and its theoretical evaluation are 125 considered in Appendixes A and B.

\section{DATA SET DESCRIPTION}

The main parameters of the stripmap products used in the 127 analysis are summarized in Table I. The different data sets are 128 made up of acquisitions from both the TSX and TDX instru- 129 ments. Different aspects were considered for the selection of 130 the data under analysis: 1) covering the spaceborne SAR oper- 131 ational range of incidence angles from $20^{\circ}$ to $45^{\circ} ; 2$ ) avoiding 132 heterogeneity in the reflectivity over the imaged area; 3) avoid- 133 ing regions with high density of maritime traffic and/or marine 134 littorals (because of the high ambiguities' impact in the DRA 135 acquisition); and 4) when possible, polarimetric acquisition is 136 preferred over single polarization.

The available fully polarimetric TSX data were acquired 138 during the experimental DRA mode campaign, April and May 139 2010. It is well known that sea or ocean conditions, and hence 140 their radar response, could be quite different depending on the 141 geographical location of the marine scenario under analysis; 142 this is why it has been tried to sample the acquisitions over dif- 143 ferent oceans (Atlantic and Pacific) and seas (North, Mediter- 144 ranean, and China). For the nominal dual-polarization stripmap 145 products, the absolute and relative radiometric accuracies are 146 0.6 and $0.3 \mathrm{~dB}$, respectively; whereas for the quad-polarization 147 products, there is no specification, since it is an experimental 148 mode with few dedicated campaigns and additional investiga- 149 tions on the DRA calibration issues need to be considered [7]. 150

Portions of the images of three representative data takes 151 (near/TSX-4, middle/TSX-7, and far range/TSX-17) are shown 152 in Fig. 1; only copolarized channels $\left(S_{\mathrm{HH}}\right.$ and $\left.S_{\mathrm{VV}}\right)$ are consid- 153 ered since the cross-polar returns are corrupted by the thermal 154 noise. Data takes TSX-4, TSX-7, and TSX-17 correspond, 155 respectively, to high-, medium-, and low-SNR scenarios, with 156 mean values of $16.3,10.9$, and $3.7 \mathrm{~dB}$ for the copolar $S_{\mathrm{HH}} 157$ channel. The average SNR estimated values are summarized in 158 Table II for the different data takes, where the cross-polarized 159 returns have low SNR (most of them around 1-5 dB). Data 160 takes TSX-11 and beyond (incidence angle above $36^{\circ}$ ) have a 161 higher impact of the thermal noise, as indicated by the reduced 162 SNR conditions, even for the copolar channels. For TSX-4 163 [see Fig. 1(a) and (b)], the local surface wind produces an 164 


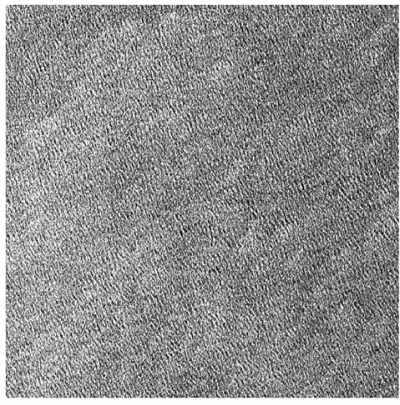

(a)

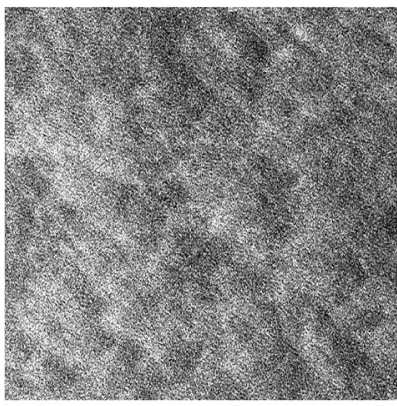

(c)

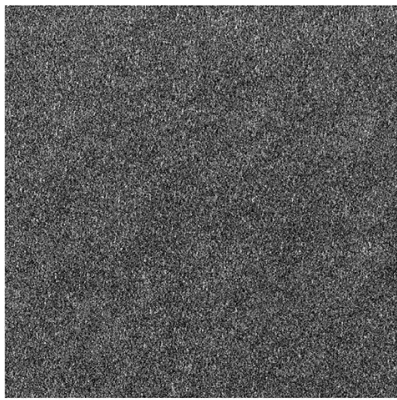

(e)

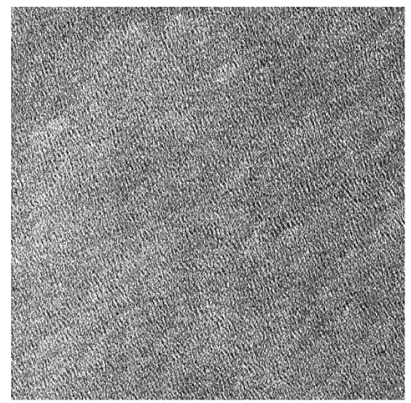

(b)

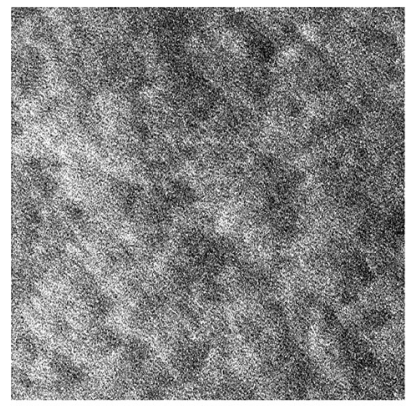

(d)

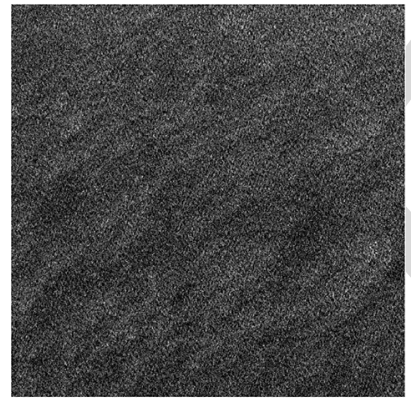

(f)
Fig. 1. Multilook $(11 \times 11)$ patches $\left(512 \times 512\right.$ pixels). (a) and (b) $S_{\mathrm{HH}}$ and $S_{\mathrm{VV}}$ for TSX-4 $\left(v_{\mathrm{win}}=6.3 \mathrm{~m} / \mathrm{s}\right.$ and $\left.\theta_{\mathrm{win}}=331^{\circ}\right)$. (c) and (d) $S_{\mathrm{HH}}$ and $S_{\mathrm{VV}}$ for TSX-7 $\left(v_{\text {win }}=6.9 \mathrm{~m} / \mathrm{s}\right.$ and $\left.\theta_{\text {win }}=62^{\circ}\right)$. (e) and (f) $S_{\mathrm{HH}}$ and $S_{\mathrm{VV}}$ for TSX-17 (no wind information).

TABLE II

Estimated SNR Average Values For the TSX Data TAKES

\begin{tabular}{|c|c|c|c|c|c|}
\hline \multirow{2}{*}{ ID } & \multirow{2}{*}[\mathbf{deg}]{} & \multicolumn{4}{|c|}{ SNR [dB] } \\
\cline { 3 - 6 } & & $\boldsymbol{S}_{\boldsymbol{H} \boldsymbol{H}}$ & $\boldsymbol{S}_{\boldsymbol{V} \boldsymbol{V}}$ & $\boldsymbol{S}_{\boldsymbol{H} \boldsymbol{V}}$ & $\boldsymbol{S}_{\boldsymbol{V} \boldsymbol{H}}$ \\
\hline TSX-1 & $19.85-21.74$ & 23.0 & - & 4.8 & - \\
\hline TSX-2 & $22.43-24.26$ & 19.6 & - & 4.3 & - \\
\hline TSX-3 & $23.75-25.49$ & 17.7 & 17.9 & 6.8 & 6.9 \\
\hline TSX-4 & $23.75-25.49$ & 16.3 & 16.5 & 5.9 & 6.1 \\
\hline TSX-5 & $24.80-27.99$ & 11.7 & - & - & - \\
\hline TSX-6 & $29.70-31.30$ & 8.7 & 9.9 & 3.8 & 4.0 \\
\hline TSX-7 & $29.70-31.30$ & 10.9 & 11.6 & 4.2 & 4.3 \\
\hline TSX-8 & $30.81-32.33$ & 9.2 & - & 4.0 & - \\
\hline TSX-9 & $31.91-33.42$ & 9.1 & - & 4.1 & - \\
\hline TSX-10 & $33.04-34.50$ & 6.5 & 8.6 & 0.9 & 1.3 \\
\hline TSX-11 & $36.18-37.54$ & 5.1 & 6.6 & 2.6 & 2.6 \\
\hline TSX-12 & $36.18-37.54$ & 5.3 & 6.2 & 3.2 & 3.3 \\
\hline TSX-13 & $39.03-40.29$ & 6.4 & 8.9 & 2.8 & 3.4 \\
\hline TSX-14 & $40.02-41.25$ & 5.8 & 8.8 & 2.6 & 3.4 \\
\hline TSX-15 & $40.02-41.25$ & 3.5 & 4.5 & 2.5 & 2.4 \\
\hline TSX-16 & $43.45-44.58$ & 2.8 & - & 2.2 & - \\
\hline TSX-17 & $44.37-45.48$ & 3.7 & 6.2 & 2.3 & 2.4 \\
\hline
\end{tabular}

165 appreciable wave pattern. For TSX-7 [see Fig. 1(c) and (d)], a 166 more turbulent scenario is observed with a front propagating 167 mainly in the diagonal direction. Turbulence, such as eddies or upwellings, could produce such response, bringing also 168 some organic material (surfactants) to the surface [17]. At the 169 highest incidence angle region, i.e., TSX-17, the HH channel 170 has a noise-like pattern [see Fig. 1(e)] compared with VV [see 171 Fig. 1(f)], where a wave pattern is roughly appreciated.

Ground truth data from the historical database of the National 173 Data Buoy Center of the National Oceanic and Atmospheric 174 Administration and the Canadian Moored Bouy of the DFO are $175 \quad \mathbf{A Q 2}$ available only for some acquisitions, as indicated in Table I. 176 The geographical location of the employed buoys matches the 177 center of the acquired images, but the temporal sampling rate 178 is not sufficient to determine the exact sea conditions. Typical 179 differences on time are between 30 and $60 \mathrm{~min}$ before or after 180 the acquisition time; however, in most cases, sea conditions are 181 quite stable in a 4-h window around the acquisition time.

\section{Sea Clutter Characterization Methodology}

183

A software module has been implemented to automatically 184 perform a complete and exhaustive characterization of the 185 data based on the three-level approach (radar backscattering 186 coefficient, ${ }^{2}$ statistics, and polarimetry). This analysis module 187 can accept as input a set of different data takes from different 188 sensors, where each is processed sequentially.

The first submodule performs a radiometric calibration, 190 based on the input product information as described in [18], 191 such that the intensity of the pixels at its output is related to $\sigma^{0} 192$ values. ${ }^{3}$ In the next block, the area of interest (AOI) is selected, 193 which is, to date, carried out simply by a visual inspection of 194 the image product considering a rectangular AOI. Afterward, a 195 subregion characterization based on a sliding window over this 196 very AOI is performed. It is possible to define the size of such a 197 sliding window and the overlapping in both range and azimuth 198 dimensions.

For the statistical characterization and the normalized reflec- 200 tivity $\sigma^{0}$ estimation, a window considering the whole azimuth 201 strip for each range bin (range line basis) within the AOI has 202 been selected. Therefore, the dependence of different descrip- 203 tors with the incidence angle can be studied. For polarimet- 204 ric decomposition, a rectangular sliding window of $11 \times 11205$ without overlapping has been used to avoid mixing different 206 scattering mechanisms and obtain adequate speckle filtering 207 [19]. As proposed in [5], an additive noise filtering (NF) (for 208 quad-pol data) can be performed in order to reduce the noise 209 impact. Then, the polarimetric description is performed before 210 and after NF.

Prior to sub-AOI dependent characterization, specific filter- 212 ing on the data can be optionally completed, ${ }^{4}$ such that the 213 impact of man-made structures present in the open sea (such as 214 vessels, oil platforms, and wind farms) is minimized, avoiding 215

\footnotetext{
${ }^{2}$ In the literature, the radar backscattering coefficient is also referred as normalized radar cross section (NRCS).

${ }^{3}$ It must be noted that those pixels with negative intensities, once the calibration is performed, are excluded from the subsequent characterization.

${ }^{4}$ This filtering method has been considered only for those data takes where a high density of high-reflectivity man-made targets is expected, based on a visual prescreening approach, taking into account the a priori geographical location of the region being analyzed; otherwise, and depending on the filtering threshold, the sea clutter data characterization could be impaired.
} 
216 corruption of the data analysis. A simple filter approach has 217 been implemented: those pixels, whose magnitude $y_{i}$

$$
y_{i} \geq \mu_{\text {subAOI }}+\beta \sigma_{\text {subAOI }}
$$

218 exceeds the mean statistical value $\mu_{\text {subAOI }}$ in a given number 219 of times $\beta$ the standard deviation $\sigma_{\text {subAOI }}$, are discarded. Both 220 statistical moments are estimated from the available samples 221 within the sub-AOI, whereas the $\beta$ term is experimentally 222 adjusted, with typical values between 2 and 6 .

\section{IV. RADIOMETRIC ANALYSIS (NRCS)}

224 Most of the available semiempirical models of $\sigma^{0}$ (NRCS) 225 are optimized for the low grazing region, based on ground226 based radar observations. Therefore, they are not applicable for 227 spaceborne SAR acquisitions typically at $20^{\circ}-45^{\circ}$ of incidence 228 angle. Recently, two models have been proposed: the first 229 one, the Naval Research Laboratory (NRL) [20], is based on 230 the Nathanson reference tables in [21], covering the region 231 of $30^{\circ}-90^{\circ}$; the second one, named XMOD2 [6], has been 232 optimized from TSX/TDX copolar data, for incidence angles 233 from $20^{\circ}$ to $45^{\circ}$. XMOD2 provides $\sigma^{0}$ values at $\mathrm{VV}$, and thus, $234 \mathrm{PR}$ models should be used to retrieve the HH component. In [6], 235 three models (T-PR, E-PR, and X-PR) have been adjusted for 236 copolarized TSX data.

237 The estimated $\sigma^{0}$ for the different data takes is depicted as 238 a function of the incidence angle in Fig. 2 for the copolar 239 channels. A sliding window in a range line basis is used, i.e., $240 \sigma^{0}$ is averaged using all the azimuth samples in a range bin. The 241 sea return decreases as a function of incidence angle, where the $242 S_{\mathrm{VV}}$ channel has, in general, a higher backscattering than $S_{\mathrm{HH}}$. 243 Cross-polar channels, not reported due to space limitations, 244 have low $\sigma^{0}$ values, close to noise level. NESZ is also reported 245 for the different data takes as dotted black lines in Fig. 2, 246 which can help in understanding the impact of the thermal noise 247 on each data take and thus the related SNR metric (estimated 248 average values are reported in Table II). Comparing the NESZ 249 of quad-pol data with dual-pol acquisitions (e.g., TSX-6 versus 250 TSX-8), a degradation around $3 \mathrm{~dB}$ is observed due to the 251 DRA mode operation. Therefore, the increase on NESZ (or 252 decrease in SNR) for quad-pol data will impair the proper 253 characterization of the sea clutter using TSX data. For some 254 data takes (e.g., TSX-11, TSX-12, TSX-15, and TSX-16), and 255 due to their reduced SNR conditions $(\approx 2-5 \mathrm{~dB})$, the effect of 256 the antenna elevation pattern at the edges of the corresponding 257 subswath can be clearly appreciated.

258 In Fig. 2, the range of copolar values predicted by the 259 NRL and XMOD2 models for a sea surface wind speed range 260 of $0.1-20 \mathrm{~m} / \mathrm{s}$ is indicated by the shaded regions, delimited 261 by dash-dot-dot and dashed gray lines, respectively. In the 262 XMOD2 case, an average of the upwind, crosswind, and down263 wind conditions has been considered. Moreover, the expected 264 values for $\mathrm{HH}$ have been also averaged for the different PR 265 models (T-PR, E-PR, and X-PR). For data takes with ground 266 truth information, the NRL model has an average deviation 267 w.r.t. the estimated values of 6.92 and $5.11 \mathrm{~dB}$ for $S_{\mathrm{VV}}$ and $S_{\mathrm{HH}}$ 268 channels, respectively; whereas XMOD2 presents a mean error 269 of $0.98 \mathrm{~dB}$ for $S_{\mathrm{VV}}$; and for the $S_{\mathrm{HH}}$ channel, the average de-

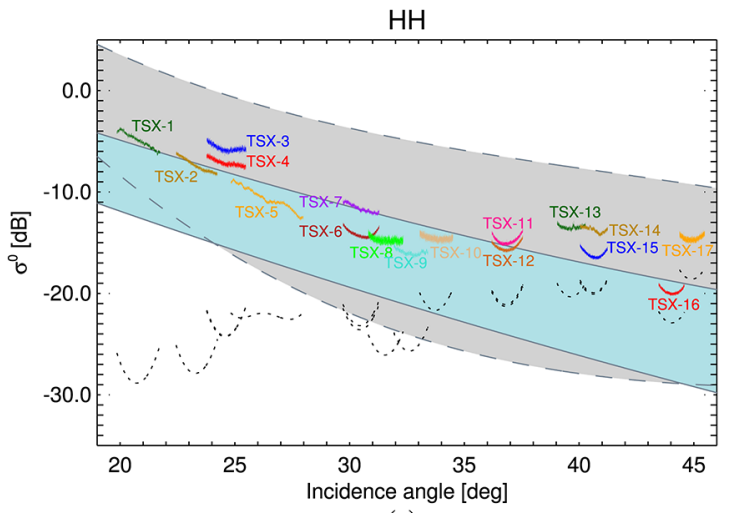

(a)

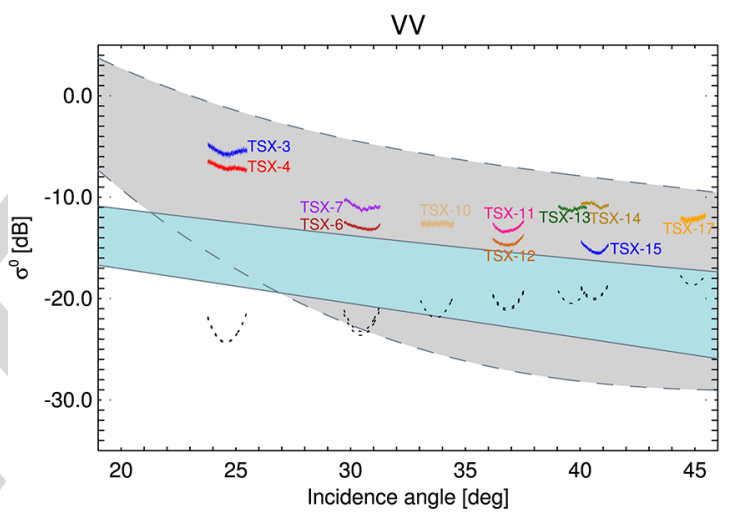

(b)

Fig. 2. Estimated $\sigma^{0}$ as a function of the incidence angle, using a sliding window in a range line basis with no overlap. (a) HH. (b) VV. The different data takes correspond to different colors; the (dotted black line) estimated NESZ is also plotted, and the shaded regions represent the predicted values from the NRL (delimited by dash-dot-dot lines) and XMOD2 (delimited by dashed lines) models, for a wind speed variation of $0.1-20.0 \mathrm{~m} / \mathrm{s}$ (average value between upwind, crosswind, and downwind is performed for XMOD2 and an average over the different PR models for $\mathrm{HH}$ extraction).

viations are 1.12, 1.23, and $2.63 \mathrm{~dB}$ for T-PR, E-PR, and X-PR, 270 respectively. This divergence between the VV and $\mathrm{HH}$ returns 271 (also among the different PR models) has been observed when 272 retrieving wind speeds from TSX/TDX VV-only data and HH- 273 only data as noted in [6], exploiting XMOD2 and PR models. 274

Since the available information of the sea conditions does not 275 correspond precisely to the time of acquisition, some error could 276 be introduced in the inversion of $\sigma^{0}$ for the theoretical models. 277 The NRL parametrization uses only two Nathanson tables [21], 278 at $30^{\circ}$ and $60^{\circ}$ of incidence angle, whereas five tables are used 279 to model reflectivity at incidence angles greater than $80^{\circ}$ [20]. 280 Therefore, a degradation in properly modeling $\sigma^{0}$ is expected 281 when considering typical spaceborne geometries with $20^{\circ}-45^{\circ} .282$

In case of XMOD2, which agrees much better with the 283 estimated values, the source of discrepancy should be also 284 related to the fact that this model has been optimized for a 285 given set of TSX/TDX copolarized acquisitions, not for quad- 286 pol data. Moreover, the quality of such experimental data (DRA 287 operation) should be carefully reviewed, particularly taking 288 into account that some calibration issues have been highlighted 289 when retrieving the fore and aft signals from the sum and 290 difference ones [4]. The calibration procedure proposed in [4] 291 has been applied for the along-track interferometric mode, 292 where the receive polarizations of the two halves of the antenna 293 
TABLE III

Statistical Distributions Used to Fit The SEA Clutter AMPLITUDE $Y$ (REFERENCES TO THE ESTIMATION METHODS ARE INDICATED)

\begin{tabular}{|c|c|c|c|}
\hline Distribution & Probability density function (PDF) & Parameters & Ref. \\
\hline \hline Log-normal & $\left.p_{Y}(Y)=\frac{1}{Y \sqrt{2 \pi \sigma^{2}} \exp \left(-\frac{(\ln Y-\mu)^{2}}{2 \sigma^{2}}\right)}\right)$ & $\begin{array}{l}\mu \text { (location) } \\
\sigma \text { (scale) }\end{array}$ & {$[12]$} \\
\hline Weibull & $p_{Y}(Y)=\beta \frac{Y^{\beta-1}}{a^{\beta}} \exp \left(-\left(\frac{Y}{a}\right)^{\beta}\right)$ & $\begin{array}{l}\beta \text { (shape) } \\
a \text { (scale) }\end{array}$ & {$[22]$} \\
\hline K-distribution & $p_{Y}(Y)=\frac{4 b \frac{(v+1)}{2} Y^{v}}{\Gamma(v)} K_{v-1}(2 Y \sqrt{b})$ & $\begin{array}{l}v \text { (shape) } \\
b \text { (scale) }\end{array}$ & {$\left[\begin{array}{l}{[23]} \\
{[24]}\end{array}\right.$} \\
\hline
\end{tabular}

${ }^{1} \Gamma(\cdot)$ is the gamma function and $K_{n}(\cdot)$ refers to the modified Bessel function of second kind and order $n$.

294 are the same, but no investigations have been made for the 295 quad-pol case (different polarizations in the fore and aft halves). 296 Hence, and as stated in the TSX product specification [7], 297 the DRA specific instrument characteristics have to be further 298 investigated for full calibration. From these results, it is clear 299 that an optimization of the XMOD2 and different PR models 300 is needed for quad-polarized data, using a wide data set with 301 in situ buoy measurements and considering further efforts in 302 instrument calibration for the fully polarimetric operation.

\section{3}

\section{Statistical Analysis}

304 For low-resolution radars and grazing angles typically above $30510^{\circ}$ (incidence angles less than 80 ), the amplitude (or magni306 tude) statistics of the sea returns have a "speckle"-like pattern 307 [8], [11], i.e., Rayleigh distributed in amplitude. For higher 308 resolution systems, the statistical description of the echo returns 309 deviates from the Gaussian hypothesis, having a target-like 310 response with more heavy-tailed distributions [8], [11]. This 311 non-Gaussian clutter has been commonly described, among 312 others, by log-normal and Weibull distributions [12], [13]. $313 \mathrm{~K}$-distribution, which provides also a good description of the sea 314 clutter magnitude for high-resolution radars [8], [11], has been 315 theoretically justified on the basis of the compound model [8].

316 Table III lists the probability density functions (PDFs) and 317 the related parameters for the log-normal, Weibull, and K distri318 butions. $Y$ refers to the amplitude (absolute value) of the single319 look $i$ th polarimetric channel $S_{i}$ (complex data), either $S_{\mathrm{HH}}$, $320 S_{\mathrm{VV}}$, or $S_{\mathrm{HV}}$ (polarization states in reception and transmission 321 are indicated by the first and second subindexes, respectively). 322 Fig. 3(a) and (b) show the fitting of the considered distribu323 tions (K, Rayleigh, log-normal, and Weibull) to the $S_{\mathrm{HH}}$ chan324 nel's magnitude for data takes TSX-4 and TSX-17, respectively, 325 which correspond accordingly to high-SNR (16.3 dB) and low$326 \mathrm{SNR}(3.7 \mathrm{~dB})$ scenarios. Only the results for a specific single 327 sub-AOI are shown, i.e., in this case, the data distributions are 328 computed using all the azimuth samples for a specific single 329 range bin. The distributions are plotted on a semilogarithm 330 scale, i.e., the clutter is expressed in decibels, which, for 331 calibrated data, refers to $\sigma^{0}$ (theoretical distribution fitting and 332 parametric estimation done in the linear domain).

333 For TSX-4, in the small incidence angle region, K-distribution 334 (dotted blue line with circle markers) fits best the data dis335 tribution (solid red line), particularly in the distribution's tail. 336 This is crucial, for instance, in GMTI operation, since the tail's 337 shape determines the detection threshold for a given false alarm

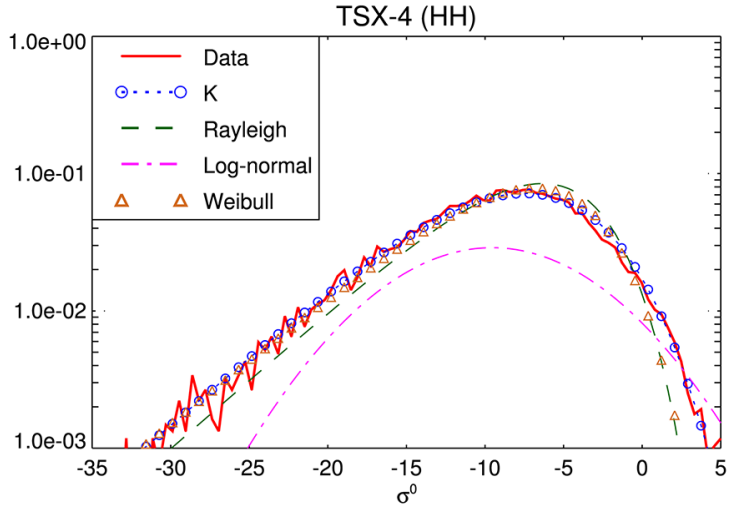

(a)

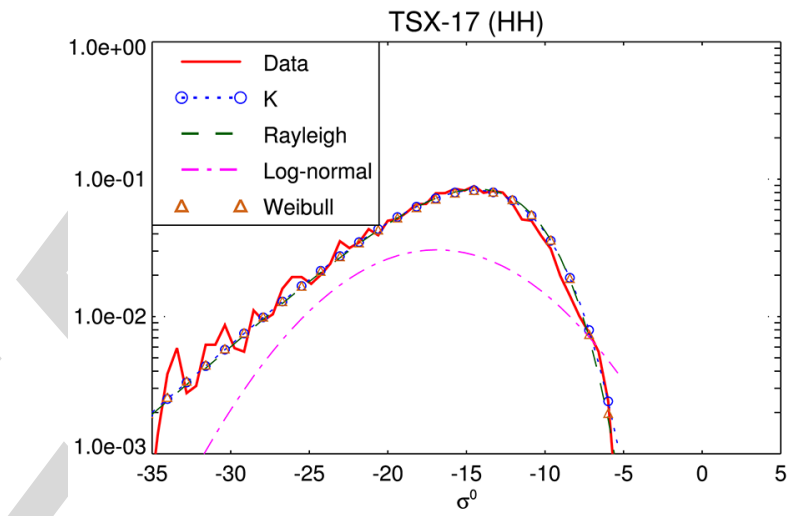

(b)

Fig. 3. Fitting of theoretical distributions (K, Rayleigh, log-normal, and Weibull) to the $S_{\mathrm{HH}}$ channel's magnitude (within a specific sub-AOI) for data takes (a) TSX-4 [SNR of $16.3 \mathrm{~dB}$ ] and (b) TSX-17 [SNR of $3.7 \mathrm{~dB}$ ].

rate. Weibull distribution shows good agreement with the data 338 only for smaller $\sigma^{0}$ values, whereas the log-normal does not fit 339 the data distribution. Similar results are obtained for the $S_{\mathrm{VV}} 340$ channel. In the cross-polar channel $S_{\mathrm{HV}}$, the three distributions 341 (K, Rayleigh, and Weibull) collapse to a complex Gaussian 342 distribution mainly due to the noise impact. For TSX-17, 343 the reduction in SNR, in comparison with TSX-4, leads to a 344 major contribution of the thermal noise, which is translated 345 into a more Gaussian-like data distribution. This behavior can 346 be recognized from the statistical fitting in Fig. 3(b), where 347 the $\mathrm{K}$ - and Weibull distributions tend to a Rayleigh one, i.e., 348 shape parameters $v>30$ and $\beta \approx 2$, accordingly. For the $S_{\mathrm{VV}} 349$ channel, there is an increase around $3 \mathrm{~dB}$ in SNR compared 350 with $\mathrm{HH}$ polarization, and K-distribution slightly departs from 351 Gaussianity, fitting best the data.

352

A good metric that allows deciding whether the data under 353 analysis are spikier or tend to a Gaussian distribution is the 354 shape parameter of the K-distribution, i.e., $v$. In Fig. 4, this 355 parameter is shown as a function of incidence angle for the 356 different data takes and copolar channels. ${ }^{5}$ Blacknell's method 357 has been used to estimate the shape and scale parameters of 358 the K-distribution. Similar results have been obtained using the 359 fractional moments method [23].

360

In the $S_{\mathrm{HH}}$ channel [see Fig. 4(a)], and for incidence angles 361 between $20^{\circ}$ and $33^{\circ}$, the shape parameter $v$ has a range of 362

${ }^{5}$ For those data takes at incidence angle above $35^{\circ}$ and with reduced SNR only TSX-13 and TSX-14 are shown in Fig. 4 for clarity in the representation. 


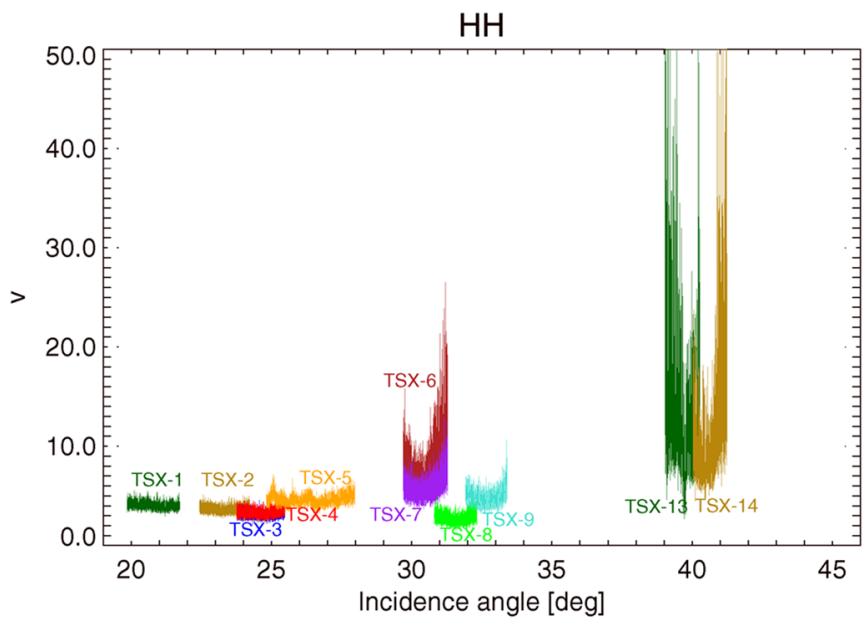

(a)

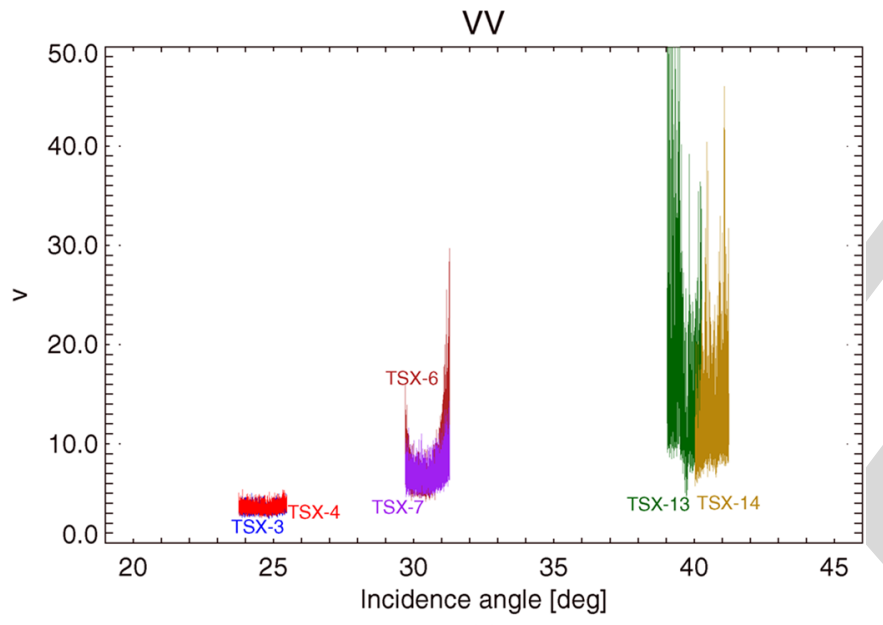

(b)

Fig. 4. Estimated shape parameter for a K-distributed amplitude fitting of the data as a function of the incidence angle, using a sliding window in a range line basis with no overlap. (a) HH. (b) VV. The different data takes are color-coded as in Fig. 2 (shape parameter estimated using Blacknell's approach).

363 3-10, with contained variation for each data take. For incidence 364 angles greater than $36^{\circ}$, the values of the shape parameter are 365 above 10, with much higher dispersion, related to the reduction 366 in SNR as incidence angle increases [see Fig. 2(a) and Table II]. 367 This is quite evident for TSX-16 and TSX-17, where noise 368 impact produces a variation on the shape parameter from 10 to 369 1000. For VV polarization [see Fig. 4(b)], similar trends are ob370 served, but in this case, the higher sea clutter returns in the high 371 incidence region reduce the shape parameter dispersion on data 372 take TSX-17, with values between 5 and 20. Comparing the 373 shape parameters of the polarimetric channels, different values 374 are obtained showing that the scalar product model (on which $375 \mathrm{~K}$-distribution is based) can be extended to a multivariative 376 product model, associating an individual texture variable per 377 polarimetric channel. For the cross-polar channels, high values 378 of the shape parameter are obtained with a high dispersion due 379 to the low sea backscattering contribution (close to NESZ).

380 One way to quantitatively evaluate the non-Gaussian behav381 ior of the data is by computing the NIM of the $n$th order [8], i.e.,

$$
\mathrm{NIM}_{n}=\frac{E\left\{Z^{n}\right\}}{E^{n}\{Z\}}
$$

where $Z$ refers to data intensity (or power), i.e., $Z=Y^{2}$, being 382 $Y=\left|S_{i}\right|$ the magnitude, and $E\{\cdot\}$ is the expectation operator. 383 Values of the $n$ th-order NIM above $n$ ! (Gaussian limit) are 384 indicators of spiky data.

In general, and for the copolar channels, the estimated 386 NIM (as a function of the estimated K-distribution shape pa- 387 rameter $v$ ) follows quite good the theoretical K-distribution 388 NIM trend, for the third and fourth orders, when considering 389 small to medium values of $v(<10)$, i.e., spikier clutter re- 390 turns. Therefore, the compound model theory that supports the 391 $\mathrm{K}$-distribution is able to describe the mechanism, which results 392 from a combination of Bragg scattering (from resonant capil- 393 lary waves) and whitecap scattering originated from breaking 394 waves [8]. The presence of the latter phenomena is known to 395 increase the spikiness of the clutter returns [8], associated to 396 smaller values of $v$ as observed for data takes with incidence an- 397 gle below $30^{\circ}$, with medium to high SNR conditions ( $\left.>10 \mathrm{~dB}\right), 398$ as indicated in Table II.

Analogously, the estimated third- and fourth-order NIM 400 have been compared with the theoretical ones for Weibull and 401 log-normal distributions. Weibull presents, generally, a worse 402 matching with the theoretical trends for the copolar channels 403 compared with the K-distribution. When considering a log- 404 normal distribution fitting, a very poor matching between the 405 estimated NIM and the theoretical ones is obtained. In sum- 406 mary, K-distribution provides good fitting for specific TSX 407 acquisitions over the sea, particularly for low incidence angles, 408 where SNR conditions are favorable $(>10 \mathrm{~dB})$. In this region, 409 the lowest values of the K-distribution shape parameter are 410 obtained, indicating a much spikier behavior of the data, which 411 can be also related to the higher contribution of breaking wave 412 events, as analyzed in Section VI.

$\mathrm{K}$-distribution is among one of the statistical distributions 414 used to model and describe non-Gaussian statistics, based on 415 the well-known compound model. Under this hypothesis, the 416 complex signal can be characterized as a product between 417 the square root of a random variable $T$ (representing the tex- 418 ture) and an independent zero-mean complex Gaussian random 419 process ("speckle"). In this sense, the computation of the so- 420 called log-cumulants is very useful to evaluate the presence of 421 texture, i.e., non-Gaussian statistics [9]. Such analysis is typ- 422 ically performed through the log-cumulant diagram [9], [10], 423 where the second $\kappa_{2}$ (variance) and third $\kappa_{3}$ (skewness) log- 424 cumulants are plotted against each other. These log-moments 425 are computed as [10]

$$
\begin{aligned}
& \kappa_{2}=m_{2}-m_{1}^{2} \\
& \kappa_{3}=m_{3}-3 m_{1} m_{2}+2 m_{1}^{3}
\end{aligned}
$$

where $m_{n}$ is the log-moment of order $n$, i.e., $m_{n}=427$ $1 / L \sum_{l=1}^{L}\left(\log Z_{l}\right)^{n}$, where $L$ intensity samples have been used. 428

Fig. 5 shows the log-cumulant diagram for the three rep- 429 resentatives acquisitions considered in Fig. 1 (from near to 430 far incidence angle regions). These plans are covered by five 431 (texture) distribution families, namely, Gamma (dash-dotted 432 line modeling a K-distribution in amplitude), inverse Gamma 433 (dashed black line), Beta (light gray shaded region), inverse 434 


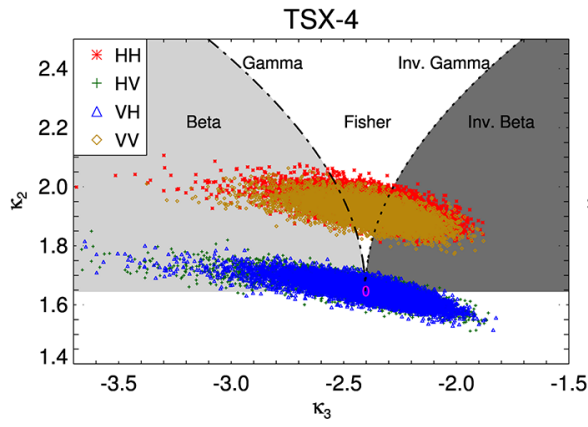

(a)

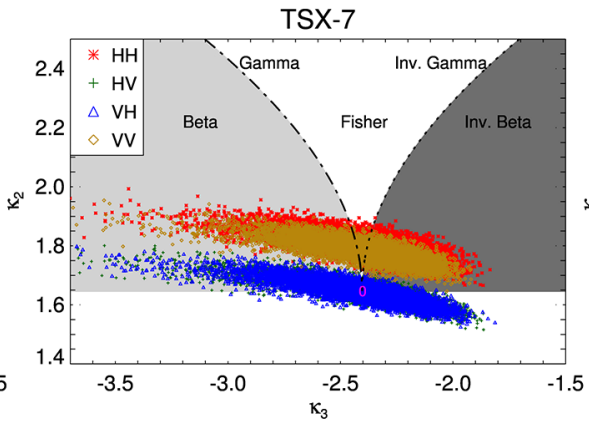

(b)

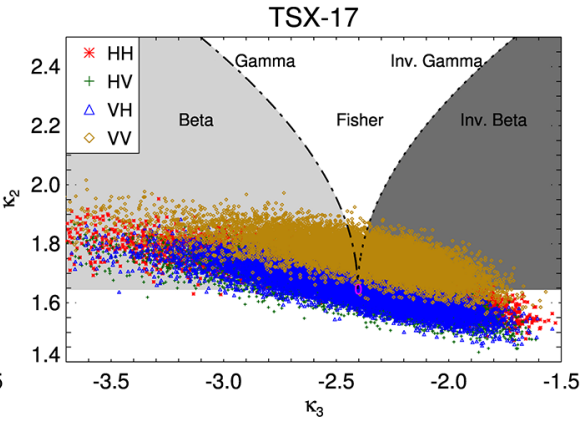

(c)

Fig. 5. Log-cumulant diagrams for different data takes: (a) TSX-4, (b) TSX-7, and (c) TSX-17. A sliding window in a range line basis with no overlap has been used (HH as red "x," HV as green "+," VH as " $\triangle$," and VV as gold " $\diamond ")$.

435 Beta (gray shaded region on the right), and Fisher (region in 436 between). The intersection point between these distributions 437 corresponds to no texture at all, i.e., Gaussian statistics.

438 The results in Fig. 5 are in line with the previous statistical 439 analysis: for TSX-4, in the near incidence angle region, the 440 copolar channels lie higher up in the diagram compared with 441 the cross-polar channels, indicating a deviation from Gaussian 442 statistics (spikier response). As the incidence angle increases, 443 the copolar channels move to lower regions in the diagram 444 (less texturized). For TSX-17, with the highest incidence angle, $445 \mathrm{VV}$ has more texture compared with $\mathrm{HH}$, as expected from the 446 K-distribution shape parameter study.

\section{7 \\ 448 \\ VI. Analysis of the Polarimetric AND SCATTERING FEATURES}

\section{A. SAR Polarimetry}

450 A polarimetric SAR (PolSAR) system measures the scatter451 ing matrix, which, for the monostatic case and on the Pauli 452 basis, can be expressed in vectorial notation as

$$
\mathbf{k}=\frac{1}{\sqrt{2}}\left[\begin{array}{lll}
S_{\mathrm{HH}}+S_{\mathrm{VV}} & S_{\mathrm{HH}}-S_{\mathrm{VV}} & 2 S_{\mathrm{HV}}
\end{array}\right]^{T}
$$

453 where $(\cdot)^{T}$ refers to the transpose operator, and $S_{i}$ are the com454 plex data of the $i$ th polarimetric channel. The distribution of $\mathbf{k}$ is 455 completely described by the Hermitian positive definite coher456 ency matrix $\mathbf{T}=E\left\{\mathbf{k k}^{H}\right\}$ under the Gaussian assumption [25], 457 where $(\cdot)^{H}$ is the transpose and complex conjugate operator.

458 The coherency matrix $\mathbf{T}$ is estimated from the data, such that 459 the speckle noise is filtered. The maximum-likelihood estimator 460 can be obtained as a spatial averaging (boxcar or multilook filter)

$$
\widehat{\mathbf{T}}=\frac{1}{L} \sum_{i=1}^{L} \mathbf{k}_{i} \mathbf{k}_{i}^{H}
$$

461 where $L$ refers to the number of looks or samples employed in 462 the estimation of $\mathbf{T}$, and $\mathbf{k}_{i}$ is the vector in the Pauli basis for 463 the $i$ th sample.

\section{B. Features Definition}

465 The main objective of the polarimetric study is to analyze the 466 scattering properties of the sea surface observed by the high467 resolution X-band TSX sensor. This way, two different sets of polarimetric features are considered. First, the eigendecompo- 468 sition of the coherency matrix allows extracting roll-invariant 469 parameters, such as the entropy $H$, anisotropy $A$, and mean al- 470 pha angle $\bar{\alpha}$, as originally proposed by Cloude and Pottier [14]. 471 $H$ measures the randomness of the scattering mechanism, $\bar{\alpha} 472$ provides physical information about the type of average scatter, 473 and $A$ (complementary to $H$ ) measures the relative significance 474 of the second and third eigenvalues. The $H / A / \bar{\alpha}$ features allow 475 a physical interpretation of the PolSAR data, and thus, their 476 correct estimation provides a reliable prediction of physical 477 parameters [5], [14]. This eigendecomposition-based analysis 478 has been successfully applied over the oceans to determine 479 polarimetric scattering mechanisms and for discrimination/ 480 classification of different types of slicks [16], [26], [27]. 481

To complement the eigendecomposition-based analysis, ad- 482 ditional polarimetric features exploiting the intensity of the 483 copolarized channels have been used, based on the $\sigma^{0}$ model 484 proposed by Kudryavtsev et al. [15]. As analyzed in [15], 485 [28], and [29], the Bragg theory is not fully able to explain 486 and represent the backscattering from the ocean, due to the 487 existence of sea spikes (in high-resolution radar data), which 488 induce large deviations of observed PR compared with the 489 theoretical Bragg one. This suggested that some mechanism 490 supporting non-Bragg scattering and connected with breaking 491 waves also contributes to the NRCS from the sea. The proposed 492 NRCS model in [15] is a superposition (in linear units) of a 493 regular sea surface, polarized component related to the Bragg 494 scattering $\left(\sigma_{p p}^{0 B}\right)$, and surface areas with enhanced roughness 495 produced by breaking waves, nonpolarized component linked 496 to non-Bragg scattering $\left(\sigma_{p p}^{0 n B}\right)$, i.e., $\sigma_{p p}^{0}=\sigma_{p p}^{0 B}+\sigma^{0 n B}$. It is 497 assumed that the nonpolarized component is the same for both 498 copolar channels.

As anticipated by several studies [15], [28], [29], the contri- 500 bution of the nonpolarized radar returns to the total NRCS can 501 be considerable, mainly due to the presence of breaking waves. 502 As suggested in [15] and [29], the computation of the PR 503

$$
\mathrm{PR}=\frac{\sigma_{\mathrm{HH}}^{0}}{\sigma_{\mathrm{VV}}^{0}}=\frac{\sigma_{\mathrm{HH}}^{0 B}+\sigma^{0 n B}}{\sigma_{\mathrm{VV}}^{0 B}+\sigma^{0 n B}}
$$

is an indicator of the presence of significant wave breaking 504 $(\mathrm{PR} \approx 1)$, deviating from the $\mathrm{PR}$ for the Bragg component 505 $p_{B}=\sigma_{\mathrm{HH}}^{0 B} / \sigma_{\mathrm{VV}}^{0 B}$. In this paper, $p_{B}$ is computed as the ratio 506 
507 between the perpendicular $\left(B_{\perp}\right)$ and parallel $\left(B_{\|}\right)^{6}$ Bragg 508 scattering coefficients according to [5]. It must be noted that 509 the effect of slope of the long tilting waves on the NRCS is 510 higher at the $\mathrm{HH}$ polarization than in $\mathrm{VV}$, particularly at large 511 incidence angles [15], and hence, this could lead to an increase 512 in PR not directly related to the breaking waves. Nevertheless, 513 it is complicated to separate the effect of breaking waves from 514 tilting waves, since, in the formation process of the former 515 ones, there is a progressive increase of the slope (generation 516 of a sloping wave front) correlated with a temporal increase 517 of the surface roughness [16]. In this sense, further studies 518 are required to carefully analyze the impact of both tilting and 519 breaking waves, trying to separate both processes.

\section{C. X-Bragg and Extended X-Bragg (X2-Bragg) Models}

521 It has been generally assumed that the radar sea echo returns 522 are mostly dominated by Bragg surface scattering; however, 523 some depolarization has been observed, as noted in [16], [26], 524 and [30]. Such nonpolarized returns have been found to sig525 nificantly contribute to the sea backscattering due to breaking 526 waves (increasing surface roughness), as analyzed in [15], 527 [28], and [29], particularly when increasing the frequency of 528 operation (and thus the resolution) [15].

529 Under these considerations, the application of the small per530 turbation model (SPM) for the polarimetric scattering analysis 531 would be too simplistic, as its validity range is limited to small 532 surface roughness conditions [5]. Therefore, the evaluation of 533 the X-Bragg model, originally proposed by Hajnsek et al. [5], 534 seems to be more reasonable, since it is a two-component 535 model, including a Bragg scattering term and a roughness in536 duced rotation symmetric disturbance. This way, depolarization 537 (nonpolarized components) and cross-polarized backscattering 538 effects are included. As proposed in [5] and [16], this can 539 be obtained modeling the surface as a reflection symmetric 540 depolarizer, randomly rotating the Bragg coherency matrix $\mathbf{T}$ 541 about an angle $\beta$ w.r.t. the local surface normal and averaging it 542 over a statistical distribution $p_{\beta}(\beta)$. For a uniform distribution $543 p_{\beta}(\beta)$ with zero mean and width $\beta_{1}$, the coherency matrix can 544 be expressed by [5]

$$
\mathbf{T}=m_{s}^{2}\left[\begin{array}{ccc}
C_{1} & C_{2} \operatorname{sinc}\left(2 \beta_{1}\right) & 0 \\
C_{2}^{*} \operatorname{sinc}\left(2 \beta_{1}\right) & C_{3}\left(1+\operatorname{sinc}\left(4 \beta_{1}\right)\right) & 0 \\
0 & 0 & C_{3}\left(1-\operatorname{sinc}\left(4 \beta_{1}\right)\right)
\end{array}\right]
$$

545 where $m_{s}$ is the backscatter amplitude related to the surface 546 roughness; sinc function refers to $\operatorname{sinc}(x)=\sin (x) / x$; and the 547 coefficients $C_{1}, C_{2}$ and $C_{3}$ can be expressed in terms of $B_{\perp}$ and $548 B_{\|}[5]$. These coefficients depend on the incidence angle $\gamma$ and 549 the dielectric constant $\epsilon=\epsilon^{\prime}-\jmath \epsilon^{\prime \prime}$. The latter can be obtained 550 from the model developed by Klein and Swift [31].

551 The width of the distribution $\beta_{1}$ models the roughness dis552 turbance of the surface and controls the level of cross-polarized 553 power and the polarimetric $\left(S_{\mathrm{HH}}+S_{\mathrm{VV}}\right)\left(S_{\mathrm{HH}}-S_{\mathrm{VV}}\right)$ coher554 ence [5]: for $\beta_{1}=0^{\circ}$ (smooth surface), the cross-polarized

\footnotetext{
${ }^{6}$ Note that, here, perpendicular and parallel are referenced w.r.t. the incidence plane.
}

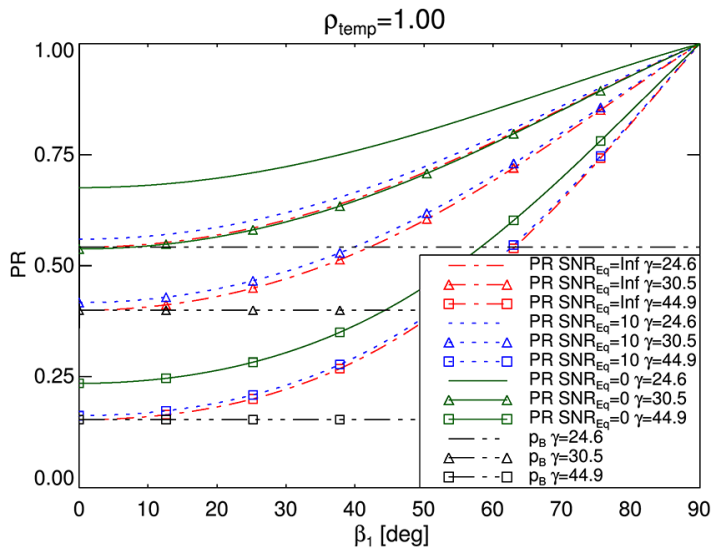

Fig. 6. PR of the X-Bragg $\left(\mathrm{SNR}_{\mathrm{Eq}}=\infty\right)$ and X2-Bragg models is represented as a function of the roughness $\beta_{1}$ parameter for different incidence angles $\gamma$ and different noise conditions $\mathrm{SNR}_{\mathrm{Eq}}$ (an X-band system at $9.65 \mathrm{GHz}$, a sea temperature of $10^{\circ} \mathrm{C}$, and salinity of $35 \%$ are considered).

power is zero, and there is no depolarization effect (corre- 555 sponding to the "pure" Bragg or SPM model); with increasing 556 $\beta_{1}$ (increasing roughness), the $\mathrm{HV}$ term increases, and the 557 $\left(S_{\mathrm{HH}}+S_{\mathrm{VV}}\right)\left(S_{\mathrm{HH}}-S_{\mathrm{VV}}\right)$ coherence decreases until 0 in the 558 high roughness limit case of $\beta_{1}=90^{\circ}$ (azimuthally symmetric 559 surface). The breaking waves produce a temporal increase in 560 the surface roughness [8], [16], and this could be related to an 561 increase also in the $\beta_{1}$ term of the X-Bragg model. In fact, this 562 model has been used to derive a description of the polarimetric 563 behavior of a breaking wave, as presented in [16]. Therefore, 564 it is interesting to evaluate the applicability of the X-Bragg 565 model to SAR polarimetric sea data, which can be useful for 566 future studies trying to invert some geophysical parameters of 567 the ocean using polarimetric data.

The DRA acquisition mode [4], employed to obtain fully po- 569 larimetric images with an along-track interferometric configu- 570 ration, has a two-folded impact on measured data. On one hand, 571 as the receive antenna is halved, a direct consequence is a reduc- 572 tion on the received gain, which translates into higher values of 573 NESZ. On the other hand, the alternate transmission of horizon- 574 tal and vertical polarizations in a pulse-by-pulse basis jointly 575 with the along-track configuration (to receive simultaneously 576 $\mathrm{H}$ and $\mathrm{V}$ ) produces a temporal lag between different pairs of 577 channels. The different polarimetric channels should be spatial 578 aligned (coregistered), such that they observe the scene from 579 the same positions but at different times. During this time lag, 580 around $\tau_{\text {coreg. }}=1 \mathrm{~ms}$ for TSX $S_{\mathrm{HH}}$ and $S_{\mathrm{VV}}$ channels (or $S_{\mathrm{HV}} 581$ and $S_{\mathrm{VH}}$ ), the sea backscattered field decorrelates due to inter- 582 nal clutter motion. Typical decorrelation times of the sea are 583 between 8 and $10 \mathrm{~ms}$ for wind speeds of $15 \mathrm{~m} / \mathrm{s}$ down to $5 \mathrm{~m} / \mathrm{s}, 584$ [32]. For an X-band system at VV, the temporal coherence 585 $\rho_{\text {temp }}$ could be between 0.9 and 1.0 for a time lag around $1 \mathrm{~ms}, 586$ [32]. From the results presented in Section VI-D for TSX quad- 587 pol, it has been observed that both the noise level and the sea 588 clutter temporal decorrelation should be accounted for in the 589 $\mathrm{X}$-Bragg model to properly characterize the sea clutter in po- 590 larimetric terms. Taking into account these issues, the X-Bragg 591 model has been extended to the X2-Bragg model (extended- 592 extended Bragg). The mathematical derivation of the X2-Bragg 593 


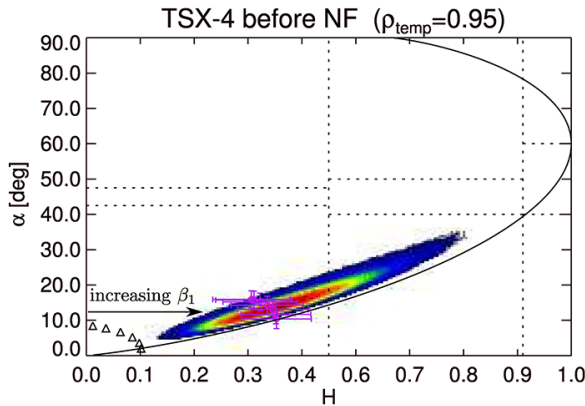

(a)

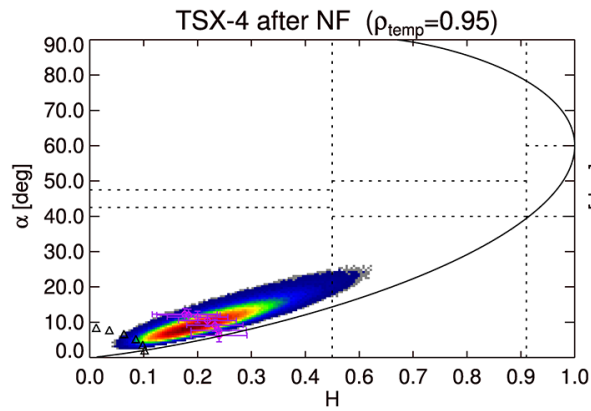

(d)

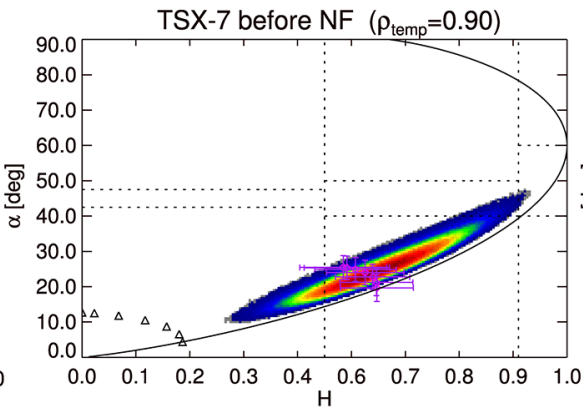

(b)

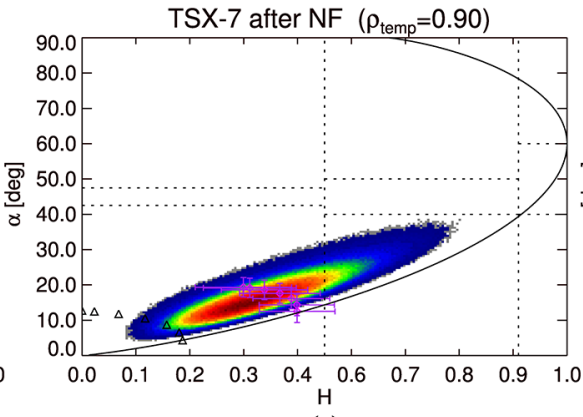

(e)

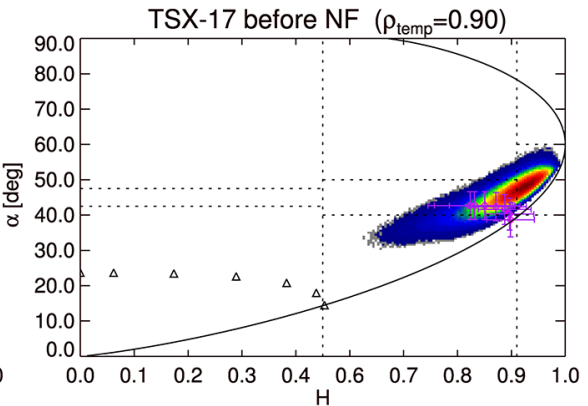

(c)

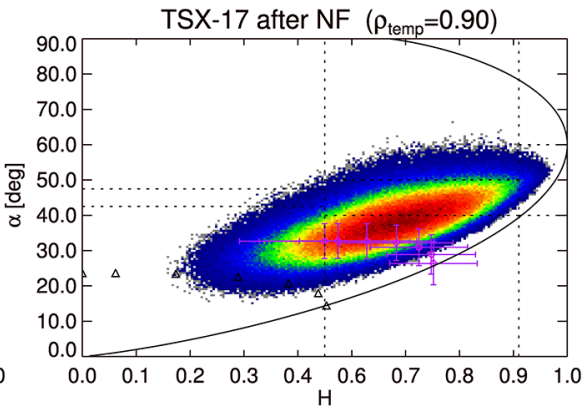

(f)

Fig. 7. Two-dimensional distributions on $H / \bar{\alpha}$ plane for different data takes (TSX-4, TSX-7, and TSX-17, from left to right), including the theoretical $\mathrm{X} / \mathrm{X} 2$-Bragg models $\left(\beta_{1}\right.$ variation from $0^{\circ}$ to $90^{\circ}$ and single dielectric constant for standard sea conditions of $10{ }^{\circ} \mathrm{C}$ and salinity of $35 \%$ ); black triangles

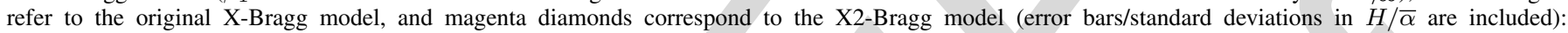
(a)-(c) before and (d)-(f) after NF. (a) and (b) TSX-4 with $\rho_{\text {temp }}=0.95$. (b) and (e) TSX-7 with $\rho_{\text {temp }}=0.90$. (c) and (f) TSX-17 with $\rho_{\text {temp }}=0.90$.

594 and a theoretical evaluation for different system/scenario con595 ditions are presented in Appendixes A and B, respectively.

596 In Fig. 6, the PR of the X-Bragg $\left(\mathrm{SNR}_{\mathrm{Eq}}=\infty\right)$ and the $597 \mathrm{X} 2$-Bragg is plotted as a function of $\beta_{1}$ for different incidence 598 angles $\gamma$ and effective ${ }^{7}$ signal-to-noise ratio $\mathrm{SNR}_{\mathrm{Eq}}$ conditions 599 (no temporal decorrelation has been considered). The horizon600 tal lines correspond to the PR for Bragg components $p_{B}$. It can 601 be seen how the PR approaches $p_{B}$ for $\beta_{1}$ close to zero (pure 602 Bragg) and increases to higher values as roughness condition 603 increases, with a value of 1 for complete depolarized scattering. 604 From [15] and [29], this trend can be related to higher contri605 bution of breaking wave events, which induce higher roughness 606 conditions. It must be also noted that, as the noise contribution 607 increases, there is also an increase in the PR, since the noise is 608 a common nonpolarized term affecting both copolar channels.

\section{D. Experimental Results}

610 An analysis of the scattering plots in the $H / \bar{\alpha}$ plane has been 611 carried out for the different data takes, without and with additive $612 \mathrm{NF}$ as proposed in [5]. Fig. 7 shows these 2-D distributions 613 for the three representative data takes (near to far incidence, 614 from left to right). Before NF, the dominant mechanism moves 615 from low entropy surface scattering 8 at near incidence [see 616 Fig. 7(a)], passing by medium entropy surface scattering [see 617 Fig. 7(b)], ending up to high entropy region characterized by 618 random anisotropic scatters [see Fig. 7(c)], caused by the higher 619 noise contribution. Comparatively, NF produces a reduction

\footnotetext{
${ }^{7}$ Please refer to Appendix A for further details on the definition of this equivalent $\mathrm{SNR}_{\mathrm{Eq}}$.

${ }^{8}$ This region corresponds to zone 9 as designated by Cloude and Pottier [14], including surface scattering and specular scattering phenomena.
}

both in $H$ and $\bar{\alpha}$, leading to a dominant scattering in the low 620 entropy surface zone $\left(H<0.5\right.$ and $\left.\bar{\alpha}<40^{\circ}\right)$ for data takes 621 with incidence angle below $36^{\circ}$ (e.g., TSX-4 and TSX-7). 622

The distributions of the eigenvalues in Fig. 8 for the three 623 reference data takes are a good visual indicator of the polarimet- 624 ric mechanisms' evolution as a function of incidence angle. In 625 Fig. 8, the lowest eigenvalue $\left(\lambda_{4}\right)$ has been also included since 626 it provides information on the expected noise floor, such that the 627 dominant mechanism's relative contribution with respect to the 628 noise can be easily tracked. For TSX-4 [see Fig. 8(a)], there is a 629 clear dominant mechanism $\left(\lambda_{1}\right)$, surface scattering, as observed 630 from the $\alpha_{1}$ distribution in Fig. 9(a), centered around $5^{\circ}$. As 631 incidence angle increases, the first eigenvalue decreases. At 632 the farthest available incidence angle (TSX-17) [see Fig. 8(c)], 633 no clear dominant mechanism is present, being the distributions 634 of the eigenvalues closer to each other, such that a noise-like 635 mechanism is expected in accordance with the $\sigma^{0}$ and statistical 636 characterizations.

In general, the second mechanism almost keeps its relative 638 strength w.r.t. noise floor independently of incidence angle. In 639 Fig. 9, where the distributions of the individual $\alpha$ parameters 640 are shown, the related $\alpha_{2}$ with a similar trend as $\alpha_{3}$ lies between 641 $70^{\circ}$ and $90^{\circ}$. These values are representative of double-bounce 642 scattering, less probable in the sea backscattering. Considering 643 the X-Bragg model extension to account only for thermal 644 noise impact, parameters associated to the second mechanism 645 do not agree with the observations from experimental data: 646 on one hand, $\alpha_{2}$ corresponds to $90^{\circ}$ independently from $\beta_{1} 647$ and $\mathrm{SNR}_{\mathrm{Eq}}$; on the other hand, the expected level of $\lambda_{2}$ is 648 much closer to $\lambda_{3}$ than that observed from real data. These 649 considerations reinforce the need to include also the impact 650 of sea clutter temporal decorrelation between the polarimetric 651 
TSX-4

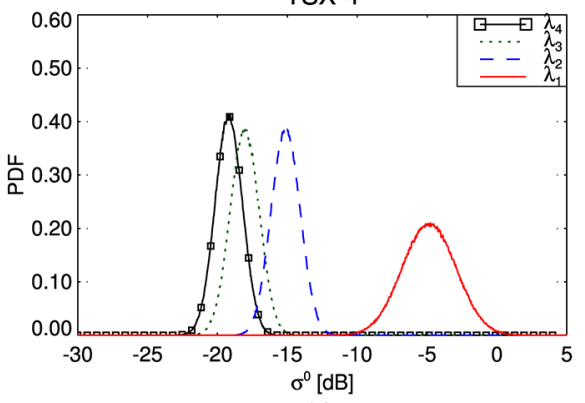

(a)
TSX-7

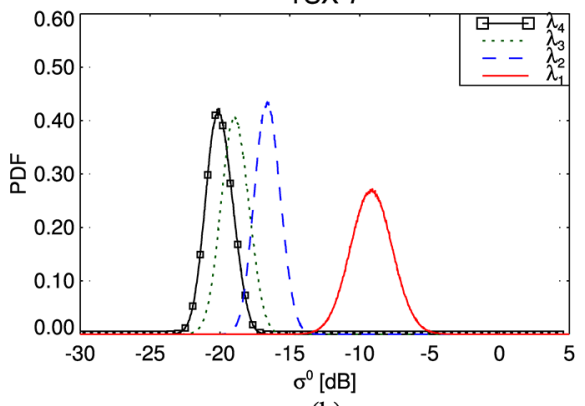

(b)

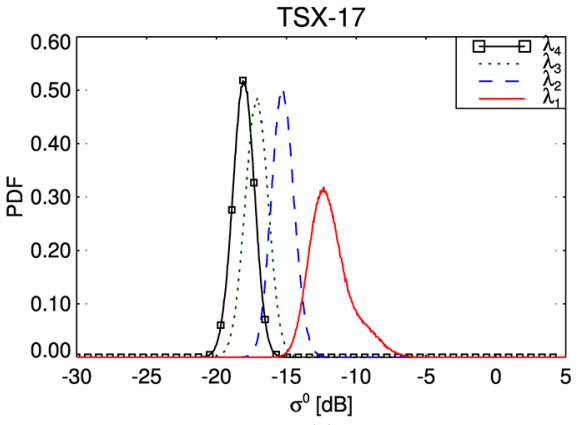

(c)

Fig. 8. Distribution of the eigenvalues, before NF, for different data takes. (a) TSX-4. (b) TSX-7. (c) TSX-17.

TSX-4

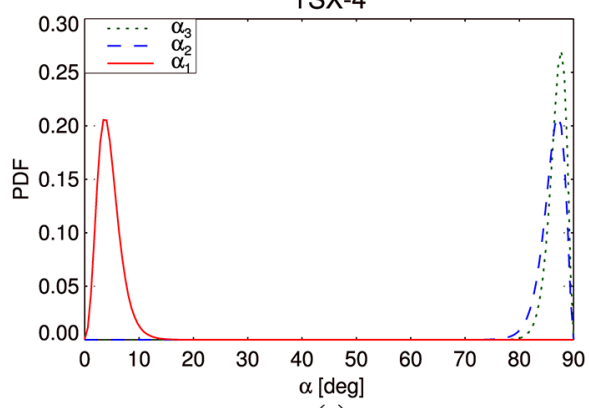

(a)

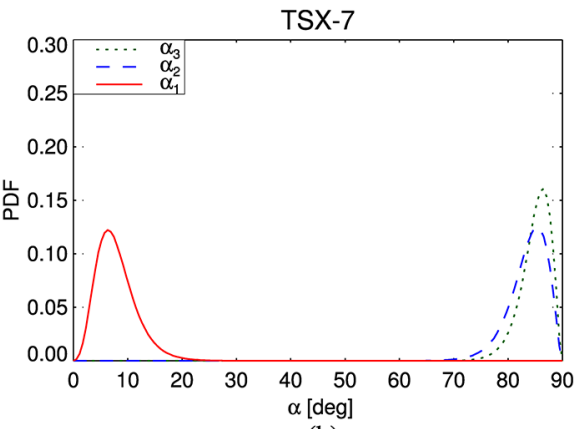

(b)

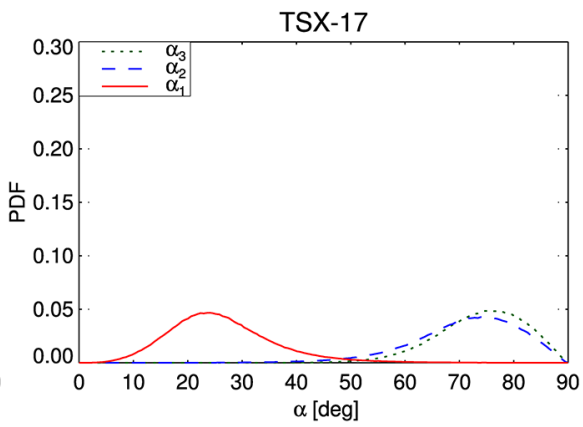

(c)

Fig. 9. Distribution of the alpha parameters, before NF, for different data takes. (a) TSX-4. (b) TSX-7. (c) TSX-17.

652 channels $\left(\rho_{\text {temp }}\right)$, intrinsically related to the DRA acquisition 653 mode itself, as proposed in the X2-Bragg model and described 654 in Appendixes A and B.

655 On top of the different $H / \bar{\alpha}$ distributions in Fig. 7, the 656 classical X-Bragg model, as black triangles, and the extended 657 model X2-Bragg, as magenta diamond symbols, are depicted 658 for different values of $\beta_{1}$ (from $0^{\circ}$ to $90^{\circ}$ in steps of $15^{\circ}$ ). 659 Standard deviations are also included for the X2-Bragg model 660 (related to $\mathrm{SNR}_{\mathrm{Eq}}$ estimation from the data). Standard sea 661 conditions have been assumed, i.e., $10^{\circ} \mathrm{C}$ and $35 \%$ salinity.

662 An important bias exists between the original X-Bragg model 663 and the data, after and, in particular, before NF. For X2-Bragg, 664 a much better matching between the theoretical model and the 665 experimental data is obtained. For TSX-4 [see Fig. 7(a)], a $666 \rho_{\text {temp }}$ of 0.95 provides a good matching between the theoretical 667 extended model and the data before NF, whereas for TSX-7 668 [see Fig. 7(b)], this value reduces to 0.90. In case of TSX-17 669 [see Fig. 7(c)], a higher bias is recognized, and reducing $670 \rho_{\text {temp }}$ below 0.9 does not provide any additional improvement: 671 from the theoretical analysis of the X2-Bragg model, higher 672 clutter decorrelation does not increase entropy for regions with $673 H>0.6$. Additive noise subtraction reduces entropy from 6740.92 to 0.7 , but the proposed extended model predicts an aver675 age alpha value below the one observed in the data. Therefore, 676 there might be an additional mechanism contributing to this 677 discrepancy, which is not considered in the X-Bragg model; 678 additional measurements with higher SNR conditions should 679 be analyzed to support this hypothesis.

680 The X2-Bragg model gets close to the centroid of the data 681 distributions in $H / \bar{\alpha}$ for values of $\beta_{1}$ different from zero, which means that no pure Bragg is being imaged, but there is a 682 depolarized term contributing also to the backscattering. An 683 increase in $\beta_{1}$ is related to higher surface roughness producing 684 depolarization and an increase in the entropy. To complement 685 the polarimetric study and gain additional insights into the 686 nonpolarized contribution of the sea backscattering, the PR for 687 the available quad-pol data takes is represented as a function of 688 the incidence angle in Fig. 10. The PR $p_{B}$ for the pure Bragg 689 model is also included as solid black line.

Before NF [see Fig. 10(a)], an important difference between 691 the estimated PR and $p_{B}$ exists, which means that an important 692 contribution on nonpolarized returns is presented, probably 693 related to breaking wave effects. Even for the high incidence 694 region (above $36^{\circ}$ ), where Bragg scattering is expected to be 695 dominant, as stated in [10], an appreciable divergence between 696 the PRs is observed (e.g., TSX-15 and TSX-17). Nevertheless, 697 as pointed out throughout this paper, the reduced SNR condi- 698 tions $(\approx 3-4 \mathrm{~dB})$ can explain this effect, since the thermal noise 699 is also a nonpolarized component common to both copolar 700 channels. This can be recognized from Fig. 10(b), where the 701 difference between PR and $p_{B}$ has been reduced for the high 702 incidence angle region after NF. ${ }^{9}$

703

Following the two-component NRCS model (Bragg/non- 704 Bragg) proposed by Kudryavtsev et al. [15], the relative 705

\footnotetext{
${ }^{9}$ In Fig. 10, the images have been radiometrically calibrated before the application of any further processing, i.e., the NESZ has been subtracted (in linear units) from the calibrated digital pixels, as proposed in [18]. From these results, it can be seen that there is still some residual noise that should be filtered out and that quad-pol data provide the means to do so.
} 


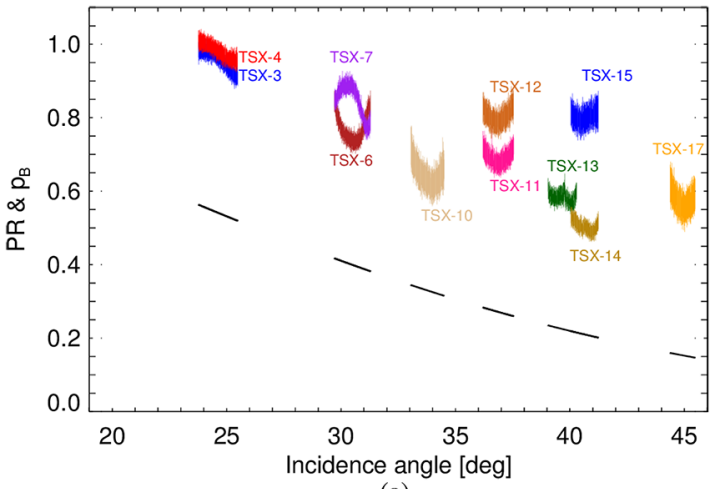

(a)

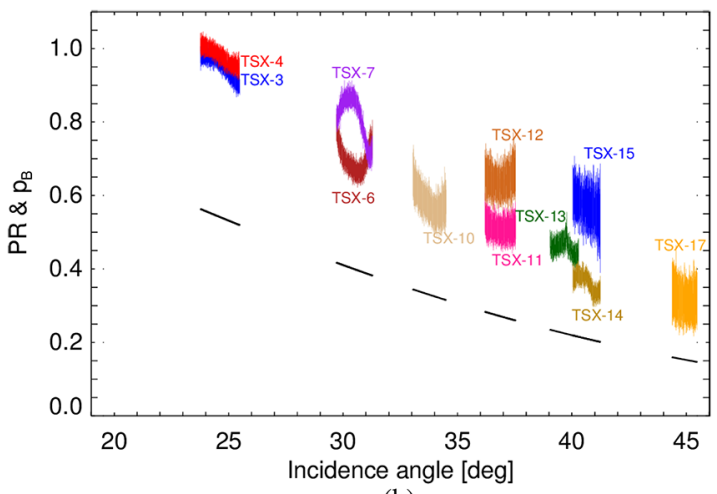

(b)

Fig. 10. PR as a function of the incidence angle for the different quad-pol data takes (color-coded as in Fig. 2): (a) before and (b) after NF; solid black lines refer to the PR for pure Bragg $p_{B}$.

706 contribution of the non-Bragg component with respect to the 707 total backscattered power for the $S_{\mathrm{HH}}$ channel $\left(\sigma^{0 n B} / \sigma_{\mathrm{HH}}^{0}\right)$ has 708 been computed, and it ranges from $50 \%$ to $90 \%$ (high to small 709 incidence angles), whereas for $\mathrm{VV}$, the figures $\left(\sigma^{0 n B} / \sigma_{\mathrm{VV}}^{0}\right)$ are 710 around $20 \%-70 \%$. These results are in line with the studies 711 carried out in [15] and [29]. For TSX-3 and TSX-4, $\sigma^{0 n B} / \sigma_{\mathrm{VV}}^{0}$ 712 and $\sigma^{0 n B} / \sigma_{\mathrm{HH}}^{0}$ have very similar trends close to the $90 \%$ of the 713 total backscattered power. In this very case, with the smallest 714 incidence angle $\left(25^{\circ}\right)$, specular returns may partially contribute 715 to the non-Bragg scattering [10], apart from possible breaking 716 wave effects.

717 A fitting of the X-Bragg and X2-Bragg models to the mea718 sured PR has been carried out. After NF, $\beta_{1}$ values providing 719 the minimum average error (between the models and the data) 720 within each quad-pol acquisition are summarized in Table IV. 721 As described in Section VI-B, an increase in $\beta_{1}$ is related 722 to a roughness enhancement and thus a higher depolarization 723 contribution. From Table IV, it can be generally stated that $\beta_{1}$ 724 reduces as a function of the incidence angle in accordance to 725 the decrease of the nonpolarized component. For the X2-Bragg 726 model, $\beta_{1}$ corresponds to the average value for three differ727 ent temporal decorrelation conditions assumed in the model $728\left(\rho_{\text {temp }}=1.0,0.95,0.90\right)$. Due to the presence of thermal noise 729 and possible temporal decorrelations, the X-Bragg model over730 estimates the surface roughness, when compared with the pro731 posed X2-Bragg model, particularly for the high incidence 732 region (above $36^{\circ}$ ).
TABLE IV

$\beta_{1}$ (Roughness) Providing the Minimum Average Error Between Estimated PR From X/X2-Bragg DATA FitTing AND MEAsured PR FOR QUAD-POL ACQUisitions AFTER NF

\begin{tabular}{|c|c|c|}
\hline ID & X-Bragg & X2-Bragg \\
\hline TSX-3 & 84 & 83.3 \\
\hline TSX-4 & 87 & 87 \\
\hline TSX-6 & 59 & 56 \\
\hline TSX-7 & 73 & 72 \\
\hline TSX-10 & 51 & 46.7 \\
\hline TSX-11 & 51 & 46.7 \\
\hline TSX-12 & 63 & 62 \\
\hline TSX-13 & 50 & 44.7 \\
\hline TSX-14 & 41 & 32.7 \\
\hline TSX-15 & 62 & 60.7 \\
\hline TSX-17 & 43 & 34.3 \\
\hline
\end{tabular}

VII. CONCLUSION

A signal-based characterization of the sea/ocean returns im- 734 aged by TSX sensor has been carried out exploiting 17 data 735 sets, both dual- and quad-pol. The transversal study copes 736 with radiometric, statistical, and polarimetric analysis of the 737 experimental data. One of the goals of this paper is to provide 738 additional insights into proper modeling and characterization of 739 the sea clutter response when observed by TSX.

740

First, the different results indicate that, for the quad-polarized 741 data over maritime scenarios, the thermal noise impact can be 742 a limiting factor in the correct interpretation of the TSX data, 743 particularly for the low sea backscattering regions (incidence 744 angles above $36^{\circ}$ ). Second, taking into account the specific 745 DRA configuration to acquire quad-pol data, the induced tem- 746 poral decorrelation of the sea returns between the polarimetric 747 channels (due to the along-track configuration) is an additional 748 limiting factor that should be carefully considered. This effect, 749 combined with the additional thermal decorrelation, needs to be 750 properly accounted for when trying to fit polarimetric physical- 751 based models to the experimental data, in order to avoid mis- 752 interpretation. In this line, this paper proposes a new modeling 753 approach to include such system/scenario dependent limitations 754 applied specifically to the X-Bragg model and concreted in the 755 new X2-Bragg model.

756

The NRCS analysis shows that the semiempirical model 757 XMOD2 provides a fair fitting to the measured $\sigma^{0}$. Neverthe- 758 less, further studies are required to obtain a refined tunning of 759 the XMOD2 coefficients from quad-pol TSX data sets for a 760 wide range of sea conditions, ensuring that adequate calibration 761 of the DRA mode can be accomplished.

Deviation from the Gaussian statistics for the TSX sea data 763 is observed, when sufficient SNR is available and for small 764 to medium incidence angles (below 36 ${ }^{\circ}$ ). This means spikier 765 returns and thus heavy-tailed amplitude distributions. The log- 766 cumulant analysis shows that, for TSX, the ocean has high 767 texture due to the higher resolution of the sensor coupled with 768 the higher operating frequency (higher sensitivity to surface 769 roughness variation). These results are in line with the study 770 of the PR, which indicates an important contribution of non- 771 Bragg scattering (depolarization), particularly for small inci- 772 dence angles. As indicated in [15], [28], and [29], the presence 773 of breaking wave events, increasing surface roughness and 774 producing spikier returns, can explain this high depolarized 775 
776 contribution. These nonpolarized returns tend to reduce as the 777 incidence angle increases.

778 Exploiting the fully polarimetric information through the $779 H / \bar{\alpha}$ plane indicates that, for small incidence angles, the 780 dominant scattering mechanism corresponds to low to medium 781 random surface. The proposed X2-Bragg model proves a quite 782 good fitting to the data in the $H / \bar{\alpha}$ plane. Therefore, the type 783 of average mechanism and the relative strength between the 784 involved scattering mechanisms can be well described by this 785 model. In fact, since the X2-Bragg model, like the original $786 \mathrm{X}$-Bragg, accounts for depolarization in the surface scattering, 787 it is able to properly describe the presence of nonpolarized 788 returns through the $\beta_{1}$ parameter (roughness measure). The 789 fitting of the X2-Bragg to the measured PR indicates that there 790 exists an important contribution of nonpolarized mechanisms 791 ( $\beta_{1}$ values are different from zero, i.e., pure Bragg). Compara792 tively, the X-Bragg model tends to produce an overestimation 793 of the roughness $\left(\beta_{1}\right)$ mainly due to the noise contribution. It 794 has been also recognized that the fully polarimetric data can be 795 exploited to apply an additive NF; otherwise, an overestimation 796 of the PR and thus the roughness for both models is expected. 797 In summary, the study here presented shows that experimen798 tal quad-pol TSX acquisitions over the ocean have intrinsic 799 system/scenario limitations (noise and temporal decorrelation), 800 which should be carefully analyzed to avoid an erroneous inter801 pretation of the polarimetric data. This paper sets the theoretical 802 and analytical foundations to extrapolate the inclusion of such 803 impacts to other physical-based scattering models, evaluating 804 their effectiveness with experimental quad-pol data from cur805 rent state-of-the-art polarimetric SAR sensors.

806

807

808 In the following lines, an extension of the X-Bragg model, 809 referred as X2-Bragg, is presented to introduce the impact of 810 both noise and additional temporal decorrelations, in a two-step 811 procedure.

812 First of all, let us define an equivalent coherency matrix, 813 which accounts for the noise perturbation as (analogous to the 814 study in [19])

$$
\mathbf{T}^{\prime}=\mathbf{T}+\mathbf{N}
$$

815 where $\mathbf{T}$ refers to the expected coherency matrix related to 816 the sea clutter, and the noise coherency matrix is diagonal $817 \mathbf{N}=\sigma_{n}^{2} \mathbf{I}_{3 \times 3}$. For simplicity, it has been assumed that the dif818 ferent polarimetric channels have uncorrelated additive circular 819 complex Gaussian noise with the same power $\sigma_{n}^{2}$.

820 From the previous considerations, and using a general for821 mulation, (8) can be expressed as

$$
\mathbf{T}^{\prime}=\left(\sigma_{1}^{2}+\sigma_{n}^{2}\right) \overline{\mathbf{T}}^{\prime}
$$

822 where the normalized (to $\mathbf{T}_{11}^{\prime}$ ) coherency matrix $\overline{\mathbf{T}}^{\prime}$ is written as

$$
\overline{\mathbf{T}}^{\prime}=\left[\begin{array}{ccc}
1 & \sqrt{\frac{\sigma_{2}^{2}}{\sigma_{1}^{2}} \overbrace{\rho_{\mathrm{SNR}} \rho_{12}}^{\rho_{T}}} & 0 \\
\sqrt{\frac{\sigma_{2}^{2}}{\sigma_{1}^{2}}} \rho_{\mathrm{SNR}_{1}} \rho_{12}^{*} & \frac{\sigma_{2}^{2}}{\sigma_{1}^{2}} \frac{1+1 / \mathrm{SNR}_{2}}{1+1 / \mathrm{SNR}_{1}} & 0 \\
0 & 0 & \frac{\sigma_{3}^{2}}{\sigma_{1}^{2}} \frac{1+1 / \mathrm{SNR}_{3}}{1+1 / \mathrm{SNR}_{1}}
\end{array}\right]
$$

where $\sigma_{1}^{2}, \sigma_{2}^{2}$, and $\sigma_{3}^{2}$ refer to the signal power of the three 823 polarimetric channels (in the Pauli base); $\mathrm{SNR}_{1}, \mathrm{SNR}_{2}$, and 824 $\mathrm{SNR}_{3}$ are the corresponding SNRs, i.e., $\mathrm{SNR}_{i}=\sigma_{i}^{2} / \sigma_{n}^{2} ; \rho_{12} 825$ represents the polarimetric correlation (coherence) coefficient 826 between $\left(S_{\mathrm{HH}}+S_{\mathrm{VV}}\right)$ and $\left(S_{\mathrm{HH}}-S_{\mathrm{VV}}\right)$ channels; and $\rho_{\mathrm{SNR}_{1}} 827$ models the decorrelation effect induced by the presence of 828 thermal noise, i.e.,

$$
\rho_{\mathrm{SNR}_{1}}=\frac{1}{1+1 / \mathrm{SNR}_{1}}
$$

such that the total coherence, based on a multiplicative model, 830 is defined as $\rho_{T}$.

From the original X-Bragg model formulation in (7), the 832 different parameters in (10) can be expressed as

$$
\begin{aligned}
\sigma_{1}^{2} & =m_{s}^{2} C_{1} \\
\sigma_{2}^{2} & =m_{s}^{2} C_{3}\left(1+\operatorname{sinc}\left(4 \beta_{1}\right)\right) \\
\sigma_{3}^{2} & =m_{s}^{2} C_{3}\left(1-\operatorname{sinc}\left(4 \beta_{1}\right)\right) \\
\rho_{12} & =\frac{C_{2} \operatorname{sinc}\left(2 \beta_{1}\right)}{\sqrt{C_{1} C_{3}\left(1+\operatorname{sinc}\left(4 \beta_{1}\right)\right)}} .
\end{aligned}
$$

From the previous relationships, the three polarimetric SNRs 834 can be defined in terms of an equivalent $\mathrm{SNR}_{\mathrm{Eq}}$ as

$$
\begin{aligned}
& \mathrm{SNR}_{1}=C_{1} \mathrm{SNR}_{\mathrm{Eq}} \\
& \mathrm{SNR}_{2}=C_{3}\left(1+\operatorname{sinc}\left(4 \beta_{1}\right)\right) \mathrm{SNR}_{\mathrm{Eq}} \\
& \mathrm{SNR}_{3}=C_{3}\left(1-\operatorname{sinc}\left(4 \beta_{1}\right)\right) \mathrm{SNR}_{\mathrm{Eq}}
\end{aligned}
$$

in a way that, if $\mathrm{SNR}_{1}$ is estimated from the data, the equivalent 836 $\mathrm{SNR}_{\mathrm{Eq}}=m_{s}^{2} / \sigma_{n}^{2}$ can be inverted from the model and used to 837 properly account for the thermal noise impact in the X-Bragg 838 model fitting.

839

In multichannel adaptive array and GMTI theory, the impact 840 of temporal decorrelation is known to cause an increase in 841 the number of eigenvalues (of the clutter-plus-noise covariance 842 matrix) different from the noise floor [3]. The X-Bragg model 843 has been further extended to account for temporal decorrelation, 844 in terms of an additional coherence coefficient $\rho_{\text {temp }}$. It must 845 be noted that this term cannot be directly introduced in matrix 846 (10) since, from the DRA acquisition itself, the temporal decor- 847 relation is between the individual polarimetric channels with 848 some spatial or temporal baseline. This additional term should 849 be considered in the covariance matrix $\mathbf{C}$ formulation. First, a 850 transformation of the normalized coherency matrix $\overline{\mathbf{T}}^{\prime}$ (10) into 851 the covariance matrix is performed as

$$
\overline{\mathbf{C}}^{\prime}=\left\{\mathbf{U}_{3(\mathrm{~L} \rightarrow \mathrm{P})}\right\}^{-1} \overline{\mathbf{T}}^{\prime}\left\{\mathbf{U}_{3(\mathrm{~L} \rightarrow \mathrm{P})}^{H}\right\}^{-1}
$$

where the unitary transformation $(\mathrm{L} \rightarrow \mathrm{P})$ from the lexico- 853 graphic target vector to the Pauli vector is

$$
\mathbf{U}_{3(\mathrm{~L} \rightarrow \mathrm{P})}=\frac{1}{\sqrt{2}}\left[\begin{array}{ccc}
1 & 0 & 1 \\
1 & 0 & -1 \\
0 & \sqrt{2} & 0
\end{array}\right] .
$$

Then, the additional coherence coefficient $\rho_{\text {temp }}$ is included 855 as multiplicative term into the off-diagonal terms of $\overline{\mathbf{C}}^{\prime}$ to obtain 856 $\overline{\mathbf{C}}^{\prime \prime}$. The normalized coherency matrix for the X2-Bragg model 857 


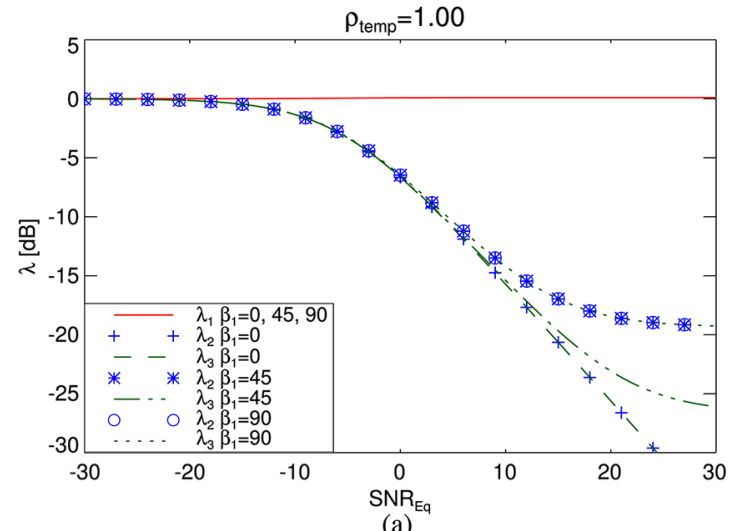

(a)

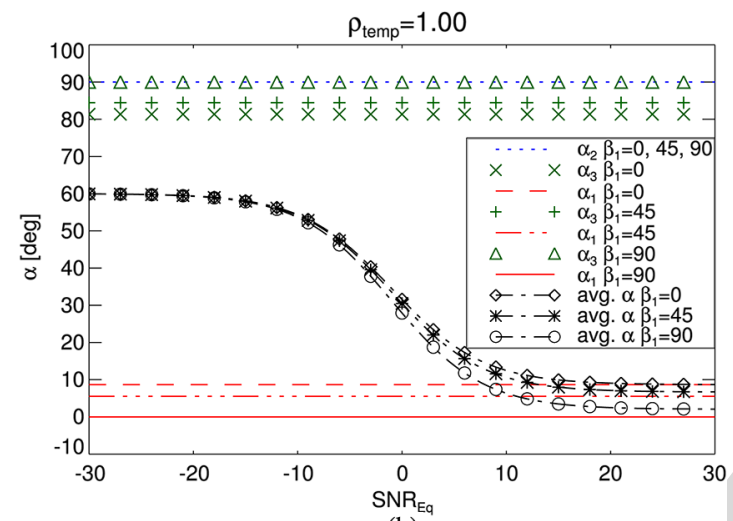

(b)

Fig. 11. Sensitivity w.r.t. $\mathrm{SNR}_{\mathrm{Eq}}$ for the X2-Bragg model considering the noise impact and a temporal decorrelation $\rho_{\text {temp }}=1.0$ for three values of the uniform distribution width $\beta_{1}$; an $\mathrm{X}$-band system $(9.65 \mathrm{GHz})$, an incidence angle $\gamma=24.6^{\circ}$, a sea temperature of $10^{\circ} \mathrm{C}$, and salinity of $35 \%$ are considered. (a) Eigenvalues. (b) Alphas.

$858 \overline{\mathbf{T}}^{\prime \prime}$ can be obtained using a matrix transformation of $\overline{\mathbf{C}}^{\prime \prime}$ by 859 means of $\mathrm{U}_{3(\mathrm{~L} \rightarrow \mathrm{P})}$, inversely analogous to the case in (14).

860

861

862 The sensitivity of the eigenvalues and $\alpha$ parameters as a 863 function of $\mathrm{SNR}_{\mathrm{Eq}}$ for $\rho_{\mathrm{temp}}=1.0$ and $\rho_{\text {temp }}=0.95$ is shown, 864 respectively, in Figs. 11 and 12, exploiting the theoretical 865 coherency matrix formulation $\overline{\mathbf{T}}^{\prime \prime}$ of the X2-Bragg model. It 866 has been assumed an X-band system $(9.65 \mathrm{GHz})$, an incidence 867 angle $\gamma$ of $24.6^{\circ}$ [corresponding to the center of TSX-4 acquisi868 tion (see Table I)], and sea conditions of $10^{\circ} \mathrm{C}$ and $35 \%$ salinity 869 content. In Figs. 11(a) and 12(a), the eigenvalue distribution 870 is represented as a function of $\mathrm{SNR}_{\mathrm{Eq}}$. The three eigenvalues 871 collapse, regardless of $\beta_{1}$ and $\rho_{\text {temp }}$, when reducing $\mathrm{SNR}_{\mathrm{Eq}}$ $872(<-10 \mathrm{~dB})$, being the noise the dominant mechanism.

873 In case of $\beta_{1}=0^{\circ}$ (smooth surface) and with increasing $874 \mathrm{SNR}_{\mathrm{Eq}}$, the two smallest eigenvalues $\lambda_{2}$ (plus symbols) and $\lambda_{3}$ 875 (dashed line) have the same decreasing trend when no temporal 876 decorrelation is included [see Fig. 11(a)]. Their contribution is $87730 \mathrm{~dB}$ below $\lambda_{1}$ for $\mathrm{SNR}_{\mathrm{Eq}}>20 \mathrm{~dB}$, in which case SPM ap878 plies with a single dominant mechanism. This can be observed 879 also from the averaged value $\bar{\alpha}$ [dash-dot line with diamond 880 markers in Fig. 11(b)], which tends to $\alpha_{1}$ (dashed line). As $\beta_{1}$
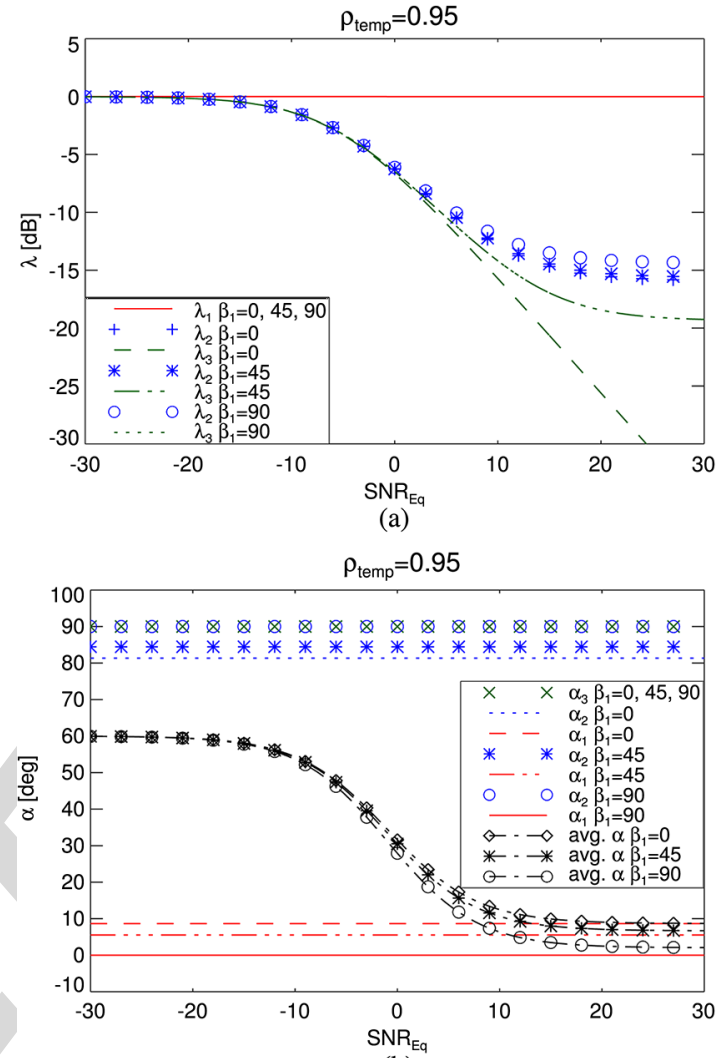

(b)

Fig. 12. Sensitivity w.r.t. $\mathrm{SNR}_{\mathrm{Eq}}$ for the X2-Bragg model considering the noise impact and a temporal decorrelation $\rho_{\text {temp }}=0.95$ for three values of the uniform distribution width $\beta_{1}$; an X-band system $(9.65 \mathrm{GHz})$, an incidence angle $\gamma=24.6^{\circ}$, a sea temperature of $10^{\circ} \mathrm{C}$, and salinity of $35 \%$ are considered. (a) Eigenvalues. (b) Alphas.

(roughness) increases, the cross-polar power increases; hence, 881 more than a single dominant mechanism exist. For $\beta_{1}=45^{\circ} 882$ and $\mathrm{SNR}_{\mathrm{Eq}}>10 \mathrm{~dB}$, the two smallest eigenvalues (asterisk 883 symbols and dash-dot-dot line) start to diverge. In the limit 884 $\beta_{1}=90^{\circ}$ and with no additional temporal decorrelation, $\lambda_{2} 885$ (circle markers) and $\lambda_{3}$ (dotted line) have the same trend as a 886 function of the effective SNR, since polarimetric coherence $\rho_{12} 887$ decreases to 0 . Considering a temporal decorrelation of the sea 888 $\rho_{\text {temp }}=0.95$, a general increase of the second eigenvalue can 889 be recognized (for $\mathrm{SNR}_{\mathrm{Eq}}>10 \mathrm{~dB}$ ), comparing Figs. 11(a) and 890 12(a), particularly for $\beta_{1}=0^{\circ}$. In case of $\lambda_{3}$, this behavior is 891 also observed, but for $\beta_{1} \geq 45^{\circ}$.

Contrary to the eigenvalues, the individual $\alpha$ parameters 893 are almost unaffected by the presence of noise, as shown in 894 Figs. 11(b) and 12(b). Polarimetric decomposition considers 895 the mean $\bar{\alpha}$, based on proper weighting of the individual $\alpha 896$ terms through the eigenvalues' probabilities [14]. As shown in 897 Figs. 11(b) and 12(b), the average $\bar{\alpha}$ presents a large variation 898 as a function of $\mathrm{SNR}_{\mathrm{Eq}}$ for different values of $\beta_{1}$. In the limit, 899 $\bar{\alpha}$ tends to $60^{\circ}$, such that the scattering mechanism lies in the 900 extreme edge of region 2 of the Cloude-Pottier $H / \bar{\alpha}$, i.e., 901 random noise, that is, no polarization dependence. Temporal 902 decorrelation exchanges the behavior of the individual $\alpha_{2}$ and 903 $\alpha_{3}$ parameters, comparing Figs. 11(b) and 12(b), but does not 904 affect the averaged values: when no temporal decorrelation 905 


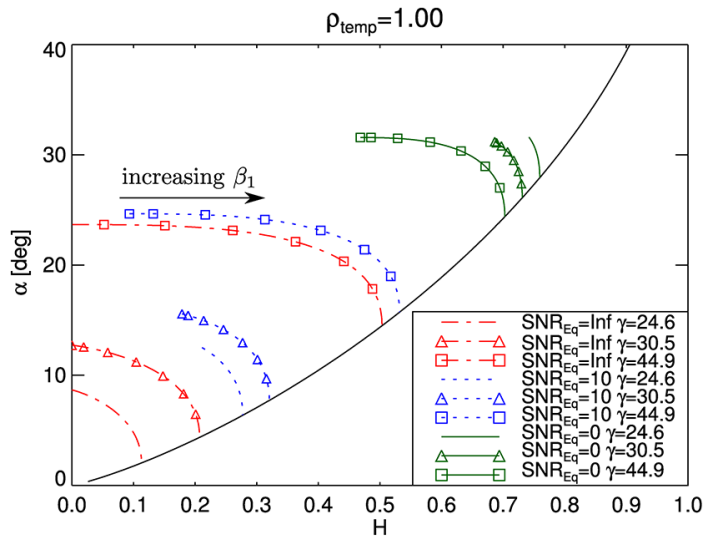

(a)

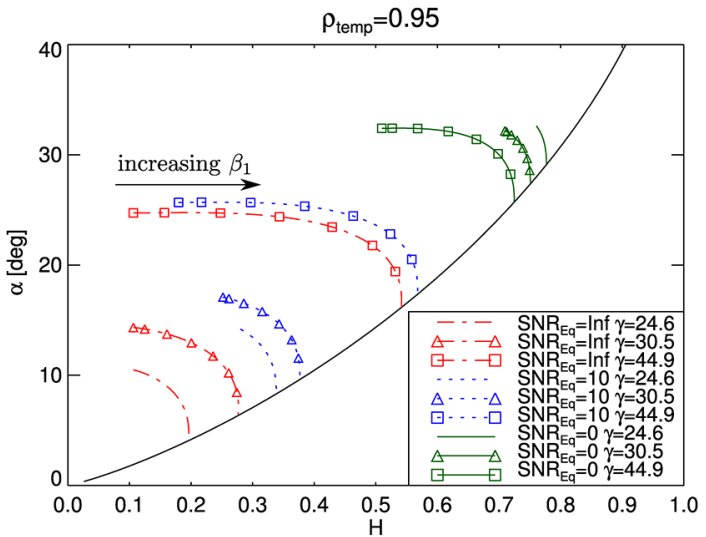

(b)

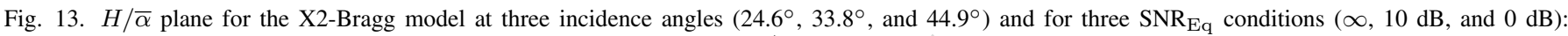
(a) $\rho_{\text {temp }}=1.0$ and (b) $\rho_{\text {temp }}=0.95$ (sea water with salinity content of $35 \%$ and temperature of $10^{\circ} \mathrm{C}$ ).

906 is considered $\left(\rho_{\text {temp }}=1\right), \alpha_{2}$ collapses to $90^{\circ}$ regardless of $907 \beta_{1}$, and $\alpha_{3}$ sweeps the values from $80^{\circ}$ to $90^{\circ}$, whereas for $908 \rho_{\text {temp }}=0.95, \alpha_{3}$ is $90^{\circ}$ for the different roughness parameters. 909 Therefore, temporal decorrelation is not impairing the values 910 of $\alpha$ parameters, but it is modulating the contribution of the dif911 ferent mechanisms, i.e., for $\rho_{\text {temp }}=0.95$, the cross-polar com912 ponent (related to $\alpha=90$ independently from $\beta_{1}$ ) is no longer 913 the second dominant scattering mechanism (as for $\left.\rho_{\text {temp }}=1\right)$. 914 The impact of both thermal noise and temporal decorre915 lation in the X2-Bragg model is also analyzed in the $H / \bar{\alpha}$ 916 plane, as represented in Fig. 13(a) and (b) for $\rho_{\text {temp }}=1$ and $917 \rho_{\text {temp }}=0.95$, respectively. Different incidence angles, corre918 sponding to the center of data takes TSX-4 $\left(24.6^{\circ}\right)$, TSX-7 $919\left(30.5^{\circ}\right)$, and TSX-17 $\left(44.9^{\circ}\right)$, are considered. The noise impact 920 is translated into a progressive increase of both $\bar{\alpha}$ and $H$ as $921 \mathrm{SNR}_{\mathrm{Eq}}$ decreases. Moreover, the variation of the $H / \bar{\alpha}$ pairs as a 922 function of $\beta_{1}$ is reduced when the noise contribution increases. 923 Temporal decorrelation produces similar effects, but its impact 924 reduces as long as the entropy increases, clearly visible for the 925 region with $H>0.6$ when comparing Fig. 13(a) and (b).

926 A particular case in the $H / \bar{\alpha}$ plane is recognized for $927 \mathrm{SNR}_{\mathrm{Eq}}=0 \mathrm{~dB}$, where the variation as a function of the in928 cidence angle is reversed, i.e., the near range presents higher 929 entropy values than the far range: as long as the noise con930 tribution increases, the divergence between the two smallest 931 eigenvalues $\left(\lambda_{2}\right.$ and $\left.\lambda_{3}\right)$ reduces (increasing the entropy), and 932 this effect is more severe for the near range. This relative sepa933 ration is also a function of the roughness $\left(\beta_{1}\right)$, which, for noisy 934 near range scenarios, is quite flat. Such a response justifies 935 the reduction on $H$ as a function of $\beta_{1}$ for increasing noise, 936 particularly at near range.

\section{ACKNOWLEDGMENT}

938 The authors would like to thank the German Aerospace 939 Center (DLR) and, in particular, S. Baumgartner for the TSX 940 quad-pol data provided in the frame of the DRA proposal 941 MTH1971 and the FP7-SPACE Project NEREIDS Ref. 263468 942 for the TSX dual-pol data. The authors would also like to thank 943 A. Gómez for her helpful suggestions and comments.

\section{REFERENCES}

[1] S. Hasselmann, C. Brüning, K. Hasselmann, and P. Heimbach, “An im- 945 proved algorithm for the retrieval of ocean wave spectra from synthetic 946 aperture radar image spectra," J. Geophys. Res., Oceans, vol. 101, no. C7, 947 pp. 16615-16629, Jul. 1996.

[2] R. Goldstein and H. Zebker, "Interferometric radar measurement of ocean 949 surface currents," Nature, vol. 328, no. 6132, pp. 707-709, Aug. 1987.950

[3] E. Makhoul, A. Broquetas, J. Ruiz-Rodon, Y. Zhan, and F. Ceba, 951 "A performance evaluation of SAR-GMTI missions for maritime applica- 952 tions," IEEE Trans. Geosci. Remote Sens., vol. 53, no. 5, pp. 2496-2509, 953 May 2015.

[4] M. Gabele et al., "Fore and aft channel reconstruction in the TerraSAR-X 955 dual receive antenna mode," IEEE Trans. Geosci. Remote Sens., vol. 48, 956 no. 2, pp. 795-806, Feb. 2010.

[5] I. Hajnsek, E. Pottier, and S. Cloude, "Inversion of surface parameters 958 from polarimetric SAR," IEEE Trans. Geosci. Remote Sens., vol. 41, 959 no. 4, pp. 727-744, Apr. 2003.

960

[6] X.-M. Li and S. Lehner, "Algorithm for sea surface wind retrieval from 961 TerraSAR-X and TanDEM-X data," IEEE Trans. Geosci. Remote Sens., 962 vol. 52, no. 5, pp. 2928-2939, May 2014.

[7] T. Fritz and M. Eineder, "TerraSAR-X: Ground segment basic product 964 specification document," German Aerosp. Center (DLR), Köln, Germany, 965 Tech. Rep. TX-GS-DD-3302, Oct. 2013.

[8] K. D. Ward, R. J. Tough, and S. Watts, Sea Clutter: Scattering, the K 967 Distribution and Radar Performance. Stevenage, U.K.: The Insti. Eng. 968 Technol., 2006.

[9] S. Anfinsen and T. Eltoft, "Application of the matrix-variate Mellin trans- 970 form to analysis of polarimetric radar images," IEEE Trans. Geosci. 971 Remote Sens., vol. 49, no. 6, pp. 2281-2295, Jun. 2011.

[10] S. Skrunes, C. Brekke, T. Eltoft, and V. Kudryavtsev, "Comparing 973 near-coincident C- and X-band SAR acquisitions of marine oil spills," 974 IEEE Trans. Geosci. Remote Sens., vol. 53, no. 4, pp. 1958-1975, 975 Apr. 2015.

[11] E. Jakeman and P. N. Pusey, “A model for non-Rayleigh sea echo," IEEE 977 Trans. Antennas Propag., vol. AP-24, no. 6, pp. 806-814, Nov. 1976.99

[12] K. Ouchi and R. Cordey, "Statistical analysis of azimuth streaks observed 979 in digitally processed CASSIE imagery of the sea surface," IEEE Trans. 980 Geosci. Remote Sens., vol. 29, no. 5, pp. 727-735, Sep. 1991.

[13] D. Trizna, "Statistics of low grazing angle radar sea scatter for moder- 982 ate and fully developed ocean waves," IEEE Trans. Antennas Propag., 983 vol. 39, no. 12, pp. 1681-1690, Dec. 1991.

[14] S. Cloude and E. Pottier, "An entropy based classification scheme for land 985 applications of polarimetric SAR,” IEEE Trans. Geosci. Remote Sens., 986 vol. 35, no. 1, pp. 68-78, Jan. 1997.

[15] V. Kudryavtsev, D. Hauser, G. Caudal, and B. Chapron, "A semiempirical 988 model of the normalized radar cross-section of the sea surface 1. Back- 989 ground model," J. Geophys. Res., Oceans, vol. 108, no. C3, pp. FET 2-1- 990 FET 2-24, Mar. 2003.

[16] J. Morris, S. Anderson, and S. Cloude, "A study of the X-band entropy 992 of breaking ocean waves," in Proc. IEEE IGARSS, Jul. 2003, vol. 2, 993 pp. 711-713.

[17] D. A. Gagliardini and P. C. Colón, "Ocean feature detection using mi- 995 crowave backscatter and sun glint observations," Gayana (Concepción), 996 vol. 68 , no. 2 , pp. $180-185,2004$. 
998 [18] AIRBUS, "Radiometric calibration of TerraSAR-X data: Beta naught and 999 sigma naught coefficient calculation,” AIRBUS Defence Space, Munich, 1000 Germany, Tech. Rep. TSXX-ITD-TN-0049, Mar. 2014

1001 [19] C. López-Martínez, A. Alonso-González, and X. Fabregas, "Perturbation 1002 analysis of eigenvector-based target decomposition theorems in radar po1003 larimetry," IEEE Trans. Geosci. Remote Sens., vol. 52, no. 4, pp. 20811004 2095, Apr. 2014.

1005 [20] V. Gregers-Hansen and R. Mital, "An improved empirical model for radar 1006 sea clutter reflectivity,” IEEE Trans. Aerosp. Electron. Syst., vol. 48, no. 4, 1007 pp. 3512-3524, Oct. 2012.

1008 [21] F. E. Nathanson, J. P. Reilly, and M. N. Cohen, Radar Design Principles: 1009 Signal Processing and the Environment, 2nd ed. New York, NY, USA: 1010 McGraw-Hill, 1990.

1011 [22] M. Menon, "Estimation of the shape and scale parameters of the Weibull 1012 distribution," Technometrics, vol. 5, no. 2, pp. 175-182, May 1963.

1013 [23] D. Iskander and A. Zoubir, "Estimation of the parameters of the 1014 K-distribution using higher order and fractional moments [radar clut1015 ter]," IEEE Trans. Aerosp. Electron. Syst., vol. 35, no. 4, pp. 1453-1457, 1016 Oct. 1999.

1017 [24] D. Blacknell and R. J. A. Tough, "Parameter estimation for the 1018 K-distribution based on $[\mathrm{z} \log (\mathrm{z})]$," Proc. Inst. Elect. Eng.-Radar Sonar 1019 Navig., vol. 148, no. 6, pp. 309-312, Dec. 2001.

1020 [25] R. J. A. Tough, D. Blacknell, and S. Quegan, "A statistical description 1021 of polarimetric and interferometric synthetic aperture radar data," Proc. 1022 R. Soc. Lond. A, Math. Phys. Sci., vol. 449, no. 1937, pp. 567-589, 1023 Jun. 1995

1024 [26] D. Schuler and J. Lee, "Mapping ocean surface features using bio1025 genic slick-fields and SAR polarimetric decomposition techniques," Proc. 1026 Inst. Elect. Eng.-Radar Sonar Navig., vol. 153, no. 3, pp. 260-270, 1027 Jun. 2006.

1028 [27] M. Migliaccio, A. Gambardella, and M. Tranfaglia, "SAR polarimetry 1029 to observe oil spills," IEEE Trans. Geosci. Remote Sens., vol. 45, no. 2, 1030 pp. 506-511, Feb. 2007.

1031 [28] A. Voronovich and V. Zavorotny, "Theoretical model for scattering of 1032 radar signals in Ku-and C-bands from a rough sea surface with breaking 1033 waves," Waves Random Media, vol. 11, no. 3, pp. 247-269, 2001.

1034 [29] A. A. Mouche, D. Hauser, and V. Kudryavtsev, "Radar scattering of the 1035 ocean surface and sea-roughness properties: A combined analysis from 1036 dual-polarizations airborne radar observations and models in C band," 1037 J. Geophys. Res., Oceans, vol. 111, no. C9, Sep. 2006, Art. ID. C09004.

1038 [30] D. Schuler, J.-S. Lee, and K. Hoppel, "Polarimetric SAR image signatures 1039 of the ocean and gulf stream features," IEEE Trans. Geosci. Remote Sens., 1040 vol. 31, no. 6, pp. 1210-1221, Nov. 1993.

1041 [31] L. Klein and C. Swift, "An improved model for the dielectric constant 1042 of sea water at microwave frequencies," IEEE Trans. Antennas Propag., 1043 vol. AP-25, no. 1, pp. 104-111, Jan. 1977.

1044 [32] R. Romeiser and D. Thompson, "Numerical study on the along-track 1045 interferometric radar imaging mechanism of oceanic surface currents," 1046 IEEE Trans. Geosci. Remote Sens., vol. 38, no. 1, pp. 446-458, Jan. 2000.

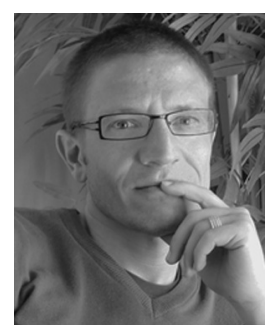

Carlos López-Martínez (S'97-M'04-SM'11) re- 1069 ceived the M.Sc. degree in electrical engineering 1070 and the Ph.D. degree from Universitat Politècnica 1071 de Catalunya (UPC), Barcelona, Spain, in 1999 and 1072 2003, respectively.

1073

From October 2000 to March 2002, he was 1074 with the Frequency and Radar Systems Depart- 1075 ment, HR, German Aerospace Center (DLR), 1076 Oberpfaffenhofen, Germany. From June 2003 to De- 1077 cember 2005, he was with the Image and Remote 1078 Sensing Group SAPHIR Team of the Institute of 1079 Electronics and Telecommunications of Rennes (IETR CNRS UMR 6164), 1080 Rennes, France. In January 2006, he joined UPC, Barcelona, as a Ramón 1081 y Cajal Researcher. He is currently an Associate Professor in the area of 1082 remote sensing and microwave technology with UPC. He has authored or 1083 coauthored over 100 articles in journals, books, and conference proceedings 1084 in the radar remote sensing and image analysis literature. His research interests 1085 include SAR and multidimensional SAR, radar polarimetry, physical parameter 1086 inversion, digital signal processing, estimation theory, and harmonic analysis. 1087

Dr. López-Martínez is an Associate Editor of the IEEE JOURNAL OF SE- 1088 LeCted Topics in ApPlied Earth ObServations and Remote SENS- 1089 ING. He served as a Guest Editor of the EURASIP Journal on Advances in 1090 Signal Processing. He has organized different invited sessions in international 1091 conferences on radar and SAR polarimetry. He has presented advanced courses 1092 and seminars on radar polarimetry to a wide range of organizations and events. 1093 He was a recipient of the Student Prize Paper Award at the EUSAR 20021094 Conference and coauthored the paper awarded with the First Place Student 1095 Paper Award at the EUSAR 2012 Conference. He has been also a recipient of 1096 the IEEE Geoscience and Remote Sensing Society 2013 GOLD Early Career 1097 Award.

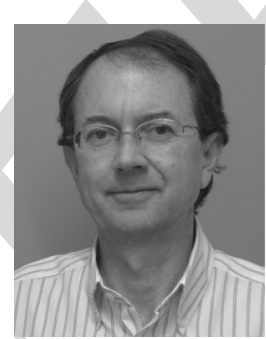

Antoni Broquetas (S'84-M'90) was born in 1099 Barcelona, Spain, in 1959. He received the Ingeniero 1100 degree in telecommunications engineering and the 1101 Doctor Ingeniero degree in telecommunications en- 1102 gineering (for his work on microwave tomography) 1103 from Universitat Politècnica de Catalunya (UPC), 1104 Barcelona, in 1985 and 1989, respectively. $\quad 1105$

In 1986, he was a Research Assistant with 1106 Portsmouth Polytechnic, Portsmouth, U.K., involved 1107 in propagation studies. In 1987, he joined the Depart- 1108 ment of Signal Theory and Communications (TSC), 1109 UPC, where he was the Subdirector of Research of the Institute of Geomatics 1110 from 1998 to 2002 and the Director of TSC from 2003 to 2006. Since 1111 1999, he has been a Full Professor with UPC, involved in research on radar 1112 imaging and remote sensing. He has authored or coauthored over 150 papers 1113 on microwave tomography, radar, ISAR and SAR systems, and SAR processing 1114 and interferometry.

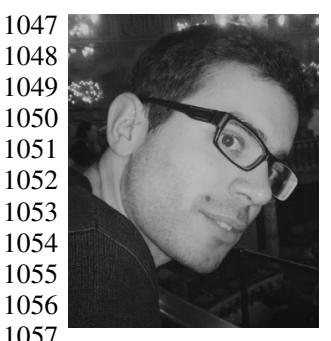

Eduardo Makhoul (S'09-M'15) was born in Homs, Siria, in 1985. He received the M.Sc. degree in electrical engineering and the Ph.D. degree (cum laude) from Universitat Politècnica de Catalunya (UPC), Barcelona, Spain, in 2009 and 2015, respectively.

In 2008, he worked on his master thesis in the frame of high-resolution wide-swath synthetic aperture radar (SAR) systems at the German Aerospace Center (DLR). In 2009, he joined the Remote Sensing Laboratory, UPC, researching on aspects of ground moving target indication (GMTI) for future 1058 spaceborne SAR systems. From 2009 to 2011, he worked on the radiomet1059 ric budget analysis of the Spanish mission PAZ. From 2010 to 2013, he 1060 worked on the analysis and study of SAR-GMTI systems and techniques for 1061 future European missions in the frame of NEWA and SIMTISYS projects. In 10622013 (April-August), he was a Visiting Researcher with DLR, working on 1063 SAR-GMTI processing of airborne and spaceborne data over maritime scenar1064 ios. Since August 2015, he has been with IsardSAT, an experienced Earth obser1065 vation research company in Barcelona, involved in the design, calibration, and 1066 maintenance of civil remote sensing instruments. His main research interests 1067 include SAR, GMTI, radar system design, mission performance, calibration, 1068 array signal processing, sea/ocean surveillance, and polarimetry.

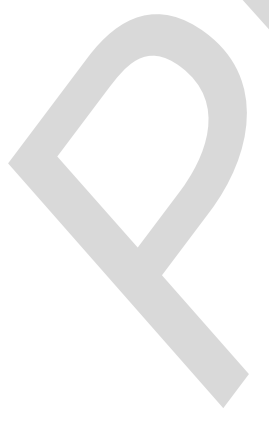




\section{AUTHOR QUERIES}

\section{AUTHOR PLEASE ANSWER ALL QUERIES}

Please be aware that authors are required to pay overlength page charges (\$200 per page) if the paper is longer than 6 pages. If you cannot pay any or all of these charges please let us know.

This pdf contains 2 proofs. The first half is the version that will appear on Xplore. The second half is the version that will appear in print. If you have any figures to print in color, they will be in color in both proofs.

The "Open Access" option for your paper expires when the paper is published on Xplore in an issue with page numbers. Papers in "Early Access" may be changed to "Open Access."

AQ1 = Please check if changes made in the affiliation of author E. Makhoul are correct. Otherwise, please make the necessary changes.

AQ2 = Please provide the expanded form of "DFO."

END OF ALL QUERIES 


\title{
Exploiting Polarimetric TerraSAR-X Data for Sea Clutter Characterization
}

3

\author{
Eduardo Makhoul, Member, IEEE, Carlos López-Martínez, Senior Member, IEEE, and
}

Antoni Broquetas, Member, IEEE

\begin{abstract}
5 Abstract-This paper presents a detailed characterization of 6 sea/ocean clutter returns at X-band, imaged by TerraSAR-X 7 (TSX) mission from the radiometric, statistical, and polarimet8 ric standpoints. Different TSX data takes, covering the typical 9 spaceborne incidence angle region $\left(20^{\circ}-\mathbf{4 5}^{\circ}\right)$, are analyzed: dual10 polarized and experimental quad-polarized (quad-pol) data have 11 been used. The thermal noise of the receiver for quad-pol data 12 turns out to be an important limitation in the sea characterization 13 from TSX, particularly at high incidence angles (above $36^{\circ}$ ), where 14 low signal-to-noise ratios (SNRs) can impair the proper data 15 distribution fitting and the polarimetric backscattering descrip16 tion. Statistical analysis shows large deviation from Gaussianity, 17 indicating presence of texture, mainly in the small incidence region $18\left(\mathbf{2 0}^{\circ}-\mathbf{3 6}^{\circ}\right)$, with favorable SNR conditions. The different polari19 metric features revealed contribution of nonpolarized scattering, 20 related to the presence of breaking waves. This paper also proposes 21 and evaluates an extension of the well-known X-Bragg model, 22 named X2-Bragg (extended-extended Bragg), which properly ac23 counts for the impact of thermal noise and sea clutter temporal 24 decorrelation, due to the dual receive antenna acquisition mode. 25 Such additional decorrelation sources, if not properly analyzed 26 and accounted for in the physical-based model description, could 27 lead to an incorrect interpretation of the polarimetric properties 28 and the related erroneous geophysical parametric inversion from 29 the real data. In this sense, the X2-Bragg proves its fitting to 30 the experimental data, quantifying accordingly the presence of 31 nonpolarized scattering in terms of the roughness parameter $\beta_{1}$.
\end{abstract}

32 Index Terms-Non-Gaussian clutter, parameter estimation, 33 radar backscattering coefficient, sea clutter, statistical analysis, 34 synthetic aperture radar (SAR), SAR polarimetry, X-Bragg.

\section{INTRODUCTION}

36 $\Gamma$ HE imaging capability of synthetic aperture radars (SARs), 38 independent from daylight and weather conditions, repre39 sents a potential tool for globally monitoring the ocean. SAR is 40 a very important forecast instrument in oceanography, allowing, 41 among others, the retrieval of ocean wave spectrums [1] and

Manuscript received April 24, 2015; revised July 9, 2015; accepted July 12, 2015. This work was supported in part by the FPU Research Fellowship Program, Ministerio de Educación under Contract AP2009-4590; by the FI-AGAUR Research Fellowship Program, Generalitat de Catalunya under Contract 2010FI EM051757; and by the Spanish Ministry of Science and Innovation (MICINN) under projects TEC2011-28201-C02-01 and TIN201455413-C2-1-P.

E. Makhoul was with the Department of Signal Theory and Communications, Universitat Politècnica de Catalunya, 08034 Barcelona, Spain. He is now with isardSAT, 08042 Barcelona, Spain (e-mail: edumak@tsc.upc.edu).

C. López-Martínez and A. Broquetas are with the Department of Signal Theory and Communications, Universitat Politècnica de Catalunya, 08034 Barcelona, Spain (e-mail: carlos.lopez@tsc.upc.edu; broquetas@tsc.upc.edu).

Color versions of one or more of the figures in this paper are available online at http://ieeexplore.ieee.org.

Digital Object Identifier 10.1109/TGRS.2015.2457242 the measurement of ocean or river current velocities [2]. Lately, 42 the increase in vessel hijacking, as well as the need to control 43 fishing and tanker polluting activities, demand remote sensing 44 systems to properly monitor maritime traffic. SAR sensors with 45 ground moving target indication (GMTI) capabilities are of 46 great interest for ship surveillance [3].

Characterization and modeling of the sea clutter returns 48 observed by SAR systems are of great interest for the accurate 49 evaluation of future SAR missions imaging the sea. The avail- 50 ability of polarimetric SAR instruments, such as TerraSAR-X 51 (TSX) or TanDEM-X (TDX), provides a unique opportunity to 52 gather geophysical and biophysical information of the marine 53 environment. This paper exploits different dual-pol $\left(\mathrm{HH} / \mathrm{HV}^{1}\right) 54$ and experimental quad-pol TSX data sets (covering $20^{\circ}-45^{\circ} 55$ of incidence angle) to perform a transversal (radiometric, sta- 56 tistical, and polarimetric) and compact study of the sea echo 57 returns. To the authors' knowledge, such analysis has not 58 been performed yet, exploiting the experimental quad-pol data 59 from TSX.

The main stress of the study is to point out the different 61 limitations that may be encountered when operating TSX, par- 62 ticularly in the quad-pol mode, where the dual receive antenna 63 (DRA) mode is used [4], such that the antenna is split into two 64 halves (receiving $\mathrm{H}$ and $\mathrm{V}$ simultaneously) and the transmit 65 polarization is toggled in a pulse basis. On one hand, there is 66 an increase of $3 \mathrm{~dB}$ in the noise equivalent sigma zero (NESZ) 67 due to a reduced antenna effective area per channel, i.e., 3-dB 68 loss in signal-to-noise ratio (SNR). On the other hand, a tem- 69 poral lag exists between the different polarimetric channels, 70 during which sea clutter decorrelates. The identification of such 71 technological-driven aspects from the signal-based analysis 72 proves to be of key importance in the correct interpretation of 73 the polarimetric data when fitting physical-based models. In 74 this sense, the X-Bragg model [5], which extends the Bragg 75 surface scattering to include the impact of depolarized and 76 cross-polarized returns, has been optimized to account for such 77 additional perturbations (noise+sea temporal decorrelations), 78 based on a comprehensive theoretical formulation, leading to 79 the extended-extended Bragg (X2-Bragg) model; otherwise, 80 these additional decorrelation sources could produce an erro- 81 neous interpretation of the polarimetric backscattering proper- 82 ties when trying to fit the physical-based X-Bragg model to the 83 experimental data.

The radiometric analysis, in terms of the radar backscattering 85 coefficient $\sigma^{0}$, shows that, in TSX, the thermal noise of the re- 86

${ }^{1} \mathrm{H}$ and $\mathrm{V}$ refer to horizontal and vertical polarizations, respectively. 
TABLE I

TSX Data Takes Used in the Sea Clutter Characterization (BW $R$ and BW $A$ Are Range and Azimuth Processed BANDWIDTHS, $v_{\text {win }}$ AND $\theta_{\text {win }}$ ARE WIND SPEED AND DiRECTION FROM Which THE Wind Is Blowing)

\begin{tabular}{|c|c|c|c|c|c|c|c|c|c|}
\hline ID & Date & Region & Location & Mode & $\gamma[\mathbf{d e g}]$ & $\boldsymbol{B W}_{\boldsymbol{R}}[\mathbf{M H z}]$ & $\boldsymbol{B W}_{\boldsymbol{A}}[\mathbf{H z}]$ & $\boldsymbol{v}_{\boldsymbol{w i n}}[\mathbf{m} / \mathbf{s}]$ & $\boldsymbol{\theta}_{\boldsymbol{w i n}}[\mathbf{d e g}]$ \\
\hline \hline TSX-1 & 05.03 .2013 & Senegal & N15.07-W17.60 & Duad-Pol & $19.85-21.74$ & 150 & 1380 & - & - \\
\hline TSX-2 & 09.03 .2013 & Gulf of Guinea & N3.56-W2.35 & Duad-Pol & $22.43-24.26$ & 150 & 1380 & - & - \\
\hline TSX-3 & 25.04 .2010 & Vancouver Island & N49.72-W127.97 & Quad-Pol & $23.75-25.49$ & 150 & 1380 & 12.1 & 143 \\
\hline TSX-4 & 06.05 .2010 & Vancouver Island & N49.72-W127.97 & Quad-Pol & $23.75-25.49$ & 150 & 1380 & 6.3 & 331 \\
\hline TSX-5 & 16.03 .2013 & Mauritania & N19.45-W16.78 & HH & $24.80-27.99$ & 150 & 2765 & - & - \\
\hline TSX-6 & 30.04 .2010 & Bermuda & N31.37-W69.54 & Quad-Pol & $29.70-31.30$ & 150 & 1380 & 6.5 & 258 \\
\hline TSX-7 & 11.05 .2010 & Bermuda & N31.37-W69.54 & Quad-Pol & $29.70-31.30$ & 150 & 1380 & 6.9 & 62 \\
\hline TSX-8 & 05.03 .2013 & Mauritania & N18.78-W17.43 & Dual-Pol & $30.81-32.33$ & 150 & 1380 & - & - \\
\hline TSX-9 & 05.03 .2013 & Senegal & N13.91-W18.48 & Dual-Pol & $31.91-33.42$ & 150 & 1380 & - & - \\
\hline TSX-10 & 12.04 .2010 & Barcelona & N41.23-E2.11 & Quad-Pol & $33.04-34.50$ & 150 & 1380 & - & - \\
\hline TSX-11 & 28.04 .2010 & Southeast Yakutat & N58.20-W137.96 & Quad-Pol & $36.18-37.54$ & 150 & 1380 & 7.2 & 164 \\
\hline TSX-12 & 09.05 .2010 & Southeast Yakutat & N58.20-W137.96 & Quad-Pol & $36.18-37.54$ & 150 & 1380 & 5.7 & 350 \\
\hline TSX-13 & 23.04 .2010 & Pratas Island & N20.67-E116.92 & Quad-Pol & $39.03-40.29$ & 150 & 1380 & - & - \\
\hline TSX-14 & 25.04 .2010 & South Hecate Strait & N52.37-W129.81 & Quad-Pol & $40.02-41.25$ & 150 & 1380 & 13.1 & 140 \\
\hline TSX-15 & 06.05 .2010 & South Hecate Strait & N52.37-W129.81 & Quad-Pol & $40.02-41.25$ & 150 & 1380 & 6.7 & 318 \\
\hline TSX-16 & 17.03 .2013 & Gulf of Guinea & N0.24-E7.97 & Dual-Pol & $43.45-44.58$ & 150 & 1380 & - & - \\
\hline TSX-17 & 21.04 .2010 & North Sea & N54.38-E7.05 & Quad-Pol & $44.37-45.48$ & 150 & 1380 & - & - \\
\hline
\end{tabular}

\section{DATA SET Description}

87 ceiver could be an important limiting factor for proper sea clut88 ter characterization (statistical and polarimetric backscattering 89 fitting), particularly in the case of quad-polarized acquisitions 90 and for the spaceborne high-incidence region $\left(36^{\circ}-45^{\circ}\right)$ due 91 to the reduced SNR conditions. The $\sigma^{0}$ model, i.e., XMOD2 92 [6], optimized from TSX/TDX dual-pol data, provides a close 93 fitting to the measured data also for the quad-pol case, where 94 remaining calibration issues on the experimental DRA mode 95 may induce additional errors [7].

96 The statistical analysis based on the computation of the 97 normalized intensity moments (NIM) [8], and complemented 98 by the log-cumulants' study [9], [10] confirms deviation from 99 Gaussian statistics for the high-resolution X-band system [8], 100 [10]-[13], and mainly at incidence angles below $36^{\circ}$, where 101 sufficient SNR is available.

102 To evaluate the scattering properties of the sea when imaged 103 by TSX, the polarimetric roll-invariant parameters, i.e., entropy $104 H$ and mean scattering angle $\bar{\alpha}$ described in [14], as well 105 as the polarization ratio (PR) metrics [10], [15], have been 106 exploited. The results indicate that most of the acquisitions lie 107 in the low to medium random surface scattering region, mainly 108 due to the important contribution of nonpolarized returns, as 109 indicated by $H / \bar{\alpha}$ and PR analyses. The proposed X2-Bragg 110 model shows a good fitting with the experimental data in the $111 H / \bar{\alpha}$ plane, providing a mechanism to model the nonpolarized 112 returns in terms of $\beta_{1}$ parameter, which is an indicator of the 113 surface roughness (breaking waves events are known to cause 114 an increase in surface roughness [8], [16]).

115 The remainder of this paper is organized as follows. In 116 Section II, the TSX data set is presented. The methodology fol117 lowed to perform the sea clutter characterization is introduced 118 in Section III. In Section IV, the radiometric analysis in terms 119 of radar backscattering coefficient is presented. Later on, the 120 statistical description of the experimental data set is analyzed 121 in Section V. To end up the transversal characterization, a com122 plete polarimetric analysis is carried out in Section VI. Conclu123 sions are drawn in Section VII. A comprehensive derivation of 124 the proposed X2-Bragg model and its theoretical evaluation are 125 considered in Appendixes A and B.
The main parameters of the stripmap products used in the 127 analysis are summarized in Table I. The different data sets are 128 made up of acquisitions from both the TSX and TDX instru- 129 ments. Different aspects were considered for the selection of 130 the data under analysis: 1) covering the spaceborne SAR oper- 131 ational range of incidence angles from $20^{\circ}$ to $45^{\circ} ; 2$ ) avoiding 132 heterogeneity in the reflectivity over the imaged area; 3 ) avoid- 133 ing regions with high density of maritime traffic and/or marine 134 littorals (because of the high ambiguities' impact in the DRA 135 acquisition); and 4) when possible, polarimetric acquisition is 136 preferred over single polarization.

The available fully polarimetric TSX data were acquired 138 during the experimental DRA mode campaign, April and May 139 2010. It is well known that sea or ocean conditions, and hence 140 their radar response, could be quite different depending on the 141 geographical location of the marine scenario under analysis; 142 this is why it has been tried to sample the acquisitions over dif- 143 ferent oceans (Atlantic and Pacific) and seas (North, Mediter- 144 ranean, and China). For the nominal dual-polarization stripmap 145 products, the absolute and relative radiometric accuracies are 146 0.6 and $0.3 \mathrm{~dB}$, respectively; whereas for the quad-polarization 147 products, there is no specification, since it is an experimental 148 mode with few dedicated campaigns and additional investiga- 149 tions on the DRA calibration issues need to be considered [7]. 150

Portions of the images of three representative data takes 151 (near/TSX-4, middle/TSX-7, and far range/TSX-17) are shown 152 in Fig. 1; only copolarized channels $\left(S_{\mathrm{HH}}\right.$ and $\left.S_{\mathrm{VV}}\right)$ are consid- 153 ered since the cross-polar returns are corrupted by the thermal 154 noise. Data takes TSX-4, TSX-7, and TSX-17 correspond, 155 respectively, to high-, medium-, and low-SNR scenarios, with 156 mean values of $16.3,10.9$, and $3.7 \mathrm{~dB}$ for the copolar $S_{\mathrm{HH}} 157$ channel. The average SNR estimated values are summarized in 158 Table II for the different data takes, where the cross-polarized 159 returns have low SNR (most of them around 1-5 dB). Data 160 takes TSX-11 and beyond (incidence angle above $36^{\circ}$ ) have a 161 higher impact of the thermal noise, as indicated by the reduced 162 SNR conditions, even for the copolar channels. For TSX-4 163 [see Fig. 1(a) and (b)], the local surface wind produces an 164 
(a)

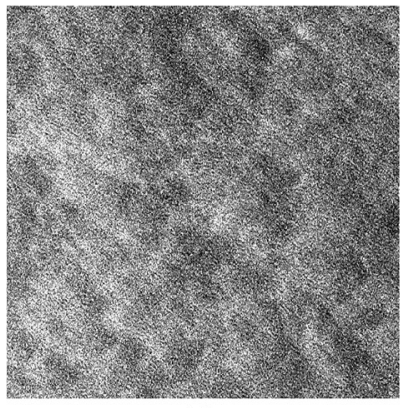

(c)

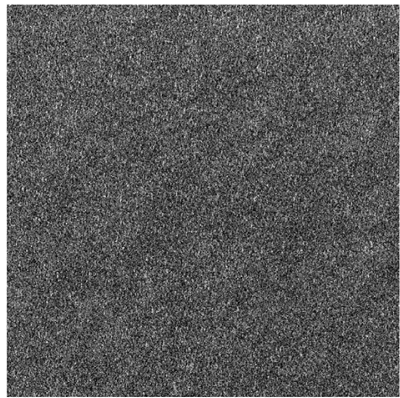

(e)

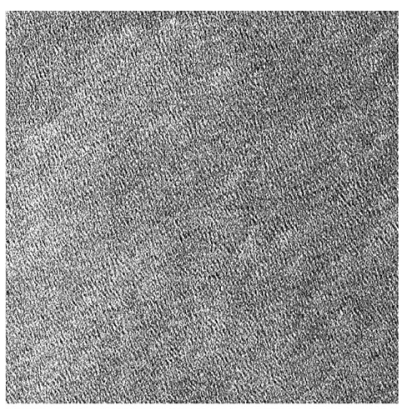

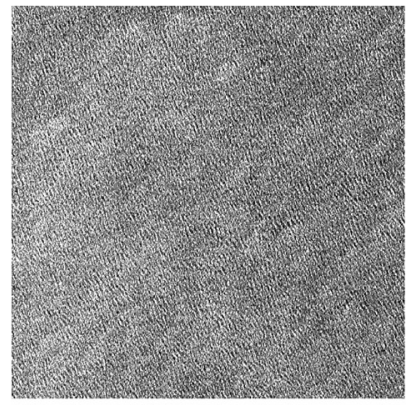

(b)

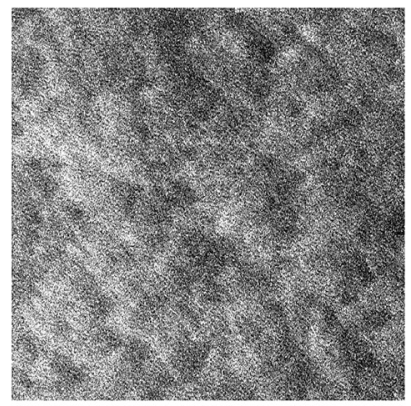

(d)

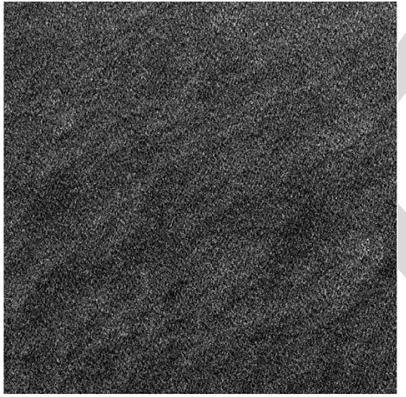

(f)
Fig. 1. Multilook $(11 \times 11)$ patches $(512 \times 512$ pixels $)$. (a) and (b) $S_{\mathrm{HH}}$ and $S_{\mathrm{VV}}$ for TSX-4 $\left(v_{\mathrm{win}}=6.3 \mathrm{~m} / \mathrm{s}\right.$ and $\left.\theta_{\mathrm{win}}=331^{\circ}\right)$. (c) and (d) $S_{\mathrm{HH}}$ and $S_{\mathrm{VV}}$ for TSX-7 $\left(v_{\text {win }}=6.9 \mathrm{~m} / \mathrm{s}\right.$ and $\left.\theta_{\text {win }}=62^{\circ}\right)$. (e) and (f) $S_{\mathrm{HH}}$ and $S_{\mathrm{VV}}$ for TSX-17 (no wind information).

TABLE II

Estimated SNR Average VALUeS For the TSX Data TAKES

\begin{tabular}{|c|c|c|c|c|c|}
\hline \multirow{2}{*}{ ID } & \multirow{2}{*}{$\boldsymbol{\gamma}[\mathbf{d e g}]$} & \multicolumn{4}{|c|}{ SNR [dB] } \\
\cline { 3 - 6 } & & $\boldsymbol{S}_{\boldsymbol{H} \boldsymbol{H}}$ & $\boldsymbol{S}_{\boldsymbol{V} \boldsymbol{V}}$ & $\boldsymbol{S}_{\boldsymbol{H} \boldsymbol{V}}$ & $\boldsymbol{S}_{\boldsymbol{V} \boldsymbol{H}}$ \\
\hline TSX-1 & $19.85-21.74$ & 23.0 & - & 4.8 & - \\
\hline TSX-2 & $22.43-24.26$ & 19.6 & - & 4.3 & - \\
\hline TSX-3 & $23.75-25.49$ & 17.7 & 17.9 & 6.8 & 6.9 \\
\hline TSX-4 & $23.75-25.49$ & 16.3 & 16.5 & 5.9 & 6.1 \\
\hline TSX-5 & $24.80-27.99$ & 11.7 & - & - & - \\
\hline TSX-6 & $29.70-31.30$ & 8.7 & 9.9 & 3.8 & 4.0 \\
\hline TSX-7 & $29.70-31.30$ & 10.9 & 11.6 & 4.2 & 4.3 \\
\hline TSX-8 & $30.81-32.33$ & 9.2 & - & 4.0 & - \\
\hline TSX-9 & $31.91-33.42$ & 9.1 & - & 4.1 & - \\
\hline TSX-10 & $33.04-34.50$ & 6.5 & 8.6 & 0.9 & 1.3 \\
\hline TSX-11 & $36.18-37.54$ & 5.1 & 6.6 & 2.6 & 2.6 \\
\hline TSX-12 & $36.18-37.54$ & 5.3 & 6.2 & 3.2 & 3.3 \\
\hline TSX-13 & $39.03-40.29$ & 6.4 & 8.9 & 2.8 & 3.4 \\
\hline TSX-14 & $40.02-41.25$ & 5.8 & 8.8 & 2.6 & 3.4 \\
\hline TSX-15 & $40.02-41.25$ & 3.5 & 4.5 & 2.5 & 2.4 \\
\hline TSX-16 & $43.45-44.58$ & 2.8 & - & 2.2 & - \\
\hline TSX-17 & $44.37-45.48$ & 3.7 & 6.2 & 2.3 & 2.4 \\
\hline
\end{tabular}

165 appreciable wave pattern. For TSX-7 [see Fig. 1(c) and (d)], a 166 more turbulent scenario is observed with a front propagating 167 mainly in the diagonal direction. Turbulence, such as eddies or upwellings, could produce such response, bringing also 168 some organic material (surfactants) to the surface [17]. At the 169 highest incidence angle region, i.e., TSX-17, the HH channel 170 has a noise-like pattern [see Fig. 1(e)] compared with VV [see 171 Fig. 1(f)], where a wave pattern is roughly appreciated.

Ground truth data from the historical database of the National 173 Data Buoy Center of the National Oceanic and Atmospheric 174 Administration and the Canadian Moored Bouy of the DFO are 175 available only for some acquisitions, as indicated in Table I. 176 The geographical location of the employed buoys matches the 177 center of the acquired images, but the temporal sampling rate 178 is not sufficient to determine the exact sea conditions. Typical 179 differences on time are between 30 and 60 min before or after 180 the acquisition time; however, in most cases, sea conditions are 181 quite stable in a 4-h window around the acquisition time.

182

\section{Sea Clutter Characterization Methodology 183}

A software module has been implemented to automatically 184 perform a complete and exhaustive characterization of the 185 data based on the three-level approach (radar backscattering 186 coefficient $^{2}{ }^{2}$ statistics, and polarimetry). This analysis module 187 can accept as input a set of different data takes from different 188 sensors, where each is processed sequentially.

189

The first submodule performs a radiometric calibration, 190 based on the input product information as described in [18], 191 such that the intensity of the pixels at its output is related to $\sigma^{0} 192$ values. ${ }^{3}$ In the next block, the area of interest (AOI) is selected, 193 which is, to date, carried out simply by a visual inspection of 194 the image product considering a rectangular AOI. Afterward, a 195 subregion characterization based on a sliding window over this 196 very AOI is performed. It is possible to define the size of such a 197 sliding window and the overlapping in both range and azimuth 198 dimensions.

For the statistical characterization and the normalized reflec- 200 tivity $\sigma^{0}$ estimation, a window considering the whole azimuth 201 strip for each range bin (range line basis) within the AOI has 202 been selected. Therefore, the dependence of different descrip- 203 tors with the incidence angle can be studied. For polarimet- 204 ric decomposition, a rectangular sliding window of $11 \times 11205$ without overlapping has been used to avoid mixing different 206 scattering mechanisms and obtain adequate speckle filtering 207 [19]. As proposed in [5], an additive noise filtering (NF) (for 208 quad-pol data) can be performed in order to reduce the noise 209 impact. Then, the polarimetric description is performed before 210 and after NF.

Prior to sub-AOI dependent characterization, specific filter- 212 ing on the data can be optionally completed, ${ }^{4}$ such that the 213 impact of man-made structures present in the open sea (such as 214 vessels, oil platforms, and wind farms) is minimized, avoiding 215

\footnotetext{
${ }^{2}$ In the literature, the radar backscattering coefficient is also referred as normalized radar cross section (NRCS).

${ }^{3}$ It must be noted that those pixels with negative intensities, once the calibration is performed, are excluded from the subsequent characterization.

${ }^{4}$ This filtering method has been considered only for those data takes where a high density of high-reflectivity man-made targets is expected, based on a visual prescreening approach, taking into account the a priori geographical location of the region being analyzed; otherwise, and depending on the filtering threshold, the sea clutter data characterization could be impaired.
} 
216 corruption of the data analysis. A simple filter approach has 217 been implemented: those pixels, whose magnitude $y_{i}$

$$
y_{i} \geq \mu_{\text {subAOI }}+\beta \sigma_{\text {subAOI }}
$$

218 exceeds the mean statistical value $\mu_{\text {subAOI }}$ in a given number 219 of times $\beta$ the standard deviation $\sigma_{\text {subAOI }}$, are discarded. Both 220 statistical moments are estimated from the available samples 221 within the sub-AOI, whereas the $\beta$ term is experimentally 222 adjusted, with typical values between 2 and 6 .

\section{IV. RADIOMETRIC ANALYSIS (NRCS)}

224 Most of the available semiempirical models of $\sigma^{0}$ (NRCS) 225 are optimized for the low grazing region, based on ground226 based radar observations. Therefore, they are not applicable for 227 spaceborne SAR acquisitions typically at $20^{\circ}-45^{\circ}$ of incidence 228 angle. Recently, two models have been proposed: the first 229 one, the Naval Research Laboratory (NRL) [20], is based on 230 the Nathanson reference tables in [21], covering the region 231 of $30^{\circ}-90^{\circ}$; the second one, named XMOD2 [6], has been 232 optimized from TSX/TDX copolar data, for incidence angles 233 from $20^{\circ}$ to $45^{\circ}$. XMOD2 provides $\sigma^{0}$ values at $\mathrm{VV}$, and thus, 234 PR models should be used to retrieve the HH component. In [6], 235 three models (T-PR, E-PR, and X-PR) have been adjusted for 236 copolarized TSX data.

237 The estimated $\sigma^{0}$ for the different data takes is depicted as 238 a function of the incidence angle in Fig. 2 for the copolar 239 channels. A sliding window in a range line basis is used, i.e., $240 \sigma^{0}$ is averaged using all the azimuth samples in a range bin. The 241 sea return decreases as a function of incidence angle, where the $242 S_{\mathrm{VV}}$ channel has, in general, a higher backscattering than $S_{\mathrm{HH}}$. 243 Cross-polar channels, not reported due to space limitations, 244 have low $\sigma^{0}$ values, close to noise level. NESZ is also reported 245 for the different data takes as dotted black lines in Fig. 2, 246 which can help in understanding the impact of the thermal noise 247 on each data take and thus the related SNR metric (estimated 248 average values are reported in Table II). Comparing the NESZ 249 of quad-pol data with dual-pol acquisitions (e.g., TSX-6 versus 250 TSX-8), a degradation around $3 \mathrm{~dB}$ is observed due to the 251 DRA mode operation. Therefore, the increase on NESZ (or 252 decrease in SNR) for quad-pol data will impair the proper 253 characterization of the sea clutter using TSX data. For some 254 data takes (e.g., TSX-11, TSX-12, TSX-15, and TSX-16), and 255 due to their reduced SNR conditions $(\approx 2-5 \mathrm{~dB})$, the effect of 256 the antenna elevation pattern at the edges of the corresponding 257 subswath can be clearly appreciated.

258 In Fig. 2, the range of copolar values predicted by the 259 NRL and XMOD2 models for a sea surface wind speed range 260 of $0.1-20 \mathrm{~m} / \mathrm{s}$ is indicated by the shaded regions, delimited 261 by dash-dot-dot and dashed gray lines, respectively. In the 262 XMOD2 case, an average of the upwind, crosswind, and down263 wind conditions has been considered. Moreover, the expected 264 values for $\mathrm{HH}$ have been also averaged for the different PR 265 models (T-PR, E-PR, and X-PR). For data takes with ground 266 truth information, the NRL model has an average deviation 267 w.r.t. the estimated values of 6.92 and $5.11 \mathrm{~dB}$ for $S_{\mathrm{VV}}$ and $S_{\mathrm{HH}}$ 268 channels, respectively; whereas XMOD2 presents a mean error 269 of $0.98 \mathrm{~dB}$ for $S_{\mathrm{VV}}$; and for the $S_{\mathrm{HH}}$ channel, the average de-

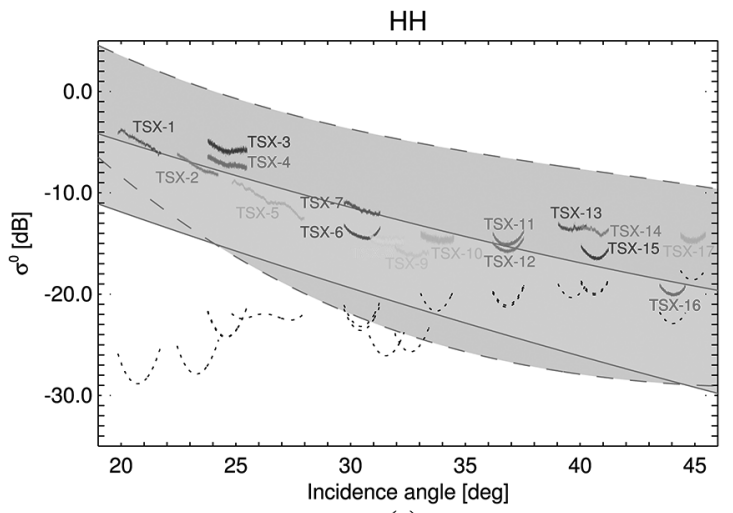

(a)

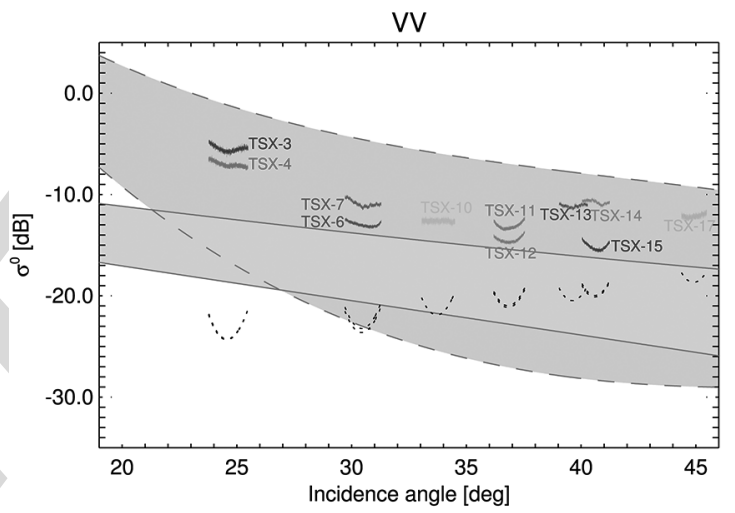

(b)

Fig. 2. Estimated $\sigma^{0}$ as a function of the incidence angle, using a sliding window in a range line basis with no overlap. (a) HH. (b) VV. The different data takes correspond to different colors; the (dotted black line) estimated NESZ is also plotted, and the shaded regions represent the predicted values from the NRL (delimited by dash-dot-dot lines) and XMOD2 (delimited by dashed lines) models, for a wind speed variation of $0.1-20.0 \mathrm{~m} / \mathrm{s}$ (average value between upwind, crosswind, and downwind is performed for XMOD2 and an average over the different PR models for $\mathrm{HH}$ extraction).

viations are 1.12, 1.23, and $2.63 \mathrm{~dB}$ for T-PR, E-PR, and X-PR, 270 respectively. This divergence between the VV and HH returns 271 (also among the different PR models) has been observed when 272 retrieving wind speeds from TSX/TDX VV-only data and HH- 273 only data as noted in [6], exploiting XMOD2 and PR models. 274

Since the available information of the sea conditions does not 275 correspond precisely to the time of acquisition, some error could 276 be introduced in the inversion of $\sigma^{0}$ for the theoretical models. 277 The NRL parametrization uses only two Nathanson tables [21], 278 at $30^{\circ}$ and $60^{\circ}$ of incidence angle, whereas five tables are used 279 to model reflectivity at incidence angles greater than $80^{\circ}$ [20]. 280 Therefore, a degradation in properly modeling $\sigma^{0}$ is expected 281 when considering typical spaceborne geometries with $20^{\circ}-45^{\circ} .282$

In case of XMOD2, which agrees much better with the 283 estimated values, the source of discrepancy should be also 284 related to the fact that this model has been optimized for a 285 given set of TSX/TDX copolarized acquisitions, not for quad- 286 pol data. Moreover, the quality of such experimental data (DRA 287 operation) should be carefully reviewed, particularly taking 288 into account that some calibration issues have been highlighted 289 when retrieving the fore and aft signals from the sum and 290 difference ones [4]. The calibration procedure proposed in [4] 291 has been applied for the along-track interferometric mode, 292 where the receive polarizations of the two halves of the antenna 293 
TABLE III

Statistical Distributions Used to Fit the Sea Clutter AMPLITUDE $Y$ (REFERENCES TO THE ESTIMATION METHODS ARE INDICATED)

\begin{tabular}{|c|c|c|c|}
\hline Distribution & Probability density function (PDF) & Parameters & Ref. \\
\hline \hline Log-normal & $p_{Y}(Y)=\frac{1}{Y \sqrt{2 \pi \sigma^{2}}} \exp \left(-\frac{(\ln Y-\mu)^{2}}{2 \sigma^{2}}\right)$ & $\begin{array}{l}\mu \text { (location) } \\
\sigma \text { (scale) }\end{array}$ & {$[12]$} \\
\hline Weibull & $p_{Y}(Y)=\beta \frac{Y^{\beta-1}}{a^{\beta}} \exp \left(-\left(\frac{Y}{a}\right)^{\beta}\right)$ & $\begin{array}{l}\beta \text { (shape) } \\
a \text { (scale) }\end{array}$ & {$[22]$} \\
\hline K-distribution & $p_{Y}(Y)=\frac{4 b \frac{(v+1)}{2} Y^{v}}{\Gamma(v)} K_{v-1}(2 Y \sqrt{b})$ & $\begin{array}{l}v \text { (shape) } \\
b \text { (scale) }\end{array}$ & {$[23]$} \\
{$[24]$}
\end{tabular}

${ }^{1} \Gamma(\cdot)$ is the gamma function and $K_{n}(\cdot)$ refers to the modified Bessel function of second kind and order $n$.

294 are the same, but no investigations have been made for the 295 quad-pol case (different polarizations in the fore and aft halves). 296 Hence, and as stated in the TSX product specification [7], 297 the DRA specific instrument characteristics have to be further 298 investigated for full calibration. From these results, it is clear 299 that an optimization of the XMOD2 and different PR models 300 is needed for quad-polarized data, using a wide data set with 301 in situ buoy measurements and considering further efforts in 302 instrument calibration for the fully polarimetric operation.

\section{3}

\section{Statistical Analysis}

304 For low-resolution radars and grazing angles typically above $30510^{\circ}$ (incidence angles less than 80 ), the amplitude (or magni306 tude) statistics of the sea returns have a "speckle"-like pattern 307 [8], [11], i.e., Rayleigh distributed in amplitude. For higher 308 resolution systems, the statistical description of the echo returns 309 deviates from the Gaussian hypothesis, having a target-like 310 response with more heavy-tailed distributions [8], [11]. This 311 non-Gaussian clutter has been commonly described, among 312 others, by log-normal and Weibull distributions [12], [13]. $313 \mathrm{~K}$-distribution, which provides also a good description of the sea 314 clutter magnitude for high-resolution radars [8], [11], has been 315 theoretically justified on the basis of the compound model [8].

316 Table III lists the probability density functions (PDFs) and 317 the related parameters for the log-normal, Weibull, and K distri318 butions. $Y$ refers to the amplitude (absolute value) of the single319 look $i$ th polarimetric channel $S_{i}$ (complex data), either $S_{\mathrm{HH}}$, $320 S_{\mathrm{VV}}$, or $S_{\mathrm{HV}}$ (polarization states in reception and transmission 321 are indicated by the first and second subindexes, respectively). 322 Fig. 3(a) and (b) show the fitting of the considered distribu323 tions (K, Rayleigh, log-normal, and Weibull) to the $S_{\mathrm{HH}}$ chan324 nel's magnitude for data takes TSX-4 and TSX-17, respectively, 325 which correspond accordingly to high-SNR (16.3 dB) and low326 SNR (3.7 dB) scenarios. Only the results for a specific single 327 sub-AOI are shown, i.e., in this case, the data distributions are 328 computed using all the azimuth samples for a specific single 329 range bin. The distributions are plotted on a semilogarithm 330 scale, i.e., the clutter is expressed in decibels, which, for 331 calibrated data, refers to $\sigma^{0}$ (theoretical distribution fitting and 332 parametric estimation done in the linear domain).

333 For TSX-4, in the small incidence angle region, K-distribution 334 (dotted blue line with circle markers) fits best the data dis335 tribution (solid red line), particularly in the distribution's tail. 336 This is crucial, for instance, in GMTI operation, since the tail's 337 shape determines the detection threshold for a given false alarm

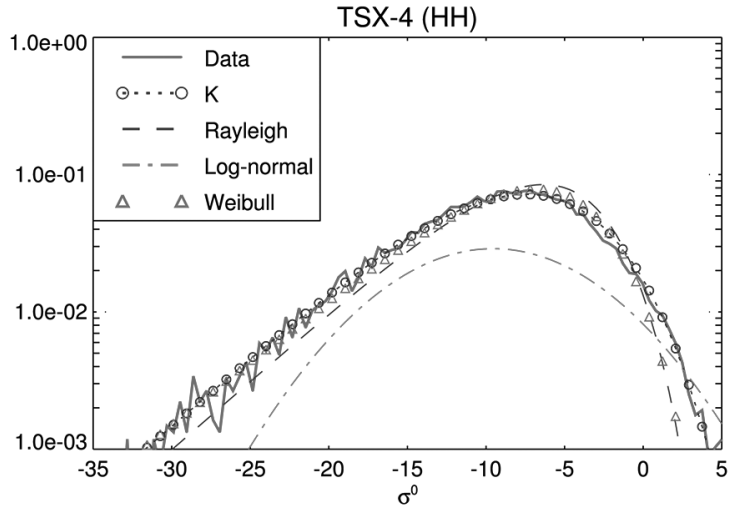

(a)

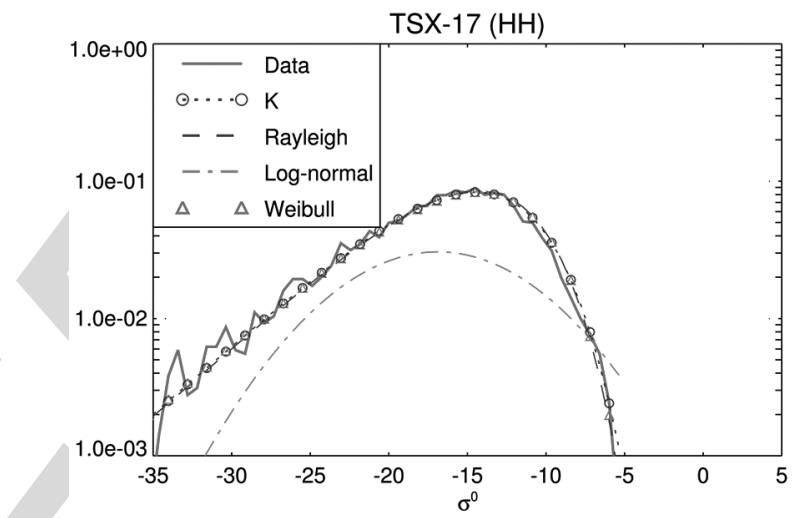

(b)

Fig. 3. Fitting of theoretical distributions (K, Rayleigh, log-normal, and Weibull) to the $S_{\mathrm{HH}}$ channel's magnitude (within a specific sub-AOI) for data takes (a) TSX-4 [SNR of $16.3 \mathrm{~dB}$ ] and (b) TSX-17 [SNR of $3.7 \mathrm{~dB}$ ].

rate. Weibull distribution shows good agreement with the data 338 only for smaller $\sigma^{0}$ values, whereas the log-normal does not fit 339 the data distribution. Similar results are obtained for the $S_{\mathrm{VV}} 340$ channel. In the cross-polar channel $S_{\mathrm{HV}}$, the three distributions 341 (K, Rayleigh, and Weibull) collapse to a complex Gaussian 342 distribution mainly due to the noise impact. For TSX-17, 343 the reduction in SNR, in comparison with TSX-4, leads to a 344 major contribution of the thermal noise, which is translated 345 into a more Gaussian-like data distribution. This behavior can 346 be recognized from the statistical fitting in Fig. 3(b), where 347 the $\mathrm{K}$ - and Weibull distributions tend to a Rayleigh one, i.e., 348 shape parameters $v>30$ and $\beta \approx 2$, accordingly. For the $S_{\mathrm{VV}} 349$ channel, there is an increase around $3 \mathrm{~dB}$ in SNR compared 350 with $\mathrm{HH}$ polarization, and K-distribution slightly departs from 351 Gaussianity, fitting best the data.

352

A good metric that allows deciding whether the data under 353 analysis are spikier or tend to a Gaussian distribution is the 354 shape parameter of the K-distribution, i.e., $v$. In Fig. 4, this 355 parameter is shown as a function of incidence angle for the 356 different data takes and copolar channels. ${ }^{5}$ Blacknell's method 357 has been used to estimate the shape and scale parameters of 358 the K-distribution. Similar results have been obtained using the 359 fractional moments method [23].

360

In the $S_{\mathrm{HH}}$ channel [see Fig. 4(a)], and for incidence angles 361 between $20^{\circ}$ and $33^{\circ}$, the shape parameter $v$ has a range of 362

${ }^{5}$ For those data takes at incidence angle above $35^{\circ}$ and with reduced SNR, only TSX-13 and TSX-14 are shown in Fig. 4 for clarity in the representation. 


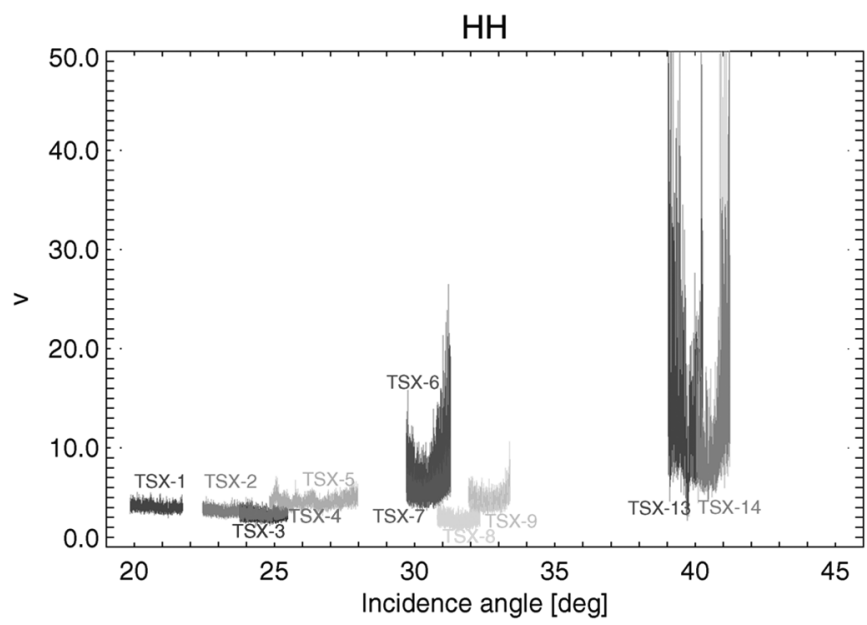

(a)

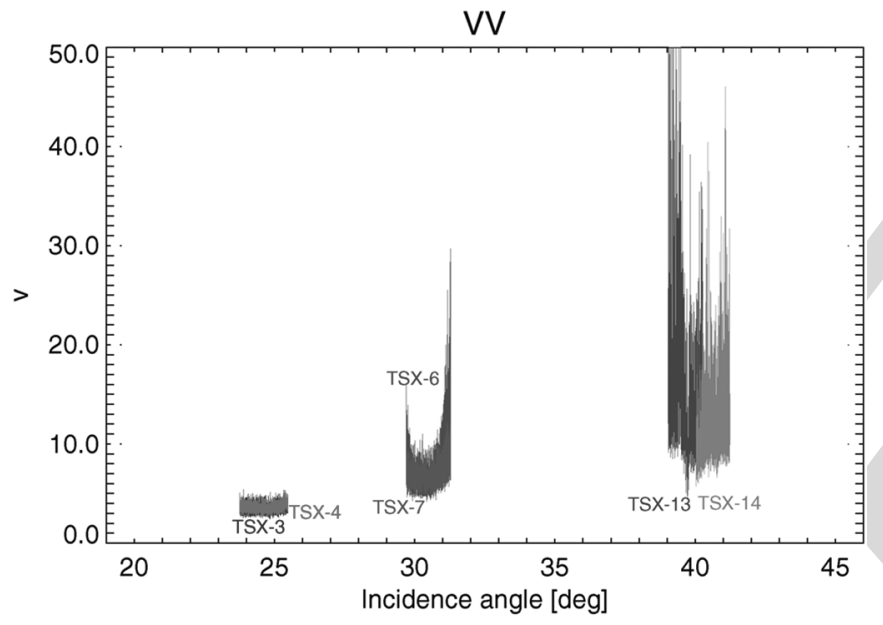

(b)

Fig. 4. Estimated shape parameter for a K-distributed amplitude fitting of the data as a function of the incidence angle, using a sliding window in a range line basis with no overlap. (a) HH. (b) VV. The different data takes are color-coded as in Fig. 2 (shape parameter estimated using Blacknell's approach).

363 3-10, with contained variation for each data take. For incidence 364 angles greater than $36^{\circ}$, the values of the shape parameter are 365 above 10, with much higher dispersion, related to the reduction 366 in SNR as incidence angle increases [see Fig. 2(a) and Table II]. 367 This is quite evident for TSX-16 and TSX-17, where noise 368 impact produces a variation on the shape parameter from 10 to 369 1000. For VV polarization [see Fig. 4(b)], similar trends are ob370 served, but in this case, the higher sea clutter returns in the high 371 incidence region reduce the shape parameter dispersion on data 372 take TSX-17, with values between 5 and 20. Comparing the 373 shape parameters of the polarimetric channels, different values 374 are obtained showing that the scalar product model (on which $375 \mathrm{~K}$-distribution is based) can be extended to a multivariative 376 product model, associating an individual texture variable per 377 polarimetric channel. For the cross-polar channels, high values 378 of the shape parameter are obtained with a high dispersion due 379 to the low sea backscattering contribution (close to NESZ).

380 One way to quantitatively evaluate the non-Gaussian behav381 ior of the data is by computing the NIM of the $n$th order [8], i.e.,

$$
\mathrm{NIM}_{n}=\frac{E\left\{Z^{n}\right\}}{E^{n}\{Z\}}
$$

where $Z$ refers to data intensity (or power), i.e., $Z=Y^{2}$, being 382 $Y=\left|S_{i}\right|$ the magnitude, and $E\{\cdot\}$ is the expectation operator. 383 Values of the $n$ th-order NIM above $n$ ! (Gaussian limit) are 384 indicators of spiky data.

In general, and for the copolar channels, the estimated 386 NIM (as a function of the estimated K-distribution shape pa- 387 rameter $v$ ) follows quite good the theoretical K-distribution 388 NIM trend, for the third and fourth orders, when considering 389 small to medium values of $v(<10)$, i.e., spikier clutter re- 390 turns. Therefore, the compound model theory that supports the 391 $\mathrm{K}$-distribution is able to describe the mechanism, which results 392 from a combination of Bragg scattering (from resonant capil- 393 lary waves) and whitecap scattering originated from breaking 394 waves [8]. The presence of the latter phenomena is known to 395 increase the spikiness of the clutter returns [8], associated to 396 smaller values of $v$ as observed for data takes with incidence an- 397 gle below $30^{\circ}$, with medium to high SNR conditions ( $>10 \mathrm{~dB}$ ), 398 as indicated in Table II.

Analogously, the estimated third- and fourth-order NIM 400 have been compared with the theoretical ones for Weibull and 401 log-normal distributions. Weibull presents, generally, a worse 402 matching with the theoretical trends for the copolar channels 403 compared with the K-distribution. When considering a log- 404 normal distribution fitting, a very poor matching between the 405 estimated NIM and the theoretical ones is obtained. In sum- 406 mary, K-distribution provides good fitting for specific TSX 407 acquisitions over the sea, particularly for low incidence angles, 408 where SNR conditions are favorable $(>10 \mathrm{~dB})$. In this region, 409 the lowest values of the K-distribution shape parameter are 410 obtained, indicating a much spikier behavior of the data, which 411 can be also related to the higher contribution of breaking wave 412 events, as analyzed in Section VI.

$\mathrm{K}$-distribution is among one of the statistical distributions 414 used to model and describe non-Gaussian statistics, based on 415 the well-known compound model. Under this hypothesis, the 416 complex signal can be characterized as a product between 417 the square root of a random variable $T$ (representing the tex- 418 ture) and an independent zero-mean complex Gaussian random 419 process ("speckle"). In this sense, the computation of the so- 420 called log-cumulants is very useful to evaluate the presence of 421 texture, i.e., non-Gaussian statistics [9]. Such analysis is typ- 422 ically performed through the log-cumulant diagram [9], [10], 423 where the second $\kappa_{2}$ (variance) and third $\kappa_{3}$ (skewness) log- 424 cumulants are plotted against each other. These log-moments 425 are computed as [10]

$$
\begin{aligned}
& \kappa_{2}=m_{2}-m_{1}^{2} \\
& \kappa_{3}=m_{3}-3 m_{1} m_{2}+2 m_{1}^{3}
\end{aligned}
$$

where $m_{n}$ is the $\log$-moment of order $n$, i.e., $m_{n}=427$ $1 / L \sum_{l=1}^{L}\left(\log Z_{l}\right)^{n}$, where $L$ intensity samples have been used. 428

Fig. 5 shows the log-cumulant diagram for the three rep- 429 resentatives acquisitions considered in Fig. 1 (from near to 430 far incidence angle regions). These plans are covered by five 431 (texture) distribution families, namely, Gamma (dash-dotted 432 line modeling a K-distribution in amplitude), inverse Gamma 433 (dashed black line), Beta (light gray shaded region), inverse 434 


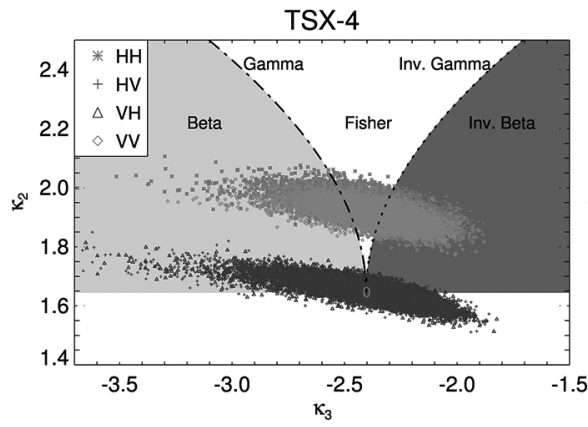

(a)

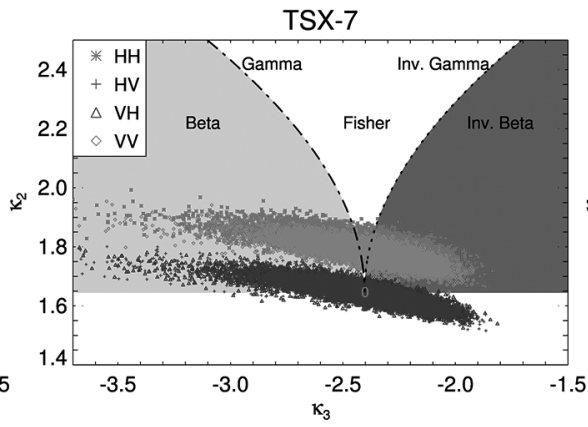

(b)

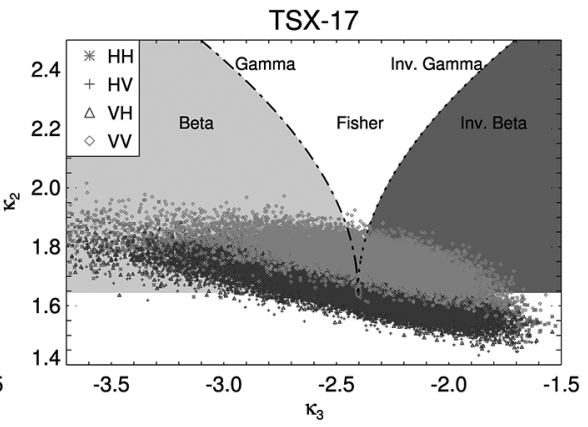

(c)

Fig. 5. Log-cumulant diagrams for different data takes: (a) TSX-4, (b) TSX-7, and (c) TSX-17. A sliding window in a range line basis with no overlap has been used (HH as red "x," HV as green "+," VH as " $\triangle$," and VV as gold "ऽ").

435 Beta (gray shaded region on the right), and Fisher (region in 436 between). The intersection point between these distributions 437 corresponds to no texture at all, i.e., Gaussian statistics.

438 The results in Fig. 5 are in line with the previous statistical 439 analysis: for TSX-4, in the near incidence angle region, the 440 copolar channels lie higher up in the diagram compared with 441 the cross-polar channels, indicating a deviation from Gaussian 442 statistics (spikier response). As the incidence angle increases, 443 the copolar channels move to lower regions in the diagram 444 (less texturized). For TSX-17, with the highest incidence angle, $445 \mathrm{VV}$ has more texture compared with $\mathrm{HH}$, as expected from the 446 K-distribution shape parameter study.

\section{7 448 \\ VI. ANALYSIS OF THE POLARIMETRIC AND SCATTERING FEATURES}

\section{A. SAR Polarimetry}

450 A polarimetric SAR (PolSAR) system measures the scatter451 ing matrix, which, for the monostatic case and on the Pauli 452 basis, can be expressed in vectorial notation as

$$
\mathbf{k}=\frac{1}{\sqrt{2}}\left[\begin{array}{lll}
S_{\mathrm{HH}}+S_{\mathrm{VV}} & S_{\mathrm{HH}}-S_{\mathrm{VV}} & 2 S_{\mathrm{HV}}
\end{array}\right]^{T}
$$

453 where $(\cdot)^{T}$ refers to the transpose operator, and $S_{i}$ are the com454 plex data of the $i$ th polarimetric channel. The distribution of $\mathbf{k}$ is 455 completely described by the Hermitian positive definite coher456 ency matrix $\mathbf{T}=E\left\{\mathbf{k k}^{H}\right\}$ under the Gaussian assumption [25], 457 where $(\cdot)^{H}$ is the transpose and complex conjugate operator.

458 The coherency matrix $\mathbf{T}$ is estimated from the data, such that 459 the speckle noise is filtered. The maximum-likelihood estimator 460 can be obtained as a spatial averaging (boxcar or multilook filter)

$$
\widehat{\mathbf{T}}=\frac{1}{L} \sum_{i=1}^{L} \mathbf{k}_{i} \mathbf{k}_{i}^{H}
$$

461 where $L$ refers to the number of looks or samples employed in 462 the estimation of $\mathbf{T}$, and $\mathbf{k}_{i}$ is the vector in the Pauli basis for 463 the $i$ th sample.

\section{B. Features Definition}

465 The main objective of the polarimetric study is to analyze the 466 scattering properties of the sea surface observed by the high467 resolution X-band TSX sensor. This way, two different sets of polarimetric features are considered. First, the eigendecompo- 468 sition of the coherency matrix allows extracting roll-invariant 469 parameters, such as the entropy $H$, anisotropy $A$, and mean al- 470 pha angle $\bar{\alpha}$, as originally proposed by Cloude and Pottier [14]. 471 $H$ measures the randomness of the scattering mechanism, $\bar{\alpha} 472$ provides physical information about the type of average scatter, 473 and $A$ (complementary to $H$ ) measures the relative significance 474 of the second and third eigenvalues. The $H / A / \bar{\alpha}$ features allow 475 a physical interpretation of the PolSAR data, and thus, their 476 correct estimation provides a reliable prediction of physical 477 parameters [5], [14]. This eigendecomposition-based analysis 478 has been successfully applied over the oceans to determine 479 polarimetric scattering mechanisms and for discrimination/ 480 classification of different types of slicks [16], [26], [27]. 481

To complement the eigendecomposition-based analysis, ad- 482 ditional polarimetric features exploiting the intensity of the 483 copolarized channels have been used, based on the $\sigma^{0}$ model 484 proposed by Kudryavtsev et al. [15]. As analyzed in [15], 485 [28], and [29], the Bragg theory is not fully able to explain 486 and represent the backscattering from the ocean, due to the 487 existence of sea spikes (in high-resolution radar data), which 488 induce large deviations of observed PR compared with the 489 theoretical Bragg one. This suggested that some mechanism 490 supporting non-Bragg scattering and connected with breaking 491 waves also contributes to the NRCS from the sea. The proposed 492 NRCS model in [15] is a superposition (in linear units) of a 493 regular sea surface, polarized component related to the Bragg 494 scattering $\left(\sigma_{p p}^{0 B}\right)$, and surface areas with enhanced roughness 495 produced by breaking waves, nonpolarized component linked 496 to non-Bragg scattering $\left(\sigma_{p p}^{0 n B}\right)$, i.e., $\sigma_{p p}^{0}=\sigma_{p p}^{0 B}+\sigma^{0 n B}$. It is 497 assumed that the nonpolarized component is the same for both 498 copolar channels.

As anticipated by several studies [15], [28], [29], the contri- 500 bution of the nonpolarized radar returns to the total NRCS can 501 be considerable, mainly due to the presence of breaking waves. 502 As suggested in [15] and [29], the computation of the PR 503

$$
\mathrm{PR}=\frac{\sigma_{\mathrm{HH}}^{0}}{\sigma_{\mathrm{VV}}^{0}}=\frac{\sigma_{\mathrm{HH}}^{0 B}+\sigma^{0 n B}}{\sigma_{\mathrm{VV}}^{0 B}+\sigma^{0 n B}}
$$

is an indicator of the presence of significant wave breaking 504 $(\mathrm{PR} \approx 1)$, deviating from the $\mathrm{PR}$ for the Bragg component 505 $p_{B}=\sigma_{\mathrm{HH}}^{0 B} / \sigma_{\mathrm{VV}}^{0 B}$. In this paper, $p_{B}$ is computed as the ratio 506 
507 between the perpendicular $\left(B_{\perp}\right)$ and parallel $\left(B_{\|}\right)^{6}$ Bragg 508 scattering coefficients according to [5]. It must be noted that 509 the effect of slope of the long tilting waves on the NRCS is 510 higher at the $\mathrm{HH}$ polarization than in $\mathrm{VV}$, particularly at large 511 incidence angles [15], and hence, this could lead to an increase 512 in PR not directly related to the breaking waves. Nevertheless, 513 it is complicated to separate the effect of breaking waves from 514 tilting waves, since, in the formation process of the former 515 ones, there is a progressive increase of the slope (generation 516 of a sloping wave front) correlated with a temporal increase 517 of the surface roughness [16]. In this sense, further studies 518 are required to carefully analyze the impact of both tilting and 519 breaking waves, trying to separate both processes.

\section{C. X-Bragg and Extended X-Bragg (X2-Bragg) Models}

521 It has been generally assumed that the radar sea echo returns 522 are mostly dominated by Bragg surface scattering; however, 523 some depolarization has been observed, as noted in [16], [26], 524 and [30]. Such nonpolarized returns have been found to sig525 nificantly contribute to the sea backscattering due to breaking 526 waves (increasing surface roughness), as analyzed in [15], 527 [28], and [29], particularly when increasing the frequency of 528 operation (and thus the resolution) [15].

529 Under these considerations, the application of the small per530 turbation model (SPM) for the polarimetric scattering analysis 531 would be too simplistic, as its validity range is limited to small 532 surface roughness conditions [5]. Therefore, the evaluation of 533 the X-Bragg model, originally proposed by Hajnsek et al. [5], 534 seems to be more reasonable, since it is a two-component 535 model, including a Bragg scattering term and a roughness in536 duced rotation symmetric disturbance. This way, depolarization 537 (nonpolarized components) and cross-polarized backscattering 538 effects are included. As proposed in [5] and [16], this can 539 be obtained modeling the surface as a reflection symmetric 540 depolarizer, randomly rotating the Bragg coherency matrix $\mathbf{T}$ 541 about an angle $\beta$ w.r.t. the local surface normal and averaging it 542 over a statistical distribution $p_{\beta}(\beta)$. For a uniform distribution $543 p_{\beta}(\beta)$ with zero mean and width $\beta_{1}$, the coherency matrix can 544 be expressed by [5]

$$
\mathbf{T}=m_{s}^{2}\left[\begin{array}{ccc}
C_{1} & C_{2} \operatorname{sinc}\left(2 \beta_{1}\right) & 0 \\
C_{2}^{*} \operatorname{sinc}\left(2 \beta_{1}\right) & C_{3}\left(1+\operatorname{sinc}\left(4 \beta_{1}\right)\right) & 0 \\
0 & 0 & C_{3}\left(1-\operatorname{sinc}\left(4 \beta_{1}\right)\right)
\end{array}\right]
$$

545 where $m_{s}$ is the backscatter amplitude related to the surface 546 roughness; sinc function refers to $\operatorname{sinc}(x)=\sin (x) / x$; and the 547 coefficients $C_{1}, C_{2}$ and $C_{3}$ can be expressed in terms of $B_{\perp}$ and $548 B_{\|}$[5]. These coefficients depend on the incidence angle $\gamma$ and 549 the dielectric constant $\epsilon=\epsilon^{\prime}-\jmath \epsilon^{\prime \prime}$. The latter can be obtained 550 from the model developed by Klein and Swift [31].

551 The width of the distribution $\beta_{1}$ models the roughness dis552 turbance of the surface and controls the level of cross-polarized 553 power and the polarimetric $\left(S_{\mathrm{HH}}+S_{\mathrm{VV}}\right)\left(S_{\mathrm{HH}}-S_{\mathrm{VV}}\right)$ coher554 ence [5]: for $\beta_{1}=0^{\circ}$ (smooth surface), the cross-polarized

\footnotetext{
${ }^{6}$ Note that, here, perpendicular and parallel are referenced w.r.t. the incidence plane.
}

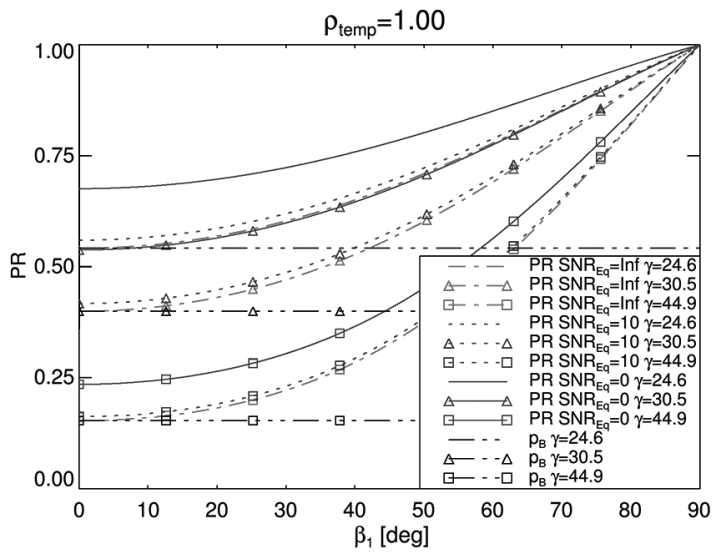

Fig. 6. $\mathrm{PR}$ of the $\mathrm{X}$-Bragg $\left(\mathrm{SNR}_{\mathrm{Eq}}=\infty\right)$ and $\mathrm{X} 2$-Bragg models is represented as a function of the roughness $\beta_{1}$ parameter for different incidence angles $\gamma$ and different noise conditions $\mathrm{SNR}_{\mathrm{Eq}}$ (an X-band system at $9.65 \mathrm{GHz}$, a sea temperature of $10^{\circ} \mathrm{C}$, and salinity of $35 \%$ are considered).

power is zero, and there is no depolarization effect (corre- 555 sponding to the "pure" Bragg or SPM model); with increasing 556 $\beta_{1}$ (increasing roughness), the $\mathrm{HV}$ term increases, and the 557 $\left(S_{\mathrm{HH}}+S_{\mathrm{VV}}\right)\left(S_{\mathrm{HH}}-S_{\mathrm{VV}}\right)$ coherence decreases until 0 in the 558 high roughness limit case of $\beta_{1}=90^{\circ}$ (azimuthally symmetric 559 surface). The breaking waves produce a temporal increase in 560 the surface roughness [8], [16], and this could be related to an 561 increase also in the $\beta_{1}$ term of the X-Bragg model. In fact, this 562 model has been used to derive a description of the polarimetric 563 behavior of a breaking wave, as presented in [16]. Therefore, 564 it is interesting to evaluate the applicability of the X-Bragg 565 model to SAR polarimetric sea data, which can be useful for 566 future studies trying to invert some geophysical parameters of 567 the ocean using polarimetric data.

The DRA acquisition mode [4], employed to obtain fully po- 569 larimetric images with an along-track interferometric configu- 570 ration, has a two-folded impact on measured data. On one hand, 571 as the receive antenna is halved, a direct consequence is a reduc- 572 tion on the received gain, which translates into higher values of 573 NESZ. On the other hand, the alternate transmission of horizon- 574 tal and vertical polarizations in a pulse-by-pulse basis jointly 575 with the along-track configuration (to receive simultaneously 576 $\mathrm{H}$ and $\mathrm{V}$ ) produces a temporal lag between different pairs of 577 channels. The different polarimetric channels should be spatial 578 aligned (coregistered), such that they observe the scene from 579 the same positions but at different times. During this time lag, 580 around $\tau_{\text {coreg. }}=1 \mathrm{~ms}$ for TSX $S_{\mathrm{HH}}$ and $S_{\mathrm{VV}}$ channels (or $S_{\mathrm{HV}} 581$ and $S_{\mathrm{VH}}$ ), the sea backscattered field decorrelates due to inter- 582 nal clutter motion. Typical decorrelation times of the sea are 583 between 8 and $10 \mathrm{~ms}$ for wind speeds of $15 \mathrm{~m} / \mathrm{s}$ down to $5 \mathrm{~m} / \mathrm{s}, 584$ [32]. For an X-band system at VV, the temporal coherence 585 $\rho_{\text {temp }}$ could be between 0.9 and 1.0 for a time lag around $1 \mathrm{~ms}, 586$ [32]. From the results presented in Section VI-D for TSX quad- 587 pol, it has been observed that both the noise level and the sea 588 clutter temporal decorrelation should be accounted for in the 589 X-Bragg model to properly characterize the sea clutter in po- 590 larimetric terms. Taking into account these issues, the X-Bragg 591 model has been extended to the X2-Bragg model (extended- 592 extended Bragg). The mathematical derivation of the X2-Bragg 593 


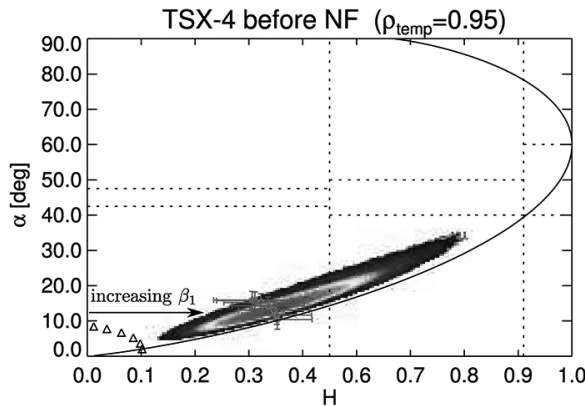

(a)

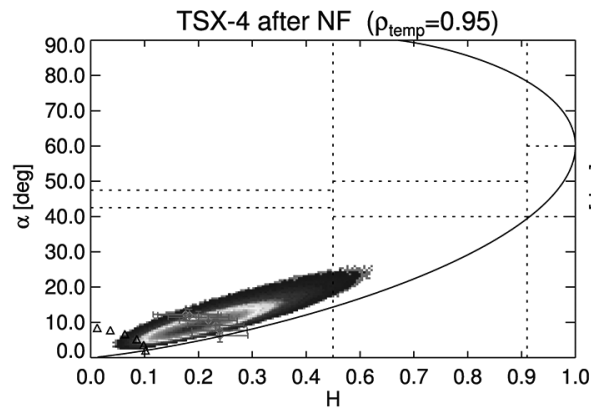

(d)

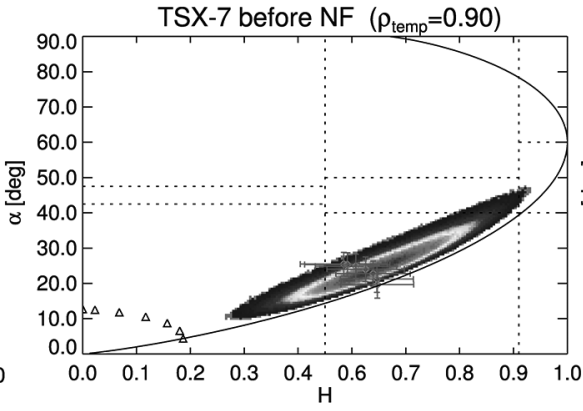

(b)

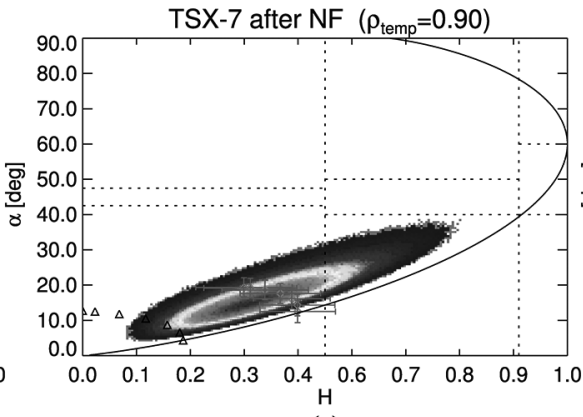

(e)

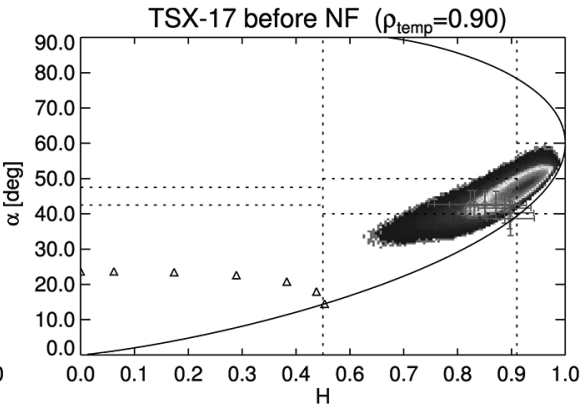

(c)

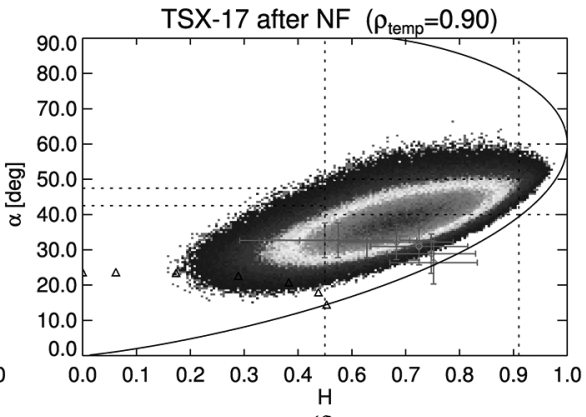

(f)

Fig. 7. Two-dimensional distributions on $H / \bar{\alpha}$ plane for different data takes (TSX-4, TSX-7, and TSX-17, from left to right), including the theoretical $\mathrm{X} / \mathrm{X} 2$-Bragg models $\left(\beta_{1}\right.$ variation from $0^{\circ}$ to $90^{\circ}$ and single dielectric constant for standard sea conditions of $10{ }^{\circ} \mathrm{C}$ and salinity of $35 \%$ ); black triangles

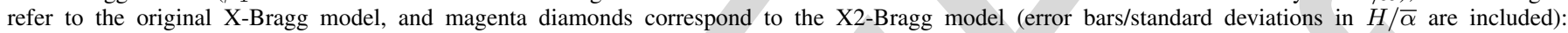
(a)-(c) before and (d)-(f) after NF. (a) and (b) TSX-4 with $\rho_{\text {temp }}=0.95$. (b) and (e) TSX-7 with $\rho_{\text {temp }}=0.90$. (c) and (f) TSX-17 with $\rho_{\text {temp }}=0.90$.

594 and a theoretical evaluation for different system/scenario con595 ditions are presented in Appendixes A and B, respectively.

596 In Fig. 6, the PR of the X-Bragg $\left(\mathrm{SNR}_{\mathrm{Eq}}=\infty\right)$ and the $597 \mathrm{X} 2$-Bragg is plotted as a function of $\beta_{1}$ for different incidence 598 angles $\gamma$ and effective ${ }^{7}$ signal-to-noise ratio $\mathrm{SNR}_{\mathrm{Eq}}$ conditions 599 (no temporal decorrelation has been considered). The horizon600 tal lines correspond to the PR for Bragg components $p_{B}$. It can 601 be seen how the PR approaches $p_{B}$ for $\beta_{1}$ close to zero (pure 602 Bragg) and increases to higher values as roughness condition 603 increases, with a value of 1 for complete depolarized scattering. 604 From [15] and [29], this trend can be related to higher contri605 bution of breaking wave events, which induce higher roughness 606 conditions. It must be also noted that, as the noise contribution 607 increases, there is also an increase in the PR, since the noise is 608 a common nonpolarized term affecting both copolar channels.

\section{D. Experimental Results}

610 An analysis of the scattering plots in the $H / \bar{\alpha}$ plane has been 611 carried out for the different data takes, without and with additive $612 \mathrm{NF}$ as proposed in [5]. Fig. 7 shows these 2-D distributions 613 for the three representative data takes (near to far incidence, 614 from left to right). Before NF, the dominant mechanism moves 615 from low entropy surface scattering ${ }^{8}$ at near incidence [see 616 Fig. 7(a)], passing by medium entropy surface scattering [see 617 Fig. 7(b)], ending up to high entropy region characterized by 618 random anisotropic scatters [see Fig. 7(c)], caused by the higher 619 noise contribution. Comparatively, NF produces a reduction

\footnotetext{
${ }^{7}$ Please refer to Appendix A for further details on the definition of this equivalent $\mathrm{SNR}_{\mathrm{Eq}}$.

${ }^{8}$ This region corresponds to zone 9 as designated by Cloude and Pottier [14], including surface scattering and specular scattering phenomena.
}

both in $H$ and $\bar{\alpha}$, leading to a dominant scattering in the low 620 entropy surface zone $\left(H<0.5\right.$ and $\left.\bar{\alpha}<40^{\circ}\right)$ for data takes 621 with incidence angle below $36^{\circ}$ (e.g., TSX-4 and TSX-7). 622

The distributions of the eigenvalues in Fig. 8 for the three 623 reference data takes are a good visual indicator of the polarimet- 624 ric mechanisms' evolution as a function of incidence angle. In 625 Fig. 8, the lowest eigenvalue $\left(\lambda_{4}\right)$ has been also included since 626 it provides information on the expected noise floor, such that the 627 dominant mechanism's relative contribution with respect to the 628 noise can be easily tracked. For TSX-4 [see Fig. 8(a)], there is a 629 clear dominant mechanism $\left(\lambda_{1}\right)$, surface scattering, as observed 630 from the $\alpha_{1}$ distribution in Fig. 9(a), centered around $5^{\circ}$. As 631 incidence angle increases, the first eigenvalue decreases. At 632 the farthest available incidence angle (TSX-17) [see Fig. 8(c)], 633 no clear dominant mechanism is present, being the distributions 634 of the eigenvalues closer to each other, such that a noise-like 635 mechanism is expected in accordance with the $\sigma^{0}$ and statistical 636 characterizations.

In general, the second mechanism almost keeps its relative 638 strength w.r.t. noise floor independently of incidence angle. In 639 Fig. 9, where the distributions of the individual $\alpha$ parameters 640 are shown, the related $\alpha_{2}$ with a similar trend as $\alpha_{3}$ lies between 641 $70^{\circ}$ and $90^{\circ}$. These values are representative of double-bounce 642 scattering, less probable in the sea backscattering. Considering 643 the X-Bragg model extension to account only for thermal 644 noise impact, parameters associated to the second mechanism 645 do not agree with the observations from experimental data: 646 on one hand, $\alpha_{2}$ corresponds to $90^{\circ}$ independently from $\beta_{1} 647$ and $\mathrm{SNR}_{\mathrm{Eq}}$; on the other hand, the expected level of $\lambda_{2}$ is 648 much closer to $\lambda_{3}$ than that observed from real data. These 649 considerations reinforce the need to include also the impact 650 of sea clutter temporal decorrelation between the polarimetric 651 
TSX-4

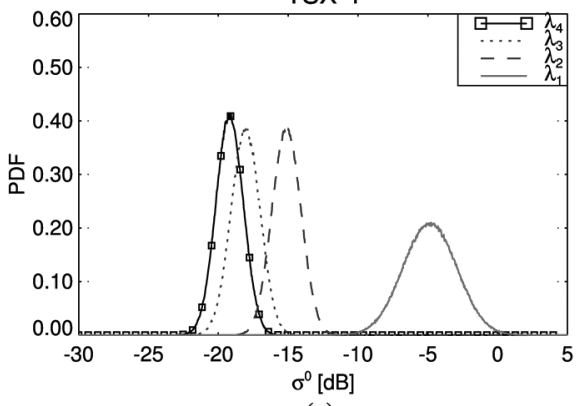

(a)
TSX-7

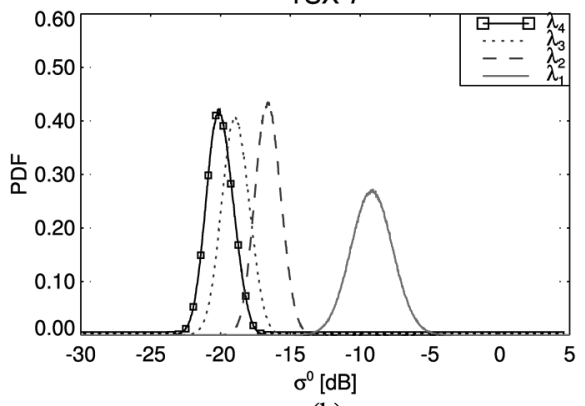

(b)
TSX-17

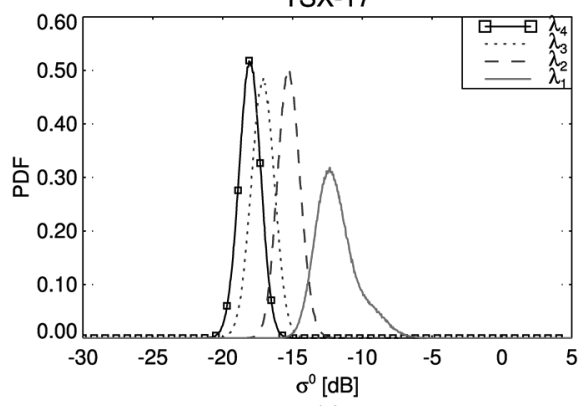

(c)

Fig. 8. Distribution of the eigenvalues, before NF, for different data takes. (a) TSX-4. (b) TSX-7. (c) TSX-17.

TSX-4

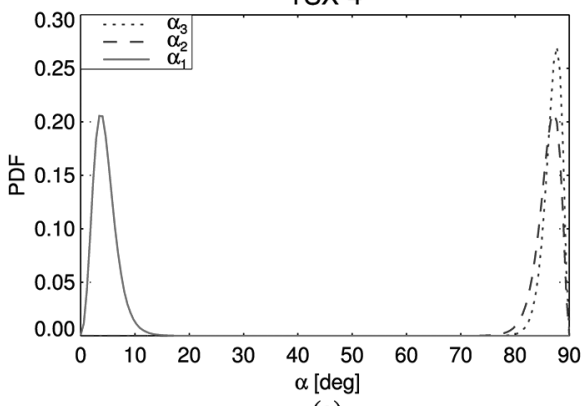

(a)

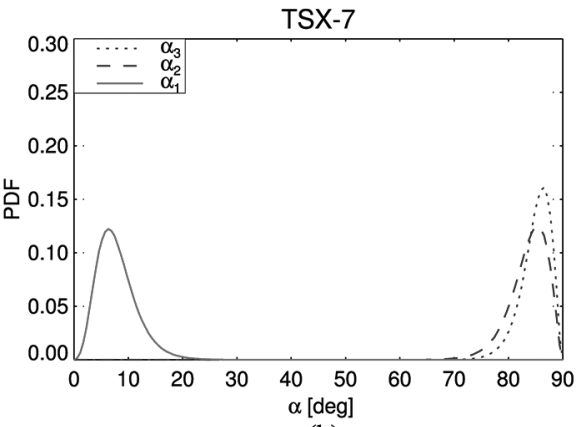

(b)

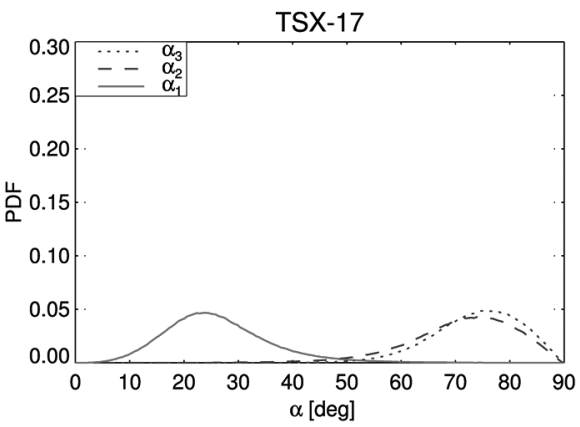

(c)

Fig. 9. Distribution of the alpha parameters, before NF, for different data takes. (a) TSX-4. (b) TSX-7. (c) TSX-17.

652 channels $\left(\rho_{\text {temp }}\right)$, intrinsically related to the DRA acquisition 653 mode itself, as proposed in the X2-Bragg model and described 654 in Appendixes A and B.

655 On top of the different $H / \bar{\alpha}$ distributions in Fig. 7, the 656 classical X-Bragg model, as black triangles, and the extended 657 model X2-Bragg, as magenta diamond symbols, are depicted 658 for different values of $\beta_{1}$ (from $0^{\circ}$ to $90^{\circ}$ in steps of $15^{\circ}$ ). 659 Standard deviations are also included for the X2-Bragg model 660 (related to $\mathrm{SNR}_{\mathrm{Eq}}$ estimation from the data). Standard sea 661 conditions have been assumed, i.e., $10^{\circ} \mathrm{C}$ and $35 \%$ salinity.

662 An important bias exists between the original X-Bragg model 663 and the data, after and, in particular, before NF. For X2-Bragg, 664 a much better matching between the theoretical model and the 665 experimental data is obtained. For TSX-4 [see Fig. 7(a)], a $666 \rho_{\text {temp }}$ of 0.95 provides a good matching between the theoretical 667 extended model and the data before NF, whereas for TSX-7 668 [see Fig. 7(b)], this value reduces to 0.90. In case of TSX-17 669 [see Fig. 7(c)], a higher bias is recognized, and reducing $670 \rho_{\text {temp }}$ below 0.9 does not provide any additional improvement: 671 from the theoretical analysis of the X2-Bragg model, higher 672 clutter decorrelation does not increase entropy for regions with $673 H>0.6$. Additive noise subtraction reduces entropy from 6740.92 to 0.7 , but the proposed extended model predicts an aver675 age alpha value below the one observed in the data. Therefore, 676 there might be an additional mechanism contributing to this 677 discrepancy, which is not considered in the X-Bragg model; 678 additional measurements with higher SNR conditions should 679 be analyzed to support this hypothesis.

680 The X2-Bragg model gets close to the centroid of the data 681 distributions in $H / \bar{\alpha}$ for values of $\beta_{1}$ different from zero, which means that no pure Bragg is being imaged, but there is a 682 depolarized term contributing also to the backscattering. An 683 increase in $\beta_{1}$ is related to higher surface roughness producing 684 depolarization and an increase in the entropy. To complement 685 the polarimetric study and gain additional insights into the 686 nonpolarized contribution of the sea backscattering, the PR for 687 the available quad-pol data takes is represented as a function of 688 the incidence angle in Fig. 10. The PR $p_{B}$ for the pure Bragg 689 model is also included as solid black line.

690

Before NF [see Fig. 10(a)], an important difference between 691 the estimated PR and $p_{B}$ exists, which means that an important 692 contribution on nonpolarized returns is presented, probably 693 related to breaking wave effects. Even for the high incidence 694 region (above $36^{\circ}$ ), where Bragg scattering is expected to be 695 dominant, as stated in [10], an appreciable divergence between 696 the PRs is observed (e.g., TSX-15 and TSX-17). Nevertheless, 697 as pointed out throughout this paper, the reduced SNR condi- 698 tions $(\approx 3-4 \mathrm{~dB})$ can explain this effect, since the thermal noise 699 is also a nonpolarized component common to both copolar 700 channels. This can be recognized from Fig. 10(b), where the 701 difference between PR and $p_{B}$ has been reduced for the high 702 incidence angle region after NF. ${ }^{9}$

703

Following the two-component NRCS model (Bragg/non- 704 Bragg) proposed by Kudryavtsev et al. [15], the relative 705

\footnotetext{
${ }^{9}$ In Fig. 10, the images have been radiometrically calibrated before the application of any further processing, i.e., the NESZ has been subtracted (in linear units) from the calibrated digital pixels, as proposed in [18]. From these results, it can be seen that there is still some residual noise that should be filtered out and that quad-pol data provide the means to do so.
} 


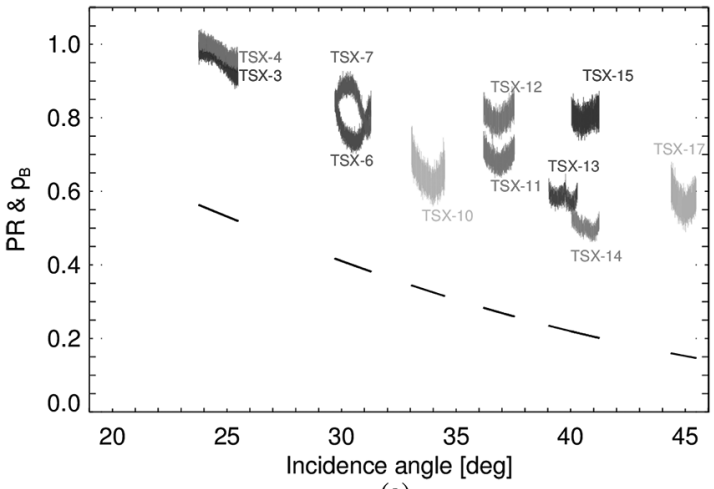

(a)

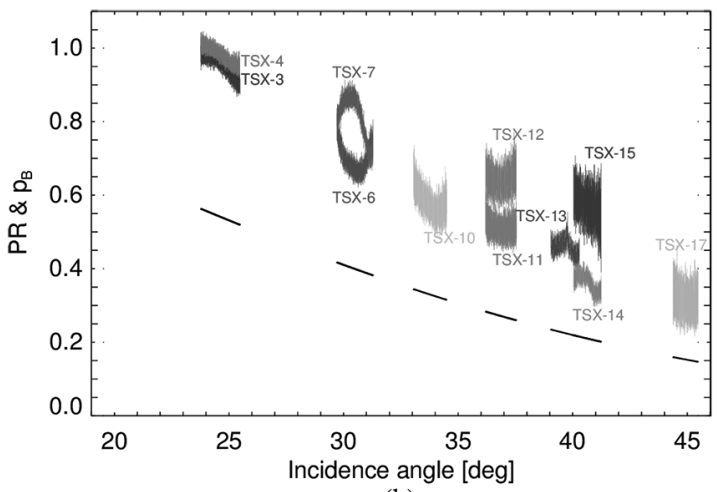

(b)

Fig. 10. PR as a function of the incidence angle for the different quad-pol data takes (color-coded as in Fig. 2): (a) before and (b) after NF; solid black lines refer to the PR for pure Bragg $p_{B}$.

706 contribution of the non-Bragg component with respect to the 707 total backscattered power for the $S_{\mathrm{HH}}$ channel $\left(\sigma^{0 n B} / \sigma_{\mathrm{HH}}^{0}\right)$ has 708 been computed, and it ranges from $50 \%$ to $90 \%$ (high to small 709 incidence angles), whereas for $\mathrm{VV}$, the figures $\left(\sigma^{0 n B} / \sigma_{\mathrm{VV}}^{0}\right)$ are 710 around $20 \%-70 \%$. These results are in line with the studies 711 carried out in [15] and [29]. For TSX-3 and TSX-4, $\sigma^{0 n B} / \sigma_{\mathrm{VV}}^{0}$ 712 and $\sigma^{0 n B} / \sigma_{\mathrm{HH}}^{0}$ have very similar trends close to the $90 \%$ of the 713 total backscattered power. In this very case, with the smallest 714 incidence angle $\left(25^{\circ}\right)$, specular returns may partially contribute 715 to the non-Bragg scattering [10], apart from possible breaking 716 wave effects.

717 A fitting of the X-Bragg and X2-Bragg models to the mea718 sured PR has been carried out. After NF, $\beta_{1}$ values providing 719 the minimum average error (between the models and the data) 720 within each quad-pol acquisition are summarized in Table IV. 721 As described in Section VI-B, an increase in $\beta_{1}$ is related 722 to a roughness enhancement and thus a higher depolarization 723 contribution. From Table IV, it can be generally stated that $\beta_{1}$ 724 reduces as a function of the incidence angle in accordance to 725 the decrease of the nonpolarized component. For the X2-Bragg 726 model, $\beta_{1}$ corresponds to the average value for three differ727 ent temporal decorrelation conditions assumed in the model 728 ( $\left.\rho_{\text {temp }}=1.0,0.95,0.90\right)$. Due to the presence of thermal noise 729 and possible temporal decorrelations, the X-Bragg model over730 estimates the surface roughness, when compared with the pro731 posed X2-Bragg model, particularly for the high incidence 732 region (above $36^{\circ}$ ).
TABLE IV

$\beta_{1}$ (Roughness) Providing the Minimum Average Error BetweEn Estimated PR From X/X2-BRAGg DATA FitTING AND MEASURED PR FOR QUAD-POL ACQUISITIONS AFTER NF

\begin{tabular}{|c|c|c|}
\hline ID & X-Bragg & X2-Bragg \\
\hline \hline TSX-3 & 84 & 83.3 \\
\hline TSX-4 & 87 & 87 \\
\hline TSX-6 & 59 & 56 \\
\hline TSX-7 & 73 & 72 \\
\hline TSX-10 & 51 & 46.7 \\
\hline TSX-11 & 51 & 46.7 \\
\hline TSX-12 & 63 & 62 \\
\hline TSX-13 & 50 & 44.7 \\
\hline TSX-14 & 41 & 32.7 \\
\hline TSX-15 & 62 & 60.7 \\
\hline TSX-17 & 43 & 34.3 \\
\hline
\end{tabular}

VII. CONCLUSION

A signal-based characterization of the sea/ocean returns im- 734 aged by TSX sensor has been carried out exploiting 17 data 735 sets, both dual- and quad-pol. The transversal study copes 736 with radiometric, statistical, and polarimetric analysis of the 737 experimental data. One of the goals of this paper is to provide 738 additional insights into proper modeling and characterization of 739 the sea clutter response when observed by TSX.

740

First, the different results indicate that, for the quad-polarized 741 data over maritime scenarios, the thermal noise impact can be 742 a limiting factor in the correct interpretation of the TSX data, 743 particularly for the low sea backscattering regions (incidence 744 angles above $36^{\circ}$ ). Second, taking into account the specific 745 DRA configuration to acquire quad-pol data, the induced tem- 746 poral decorrelation of the sea returns between the polarimetric 747 channels (due to the along-track configuration) is an additional 748 limiting factor that should be carefully considered. This effect, 749 combined with the additional thermal decorrelation, needs to be 750 properly accounted for when trying to fit polarimetric physical- 751 based models to the experimental data, in order to avoid mis- 752 interpretation. In this line, this paper proposes a new modeling 753 approach to include such system/scenario dependent limitations 754 applied specifically to the X-Bragg model and concreted in the 755 new X2-Bragg model.

756

The NRCS analysis shows that the semiempirical model 757 XMOD2 provides a fair fitting to the measured $\sigma^{0}$. Neverthe- 758 less, further studies are required to obtain a refined tunning of 759 the XMOD2 coefficients from quad-pol TSX data sets for a 760 wide range of sea conditions, ensuring that adequate calibration 761 of the DRA mode can be accomplished.

762

Deviation from the Gaussian statistics for the TSX sea data 763 is observed, when sufficient SNR is available and for small 764 to medium incidence angles (below 36 ${ }^{\circ}$ ). This means spikier 765 returns and thus heavy-tailed amplitude distributions. The log- 766 cumulant analysis shows that, for TSX, the ocean has high 767 texture due to the higher resolution of the sensor coupled with 768 the higher operating frequency (higher sensitivity to surface 769 roughness variation). These results are in line with the study 770 of the PR, which indicates an important contribution of non- 771 Bragg scattering (depolarization), particularly for small inci- 772 dence angles. As indicated in [15], [28], and [29], the presence 773 of breaking wave events, increasing surface roughness and 774 producing spikier returns, can explain this high depolarized 775 
776 contribution. These nonpolarized returns tend to reduce as the 777 incidence angle increases.

778 Exploiting the fully polarimetric information through the $779 H / \bar{\alpha}$ plane indicates that, for small incidence angles, the 780 dominant scattering mechanism corresponds to low to medium 781 random surface. The proposed X2-Bragg model proves a quite 782 good fitting to the data in the $H / \bar{\alpha}$ plane. Therefore, the type 783 of average mechanism and the relative strength between the 784 involved scattering mechanisms can be well described by this 785 model. In fact, since the X2-Bragg model, like the original 786 X-Bragg, accounts for depolarization in the surface scattering, 787 it is able to properly describe the presence of nonpolarized 788 returns through the $\beta_{1}$ parameter (roughness measure). The 789 fitting of the X2-Bragg to the measured PR indicates that there 790 exists an important contribution of nonpolarized mechanisms 791 ( $\beta_{1}$ values are different from zero, i.e., pure Bragg). Compara792 tively, the X-Bragg model tends to produce an overestimation 793 of the roughness $\left(\beta_{1}\right)$ mainly due to the noise contribution. It 794 has been also recognized that the fully polarimetric data can be 795 exploited to apply an additive NF; otherwise, an overestimation 796 of the PR and thus the roughness for both models is expected.

797 In summary, the study here presented shows that experimen798 tal quad-pol TSX acquisitions over the ocean have intrinsic 799 system/scenario limitations (noise and temporal decorrelation), 800 which should be carefully analyzed to avoid an erroneous inter801 pretation of the polarimetric data. This paper sets the theoretical 802 and analytical foundations to extrapolate the inclusion of such 803 impacts to other physical-based scattering models, evaluating 804 their effectiveness with experimental quad-pol data from cur805 rent state-of-the-art polarimetric SAR sensors.

806

807

808 In the following lines, an extension of the X-Bragg model, 809 referred as X2-Bragg, is presented to introduce the impact of 810 both noise and additional temporal decorrelations, in a two-step 811 procedure.

812 First of all, let us define an equivalent coherency matrix, 813 which accounts for the noise perturbation as (analogous to the 814 study in [19])

$$
\mathbf{T}^{\prime}=\mathbf{T}+\mathbf{N}
$$

815 where $\mathbf{T}$ refers to the expected coherency matrix related to 816 the sea clutter, and the noise coherency matrix is diagonal $817 \mathbf{N}=\sigma_{n}^{2} \mathbf{I}_{3 \times 3}$. For simplicity, it has been assumed that the dif818 ferent polarimetric channels have uncorrelated additive circular 819 complex Gaussian noise with the same power $\sigma_{n}^{2}$.

820 From the previous considerations, and using a general for821 mulation, (8) can be expressed as

$$
\mathbf{T}^{\prime}=\left(\sigma_{1}^{2}+\sigma_{n}^{2}\right) \overline{\mathbf{T}}^{\prime}
$$

822 where the normalized (to $\mathbf{T}_{11}^{\prime}$ ) coherency matrix $\overline{\mathbf{T}}^{\prime}$ is written as

$$
\overline{\mathbf{T}}^{\prime}=\left[\begin{array}{ccc}
1 & \sqrt{\frac{\sigma_{2}^{2}}{\sigma_{1}^{2}} \overbrace{\rho_{\mathrm{SNR}_{1}} \rho_{12}}^{\rho_{T}}} & 0 \\
\sqrt{\frac{\sigma_{2}^{2}}{\sigma_{1}^{2}}} \rho_{\mathrm{SNR}_{1}} \rho_{12}^{*} & \frac{\sigma_{2}^{2}}{\sigma_{1}^{2}} \frac{1+1 / \mathrm{SNR}_{2}}{1+1 / \mathrm{SNR}_{1}} & 0 \\
0 & 0 & \frac{\sigma_{3}^{2}}{\sigma_{1}^{2}} \frac{1+1 / \mathrm{SNR}_{3}}{1+1 / \mathrm{SNR}_{1}}
\end{array}\right]
$$

where $\sigma_{1}^{2}, \sigma_{2}^{2}$, and $\sigma_{3}^{2}$ refer to the signal power of the three 823 polarimetric channels (in the Pauli base); $\mathrm{SNR}_{1}, \mathrm{SNR}_{2}$, and 824 $\mathrm{SNR}_{3}$ are the corresponding $\mathrm{SNR}$, i.e., $\mathrm{SNR}_{i}=\sigma_{i}^{2} / \sigma_{n}^{2} ; \rho_{12} 825$ represents the polarimetric correlation (coherence) coefficient 826 between $\left(S_{\mathrm{HH}}+S_{\mathrm{VV}}\right)$ and $\left(S_{\mathrm{HH}}-S_{\mathrm{VV}}\right)$ channels; and $\rho_{\mathrm{SNR}_{1}} 827$ models the decorrelation effect induced by the presence of 828 thermal noise, i.e.,

$$
\rho_{\mathrm{SNR}_{1}}=\frac{1}{1+1 / \mathrm{SNR}_{1}}
$$

such that the total coherence, based on a multiplicative model, 830 is defined as $\rho_{T}$.

From the original X-Bragg model formulation in (7), the 832 different parameters in (10) can be expressed as

$$
\begin{aligned}
\sigma_{1}^{2} & =m_{s}^{2} C_{1} \\
\sigma_{2}^{2} & =m_{s}^{2} C_{3}\left(1+\operatorname{sinc}\left(4 \beta_{1}\right)\right) \\
\sigma_{3}^{2} & =m_{s}^{2} C_{3}\left(1-\operatorname{sinc}\left(4 \beta_{1}\right)\right) \\
\rho_{12} & =\frac{C_{2} \operatorname{sinc}\left(2 \beta_{1}\right)}{\sqrt{C_{1} C_{3}\left(1+\operatorname{sinc}\left(4 \beta_{1}\right)\right)}} .
\end{aligned}
$$

From the previous relationships, the three polarimetric SNRs 834 can be defined in terms of an equivalent $\mathrm{SNR}_{\mathrm{Eq}}$ as

$$
\begin{aligned}
& \mathrm{SNR}_{1}=C_{1} \mathrm{SNR}_{\mathrm{Eq}} \\
& \mathrm{SNR}_{2}=C_{3}\left(1+\operatorname{sinc}\left(4 \beta_{1}\right)\right) \mathrm{SNR}_{\mathrm{Eq}} \\
& \mathrm{SNR}_{3}=C_{3}\left(1-\operatorname{sinc}\left(4 \beta_{1}\right)\right) \mathrm{SNR}_{\mathrm{Eq}}
\end{aligned}
$$

in a way that, if $\mathrm{SNR}_{1}$ is estimated from the data, the equivalent 836 $\mathrm{SNR}_{\mathrm{Eq}}=m_{s}^{2} / \sigma_{n}^{2}$ can be inverted from the model and used to 837 properly account for the thermal noise impact in the X-Bragg 838 model fitting.

839

In multichannel adaptive array and GMTI theory, the impact 840 of temporal decorrelation is known to cause an increase in 841 the number of eigenvalues (of the clutter-plus-noise covariance 842 matrix) different from the noise floor [3]. The X-Bragg model 843 has been further extended to account for temporal decorrelation, 844 in terms of an additional coherence coefficient $\rho_{\text {temp }}$. It must 845 be noted that this term cannot be directly introduced in matrix 846 (10) since, from the DRA acquisition itself, the temporal decor- 847 relation is between the individual polarimetric channels with 848 some spatial or temporal baseline. This additional term should 849 be considered in the covariance matrix $\mathbf{C}$ formulation. First, a 850 transformation of the normalized coherency matrix $\overline{\mathbf{T}}^{\prime}$ (10) into 851 the covariance matrix is performed as

$$
\overline{\mathbf{C}}^{\prime}=\left\{\mathbf{U}_{3(\mathrm{~L} \rightarrow \mathrm{P})}\right\}^{-1} \overline{\mathbf{T}}^{\prime}\left\{\mathbf{U}_{3(\mathrm{~L} \rightarrow \mathrm{P})}^{H}\right\}^{-1}
$$

where the unitary transformation $(\mathrm{L} \rightarrow \mathrm{P})$ from the lexico- 853 graphic target vector to the Pauli vector is

$$
\mathbf{U}_{3(\mathrm{~L} \rightarrow \mathrm{P})}=\frac{1}{\sqrt{2}}\left[\begin{array}{ccc}
1 & 0 & 1 \\
1 & 0 & -1 \\
0 & \sqrt{2} & 0
\end{array}\right]
$$

Then, the additional coherence coefficient $\rho_{\text {temp }}$ is included 855 as multiplicative term into the off-diagonal terms of $\overline{\mathbf{C}}^{\prime}$ to obtain 856 $\overline{\mathbf{C}}^{\prime \prime}$. The normalized coherency matrix for the X2-Bragg model 857 


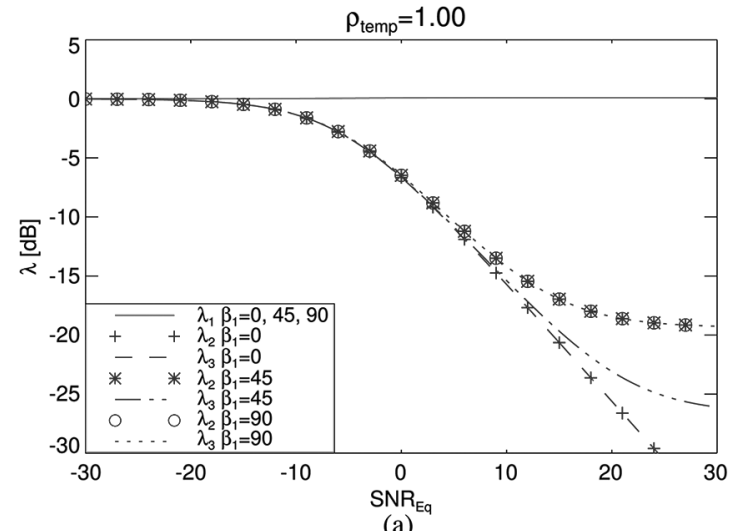

(a)

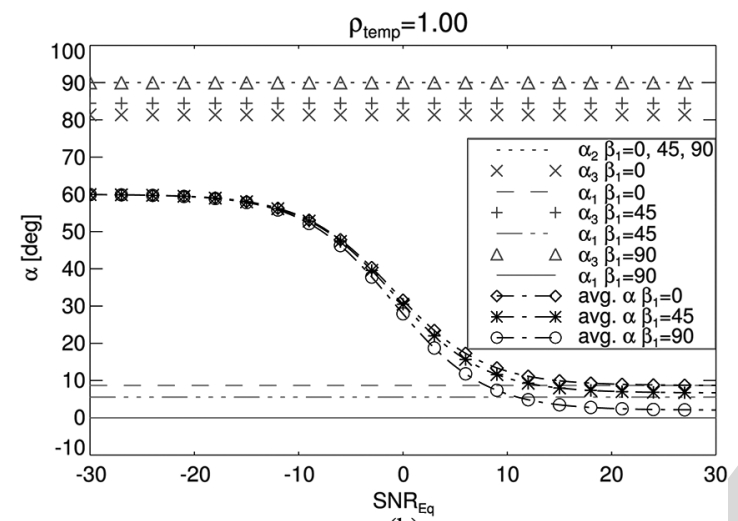

(b)

Fig. 11. Sensitivity w.r.t. $\mathrm{SNR}_{\mathrm{Eq}}$ for the X2-Bragg model considering the noise impact and a temporal decorrelation $\rho_{\text {temp }}=1.0$ for three values of the uniform distribution width $\beta_{1}$; an X-band system $(9.65 \mathrm{GHz})$, an incidence angle $\gamma=24.6^{\circ}$, a sea temperature of $10^{\circ} \mathrm{C}$, and salinity of $35 \%$ are considered. (a) Eigenvalues. (b) Alphas.

$858 \overline{\mathbf{T}}^{\prime \prime}$ can be obtained using a matrix transformation of $\overline{\mathbf{C}}^{\prime \prime}$ by 859 means of $\mathbf{U}_{3(\mathrm{~L} \rightarrow \mathrm{P})}$, inversely analogous to the case in (14).

860

861

862 The sensitivity of the eigenvalues and $\alpha$ parameters as a 863 function of $\mathrm{SNR}_{\mathrm{Eq}}$ for $\rho_{\text {temp }}=1.0$ and $\rho_{\mathrm{temp}}=0.95$ is shown, 864 respectively, in Figs. 11 and 12, exploiting the theoretical 865 coherency matrix formulation $\overline{\mathbf{T}}^{\prime \prime}$ of the X2-Bragg model. It 866 has been assumed an X-band system $(9.65 \mathrm{GHz})$, an incidence 867 angle $\gamma$ of $24.6^{\circ}$ [corresponding to the center of TSX-4 acquisi868 tion (see Table I)], and sea conditions of $10^{\circ} \mathrm{C}$ and $35 \%$ salinity 869 content. In Figs. 11(a) and 12(a), the eigenvalue distribution 870 is represented as a function of $\mathrm{SNR}_{\mathrm{Eq}}$. The three eigenvalues 871 collapse, regardless of $\beta_{1}$ and $\rho_{\text {temp }}$, when reducing $\mathrm{SNR}_{\mathrm{Eq}}$ $872(<-10 \mathrm{~dB})$, being the noise the dominant mechanism.

873 In case of $\beta_{1}=0^{\circ}$ (smooth surface) and with increasing $874 \mathrm{SNR}_{\mathrm{Eq}}$, the two smallest eigenvalues $\lambda_{2}$ (plus symbols) and $\lambda_{3}$ 875 (dashed line) have the same decreasing trend when no temporal 876 decorrelation is included [see Fig. 11(a)]. Their contribution is $87730 \mathrm{~dB}$ below $\lambda_{1}$ for $\mathrm{SNR}_{\mathrm{Eq}}>20 \mathrm{~dB}$, in which case SPM ap878 plies with a single dominant mechanism. This can be observed 879 also from the averaged value $\bar{\alpha}$ [dash-dot line with diamond 880 markers in Fig. 11(b)], which tends to $\alpha_{1}$ (dashed line). As $\beta_{1}$

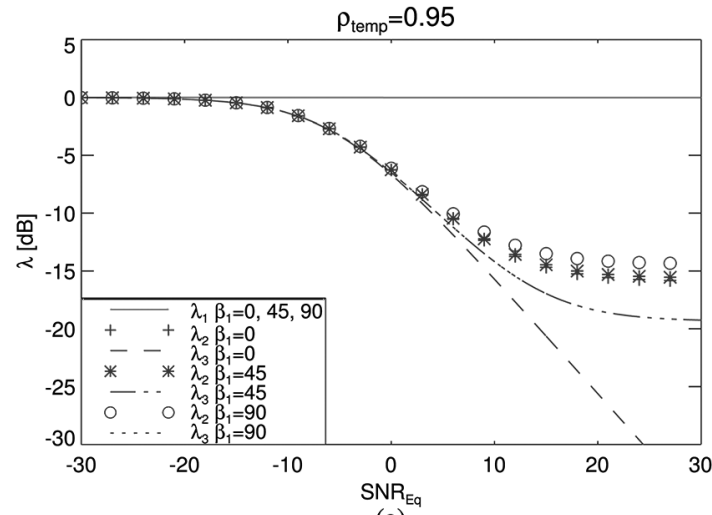

(a)

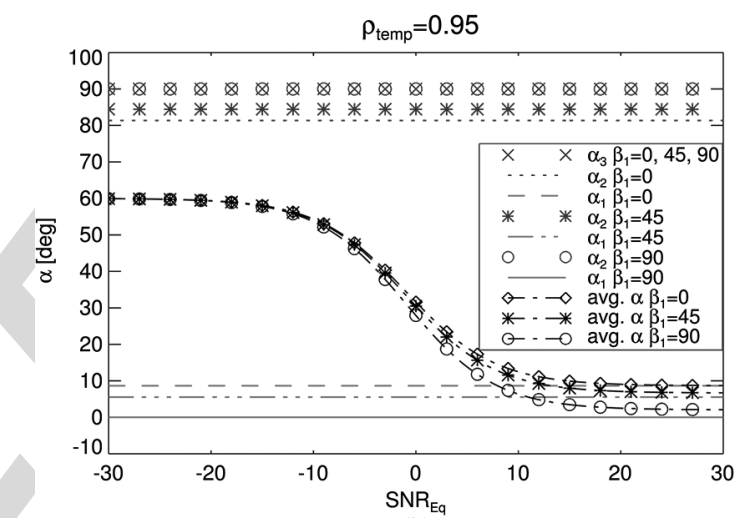

(b)

Fig. 12. Sensitivity w.r.t. $\mathrm{SNR}_{\mathrm{Eq}}$ for the X2-Bragg model considering the noise impact and a temporal decorrelation $\rho_{\text {temp }}=0.95$ for three values of the uniform distribution width $\beta_{1}$; an X-band system $(9.65 \mathrm{GHz})$, an incidence angle $\gamma=24.6^{\circ}$, a sea temperature of $10^{\circ} \mathrm{C}$, and salinity of $35 \%$ are considered. (a) Eigenvalues. (b) Alphas.

(roughness) increases, the cross-polar power increases; hence, 881 more than a single dominant mechanism exist. For $\beta_{1}=45^{\circ} 882$ and $\mathrm{SNR}_{\mathrm{Eq}}>10 \mathrm{~dB}$, the two smallest eigenvalues (asterisk 883 symbols and dash-dot-dot line) start to diverge. In the limit 884 $\beta_{1}=90^{\circ}$ and with no additional temporal decorrelation, $\lambda_{2} 885$ (circle markers) and $\lambda_{3}$ (dotted line) have the same trend as a 886 function of the effective SNR, since polarimetric coherence $\rho_{12} 887$ decreases to 0 . Considering a temporal decorrelation of the sea 888 $\rho_{\text {temp }}=0.95$, a general increase of the second eigenvalue can 889 be recognized (for $\mathrm{SNR}_{\mathrm{Eq}}>10 \mathrm{~dB}$ ), comparing Figs. 11(a) and 890 12(a), particularly for $\beta_{1}=0^{\circ}$. In case of $\lambda_{3}$, this behavior is 891 also observed, but for $\beta_{1} \geq 45^{\circ}$.

Contrary to the eigenvalues, the individual $\alpha$ parameters 893 are almost unaffected by the presence of noise, as shown in 894 Figs. 11(b) and 12(b). Polarimetric decomposition considers 895 the mean $\bar{\alpha}$, based on proper weighting of the individual $\alpha 896$ terms through the eigenvalues' probabilities [14]. As shown in 897 Figs. 11(b) and 12(b), the average $\bar{\alpha}$ presents a large variation 898 as a function of $\mathrm{SNR}_{\mathrm{Eq}}$ for different values of $\beta_{1}$. In the limit, 899 $\bar{\alpha}$ tends to $60^{\circ}$, such that the scattering mechanism lies in the 900 extreme edge of region 2 of the Cloude-Pottier $H / \bar{\alpha}$, i.e., 901 random noise, that is, no polarization dependence. Temporal 902 decorrelation exchanges the behavior of the individual $\alpha_{2}$ and 903 $\alpha_{3}$ parameters, comparing Figs. 11(b) and 12(b), but does not 904 affect the averaged values: when no temporal decorrelation 905 


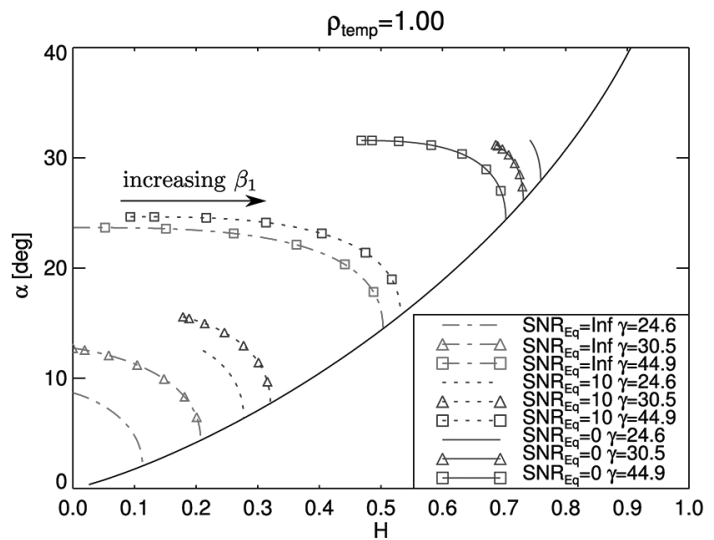

(a)

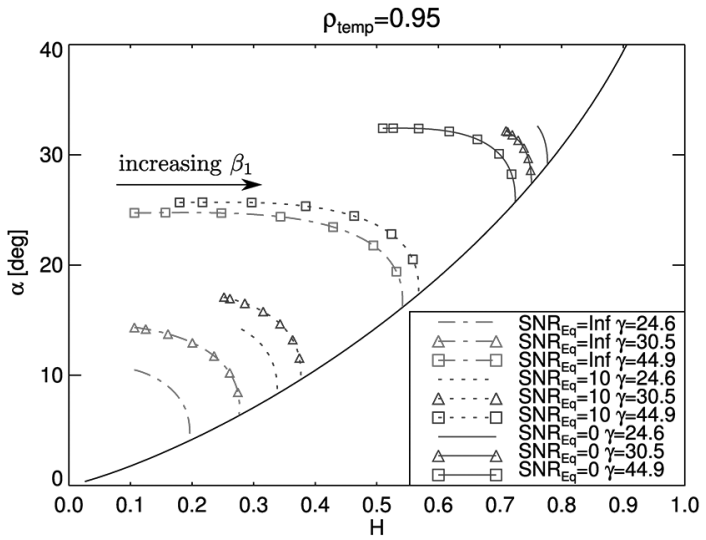

(b)

Fig. 13. $H / \bar{\alpha}$ plane for the X2-Bragg model at three incidence angles $\left(24.6^{\circ}, 33.8^{\circ}\right.$, and $\left.44.9^{\circ}\right)$ and for three $\mathrm{SNR}_{\mathrm{Eq}}$ conditions $(\infty, 10 \mathrm{~dB}$, and $0 \mathrm{~dB})$ : (a) $\rho_{\text {temp }}=1.0$ and (b) $\rho_{\text {temp }}=0.95$ (sea water with salinity content of $35 \%$ and temperature of $10^{\circ} \mathrm{C}$ ).

906 is considered $\left(\rho_{\text {temp }}=1\right), \alpha_{2}$ collapses to $90^{\circ}$ regardless of $907 \beta_{1}$, and $\alpha_{3}$ sweeps the values from $80^{\circ}$ to $90^{\circ}$, whereas for $908 \rho_{\text {temp }}=0.95, \alpha_{3}$ is $90^{\circ}$ for the different roughness parameters. 909 Therefore, temporal decorrelation is not impairing the values 910 of $\alpha$ parameters, but it is modulating the contribution of the dif911 ferent mechanisms, i.e., for $\rho_{\text {temp }}=0.95$, the cross-polar com912 ponent (related to $\alpha=90$ independently from $\beta_{1}$ ) is no longer 913 the second dominant scattering mechanism (as for $\rho_{\text {temp }}=1$ ). 914 The impact of both thermal noise and temporal decorre915 lation in the X2-Bragg model is also analyzed in the $H / \bar{\alpha}$ 916 plane, as represented in Fig. 13(a) and (b) for $\rho_{\text {temp }}=1$ and $917 \rho_{\text {temp }}=0.95$, respectively. Different incidence angles, corre918 sponding to the center of data takes TSX-4 $\left(24.6^{\circ}\right)$, TSX-7 $919\left(30.5^{\circ}\right)$, and TSX-17 $\left(44.9^{\circ}\right)$, are considered. The noise impact 920 is translated into a progressive increase of both $\bar{\alpha}$ and $H$ as $921 \mathrm{SNR}_{\mathrm{Eq}}$ decreases. Moreover, the variation of the $H / \bar{\alpha}$ pairs as a 922 function of $\beta_{1}$ is reduced when the noise contribution increases. 923 Temporal decorrelation produces similar effects, but its impact 924 reduces as long as the entropy increases, clearly visible for the 925 region with $H>0.6$ when comparing Fig. 13(a) and (b).

926 A particular case in the $H / \bar{\alpha}$ plane is recognized for $927 \mathrm{SNR}_{\mathrm{Eq}}=0 \mathrm{~dB}$, where the variation as a function of the in928 cidence angle is reversed, i.e., the near range presents higher 929 entropy values than the far range: as long as the noise con930 tribution increases, the divergence between the two smallest 931 eigenvalues $\left(\lambda_{2}\right.$ and $\left.\lambda_{3}\right)$ reduces (increasing the entropy), and 932 this effect is more severe for the near range. This relative sepa933 ration is also a function of the roughness $\left(\beta_{1}\right)$, which, for noisy 934 near range scenarios, is quite flat. Such a response justifies 935 the reduction on $H$ as a function of $\beta_{1}$ for increasing noise, 936 particularly at near range.

\section{ACKNOWLEDGMENT}

938 The authors would like to thank the German Aerospace 939 Center (DLR) and, in particular, S. Baumgartner for the TSX 940 quad-pol data provided in the frame of the DRA proposal 941 MTH1971 and the FP7-SPACE Project NEREIDS Ref. 263468 942 for the TSX dual-pol data. The authors would also like to thank 943 A. Gómez for her helpful suggestions and comments.

\section{REFERENCES}

[1] S. Hasselmann, C. Brüning, K. Hasselmann, and P. Heimbach, "An im- 945 proved algorithm for the retrieval of ocean wave spectra from synthetic 946 aperture radar image spectra," J. Geophys. Res., Oceans, vol. 101, no. C7, 947 pp. 16615-16629, Jul. 1996.

\section{8}

[2] R. Goldstein and H. Zebker, "Interferometric radar measurement of ocean 949 surface currents," Nature, vol. 328, no. 6132, pp. 707-709, Aug. 1987.950

[3] E. Makhoul, A. Broquetas, J. Ruiz-Rodon, Y. Zhan, and F. Ceba, 951 "A performance evaluation of SAR-GMTI missions for maritime applica- 952 tions," IEEE Trans. Geosci. Remote Sens., vol. 53, no. 5, pp. 2496-2509, 953 May 2015.

[4] M. Gabele et al., "Fore and aft channel reconstruction in the TerraSAR-X 955 dual receive antenna mode," IEEE Trans. Geosci. Remote Sens., vol. 48, 956 no. 2, pp. 795-806, Feb. 2010.

[5] I. Hajnsek, E. Pottier, and S. Cloude, "Inversion of surface parameters 958 from polarimetric SAR," IEEE Trans. Geosci. Remote Sens., vol. 41, 959 no. 4, pp. 727-744, Apr. 2003.

[6] X.-M. Li and S. Lehner, "Algorithm for sea surface wind retrieval from 961 TerraSAR-X and TanDEM-X data," IEEE Trans. Geosci. Remote Sens., 962 vol. 52, no. 5, pp. 2928-2939, May 2014.

[7] T. Fritz and M. Eineder, "TerraSAR-X: Ground segment basic product 964 specification document," German Aerosp. Center (DLR), Köln, Germany, 965 Tech. Rep. TX-GS-DD-3302, Oct. 2013.

[8] K. D. Ward, R. J. Tough, and S. Watts, Sea Clutter: Scattering, the K 967 Distribution and Radar Performance. Stevenage, U.K.: The Insti. Eng. 968 Technol., 2006.

[9] S. Anfinsen and T. Eltoft, "Application of the matrix-variate Mellin trans- 970 form to analysis of polarimetric radar images," IEEE Trans. Geosci. 971 Remote Sens., vol. 49, no. 6, pp. 2281-2295, Jun. 2011.

[10] S. Skrunes, C. Brekke, T. Eltoft, and V. Kudryavtsev, "Comparing 973 near-coincident C- and X-band SAR acquisitions of marine oil spills," 974 IEEE Trans. Geosci. Remote Sens., vol. 53, no. 4, pp. 1958-1975, 975 Apr. 2015.

[11] E. Jakeman and P. N. Pusey, "A model for non-Rayleigh sea echo," IEEE 977 Trans. Antennas Propag., vol. AP-24, no. 6, pp. 806-814, Nov. 1976.978

[12] K. Ouchi and R. Cordey, "Statistical analysis of azimuth streaks observed 979 in digitally processed CASSIE imagery of the sea surface," IEEE Trans. 980 Geosci. Remote Sens., vol. 29, no. 5, pp. 727-735, Sep. 1991.

[13] D. Trizna, "Statistics of low grazing angle radar sea scatter for moder- 982 ate and fully developed ocean waves," IEEE Trans. Antennas Propag., 983 vol. 39, no. 12, pp. 1681-1690, Dec. 1991.

[14] S. Cloude and E. Pottier, "An entropy based classification scheme for land 985 applications of polarimetric SAR,” IEEE Trans. Geosci. Remote Sens., 986 vol. 35, no. 1, pp. 68-78, Jan. 1997.

[15] V. Kudryavtsev, D. Hauser, G. Caudal, and B. Chapron, "A semiempirical 988 model of the normalized radar cross-section of the sea surface 1. Back- 989 ground model," J. Geophys. Res., Oceans, vol. 108, no. C3, pp. FET 2-1- 990 FET 2-24, Mar. 2003.

[16] J. Morris, S. Anderson, and S. Cloude, "A study of the X-band entropy 992 of breaking ocean waves," in Proc. IEEE IGARSS, Jul. 2003, vol. 2, 993 pp. 711-713.

[17] D. A. Gagliardini and P. C. Colón, "Ocean feature detection using mi- 995 crowave backscatter and sun glint observations," Gayana (Concepción), 996 vol. 68 , no. 2 , pp. 180-185, 2004. 
998 [18] AIRBUS, "Radiometric calibration of TerraSAR-X data: Beta naught and 999 sigma naught coefficient calculation," AIRBUS Defence Space, Munich, 1000 Germany, Tech. Rep. TSXX-ITD-TN-0049, Mar. 2014.

1001 [19] C. López-Martínez, A. Alonso-González, and X. Fabregas, "Perturbation 1002 analysis of eigenvector-based target decomposition theorems in radar po1003 larimetry," IEEE Trans. Geosci. Remote Sens., vol. 52, no. 4, pp. 20811004 2095, Apr. 2014.

1005 [20] V. Gregers-Hansen and R. Mital, "An improved empirical model for radar 1006 sea clutter reflectivity,” IEEE Trans. Aerosp. Electron. Syst., vol. 48, no. 4, 1007 pp. 3512-3524, Oct. 2012.

1008 [21] F. E. Nathanson, J. P. Reilly, and M. N. Cohen, Radar Design Principles: 1009 Signal Processing and the Environment, 2nd ed. New York, NY, USA: 1010 McGraw-Hill, 1990

1011 [22] M. Menon, "Estimation of the shape and scale parameters of the Weibull 1012 distribution," Technometrics, vol. 5, no. 2, pp. 175-182, May 1963.

1013 [23] D. Iskander and A. Zoubir, "Estimation of the parameters of the 1014 K-distribution using higher order and fractional moments [radar clut1015 ter]," IEEE Trans. Aerosp. Electron. Syst., vol. 35, no. 4, pp. 1453-1457, 1016 Oct. 1999.

1017 [24] D. Blacknell and R. J. A. Tough, "Parameter estimation for the 1018 K-distribution based on [z $\log (\mathrm{z})]$," Proc. Inst. Elect. Eng.-Radar Sonar 1019 Navig., vol. 148, no. 6, pp. 309-312, Dec. 2001.

1020 [25] R. J. A. Tough, D. Blacknell, and S. Quegan, "A statistical description 1021 of polarimetric and interferometric synthetic aperture radar data," Proc. 1022 R. Soc. Lond. A, Math. Phys. Sci., vol. 449, no. 1937, pp. 567-589, 1023 Jun. 1995

1024 [26] D. Schuler and J. Lee, "Mapping ocean surface features using bio1025 genic slick-fields and SAR polarimetric decomposition techniques," Proc. 1026 Inst. Elect. Eng._Radar Sonar Navig., vol. 153, no. 3, pp. 260-270, 1027 Jun. 2006.

1028 [27] M. Migliaccio, A. Gambardella, and M. Tranfaglia, "SAR polarimetry 1029 to observe oil spills," IEEE Trans. Geosci. Remote Sens., vol. 45, no. 2, 1030 pp. 506-511, Feb. 2007.

1031 [28] A. Voronovich and V. Zavorotny, "Theoretical model for scattering of 1032 radar signals in $\mathrm{Ku}$-and $\mathrm{C}$-bands from a rough sea surface with breaking 1033 waves," Waves Random Media, vol. 11, no. 3, pp. 247-269, 2001.

1034 [29] A. A. Mouche, D. Hauser, and V. Kudryavtsev, "Radar scattering of the 1035 ocean surface and sea-roughness properties: A combined analysis from 1036 dual-polarizations airborne radar observations and models in C band," 1037 J. Geophys. Res., Oceans, vol. 111, no. C9, Sep. 2006, Art. ID. C09004.

1038 [30] D. Schuler, J.-S. Lee, and K. Hoppel, "Polarimetric SAR image signatures 1039 of the ocean and gulf stream features," IEEE Trans. Geosci. Remote Sens., 1040 vol. 31, no. 6, pp. 1210-1221, Nov. 1993.

1041 [31] L. Klein and C. Swift, "An improved model for the dielectric constant 1042 of sea water at microwave frequencies," IEEE Trans. Antennas Propag., 1043 vol. AP-25, no. 1, pp. 104-111, Jan. 1977.

1044 [32] R. Romeiser and D. Thompson, "Numerical study on the along-track 1045 interferometric radar imaging mechanism of oceanic surface currents," 1046 IEEE Trans. Geosci. Remote Sens., vol. 38, no. 1, pp. 446-458, Jan. 2000.

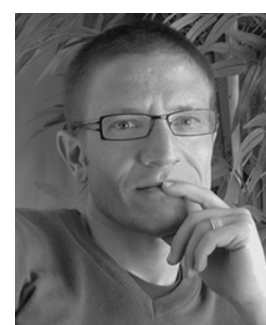

Carlos López-Martínez (S'97-M'04-SM'11) re- 1069 ceived the M.Sc. degree in electrical engineering 1070 and the Ph.D. degree from Universitat Politècnica 1071 de Catalunya (UPC), Barcelona, Spain, in 1999 and 1072 2003, respectively.

1073

From October 2000 to March 2002, he was 1074 with the Frequency and Radar Systems Depart- 1075 ment, HR, German Aerospace Center (DLR), 1076 Oberpfaffenhofen, Germany. From June 2003 to De- 1077 cember 2005, he was with the Image and Remote 1078 Sensing Group SAPHIR Team of the Institute of 1079 Electronics and Telecommunications of Rennes (IETR CNRS UMR 6164), 1080 Rennes, France. In January 2006, he joined UPC, Barcelona, as a Ramón 1081 y Cajal Researcher. He is currently an Associate Professor in the area of 1082 remote sensing and microwave technology with UPC. He has authored or 1083 coauthored over 100 articles in journals, books, and conference proceedings 1084 in the radar remote sensing and image analysis literature. His research interests 1085 include SAR and multidimensional SAR, radar polarimetry, physical parameter 1086 inversion, digital signal processing, estimation theory, and harmonic analysis. 1087

Dr. López-Martínez is an Associate Editor of the IEEE JOURnAL OF SE- 1088 Lected Topics in Applied Earth ObSERvations ANd Remote SEns- 1089 ING. He served as a Guest Editor of the EURASIP Journal on Advances in 1090 Signal Processing. He has organized different invited sessions in international 1091 conferences on radar and SAR polarimetry. He has presented advanced courses 1092 and seminars on radar polarimetry to a wide range of organizations and events. 1093 He was a recipient of the Student Prize Paper Award at the EUSAR 20021094 Conference and coauthored the paper awarded with the First Place Student 1095 Paper Award at the EUSAR 2012 Conference. He has been also a recipient of 1096 the IEEE Geoscience and Remote Sensing Society 2013 GOLD Early Career 1097 Award.

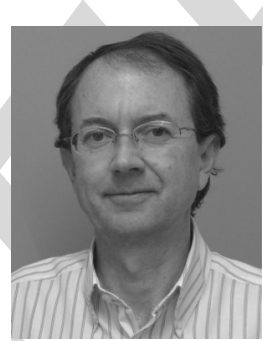

Antoni Broquetas (S'84-M'90) was born in 1099 Barcelona, Spain, in 1959. He received the Ingeniero 1100 degree in telecommunications engineering and the 1101 Doctor Ingeniero degree in telecommunications en- 1102 gineering (for his work on microwave tomography) 1103 from Universitat Politècnica de Catalunya (UPC), 1104 Barcelona, in 1985 and 1989, respectively. 1105

In 1986, he was a Research Assistant with 1106 Portsmouth Polytechnic, Portsmouth, U.K., involved 1107 in propagation studies. In 1987, he joined the Depart- 1108 ment of Signal Theory and Communications (TSC), 1109

UPC, where he was the Subdirector of Research of the Institute of Geomatics 1110 from 1998 to 2002 and the Director of TSC from 2003 to 2006. Since 1111 1999, he has been a Full Professor with UPC, involved in research on radar 1112 imaging and remote sensing. He has authored or coauthored over 150 papers 1113 on microwave tomography, radar, ISAR and SAR systems, and SAR processing 1114 and interferometry.

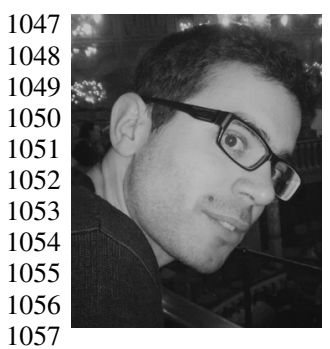

Eduardo Makhoul (S'09-M'15) was born in Homs, Siria, in 1985. He received the M.Sc. degree in electrical engineering and the Ph.D. degree (cum laude) from Universitat Politècnica de Catalunya (UPC), Barcelona, Spain, in 2009 and 2015, respectively.

In 2008 , he worked on his master thesis in the frame of high-resolution wide-swath synthetic aperture radar (SAR) systems at the German Aerospace Center (DLR). In 2009, he joined the Remote Sensing Laboratory, UPC, researching on aspects of ground moving target indication (GMTI) for future 1058 spaceborne SAR systems. From 2009 to 2011, he worked on the radiomet1059 ric budget analysis of the Spanish mission PAZ. From 2010 to 2013, he 1060 worked on the analysis and study of SAR-GMTI systems and techniques for 1061 future European missions in the frame of NEWA and SIMTISYS projects. In 10622013 (April-August), he was a Visiting Researcher with DLR, working on 1063 SAR-GMTI processing of airborne and spaceborne data over maritime scenar1064 ios. Since August 2015, he has been with IsardSAT, an experienced Earth obser1065 vation research company in Barcelona, involved in the design, calibration, and 1066 maintenance of civil remote sensing instruments. His main research interests 1067 include SAR, GMTI, radar system design, mission performance, calibration, 1068 array signal processing, sea/ocean surveillance, and polarimetry.

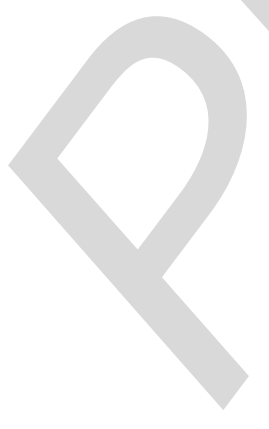




\section{AUTHOR QUERIES}

\section{AUTHOR PLEASE ANSWER ALL QUERIES}

Please be aware that authors are required to pay overlength page charges (\$200 per page) if the paper is longer than 6 pages. If you cannot pay any or all of these charges please let us know.

This pdf contains 2 proofs. The first half is the version that will appear on Xplore. The second half is the version that will appear in print. If you have any figures to print in color, they will be in color in both proofs.

The "Open Access" option for your paper expires when the paper is published on Xplore in an issue with page numbers. Papers in "Early Access" may be changed to "Open Access."

AQ1 = Please check if changes made in the affiliation of author E. Makhoul are correct. Otherwise, please make the necessary changes.

AQ2 = Please provide the expanded form of "DFO."

END OF ALL QUERIES 